\title{
Symmetry-based indicators for topological Bogoliubov-de Gennes Hamiltonians
}

Geier, Max ; Brouwer, Piet W ; Trifunovic, Luka

\begin{abstract}
We develop a systematic approach for constructing symmetry-based indicators of a topological classification for superconducting systems. The topological invariants constructed in this work form a complete set of symmetry-based indicators that can be computed from knowledge of the Bogoliubovde Gennes Hamiltonian on high-symmetry points in the Brillouin zone. After excluding topological invariants corresponding to the phases without boundary signatures, we arrive at a natural generalization of symmetry-based indicators [H. C. Po, A. Vishwanath, and H. Watanabe, Nat. Commun. 8, 50 (2017)] to Hamiltonians of Bogoliubov-de Gennes type.
\end{abstract}

DOI: https://doi.org/10.1103/physrevb.101.245128

Posted at the Zurich Open Repository and Archive, University of Zurich ZORA URL: https://doi.org/10.5167/uzh-188893

Journal Article

Published Version

Originally published at:

Geier, Max; Brouwer, Piet W; Trifunovic, Luka (2020). Symmetry-based indicators for topological Bogoliubov-de Gennes Hamiltonians. Physical review B, 101(24):245128.

DOI: https://doi.org/10.1103/physrevb.101.245128 


\title{
Symmetry-based indicators for topological Bogoliubov-de Gennes Hamiltonians
}

\author{
Max Geier $\odot,{ }^{1}$ Piet W. Brouwer, ${ }^{1}$ and Luka Trifunovic ${ }^{1,2,3}$ \\ ${ }^{1}$ Dahlem Center for Complex Quantum Systems and Physics Department, Freie Universität Berlin, Arnimallee 14, 14195 Berlin, Germany \\ ${ }^{2}$ RIKEN Center for Emergent Matter Science, Wako, Saitama 351-0198, Japan \\ ${ }^{3}$ Department of Physics, University of Zurich, Winterthurerstrasse 190, 8057 Zurich, Switzerland
}

(Received 18 November 2019; accepted 28 April 2020; published 8 June 2020)

\begin{abstract}
We develop a systematic approach for constructing symmetry-based indicators of a topological classification for superconducting systems. The topological invariants constructed in this work form a complete set of symmetry-based indicators that can be computed from knowledge of the Bogoliubov-de Gennes Hamiltonian on high-symmetry points in the Brillouin zone. After excluding topological invariants corresponding to the phases without boundary signatures, we arrive at a natural generalization of symmetry-based indicators [H. C. Po, A. Vishwanath, and H. Watanabe, Nat. Commun. 8, 50 (2017)] to Hamiltonians of Bogoliubov-de Gennes type.
\end{abstract}

DOI: 10.1103/PhysRevB.101.245128

\section{INTRODUCTION}

Although topological phases of matter have been known for four decades now, starting with the discovery of the quantized Hall effect [1], the study of topological phases and phase transitions became central to quantum condensed matter physics only in the early 2000s, after the theoretical proposals for topological superconducting phases $[2,3]$ and the quantum spin Hall effect $[4,5]$ and the subsequent experimental observation of these phases of matter [6,7]. These theoretical and experimental developments paved the way for the complete classification of all possible topological phases of single-particle systems, protected by either time-reversal, particle-hole, or sublattice symmetries, the so-called "tenfoldway classification" [8], which, reflecting its periodicity as a function of dimensionality and honoring its fundamental importance to the field, was coined "periodic table of topological phases" $[9,10]$.

In addition to the presence or absence of the fundamental nonspatial symmetries that define the tenfold-way classes, real materials have crystalline symmetries. The combination of topology and crystalline symmetries leads to an exceedingly rich set of "topological crystalline phases" [11]. Unlike noncrystalline tenfold-way topological phases, for which a nontrivial topology of the bulk is always associated with a unique anomalous boundary signature, topological crystalline phases may come with a variety of possible boundary signatures. These include the protected existence of anomalous boundary states on all boundaries, a "first-order" topological phase, for which the crystalline symmetry is not essential for the protection of the nontrivial topology, the appearance of higher-order boundary states on hinges or corners of a crystal [12-26], or even the complete absence of protected boundary states. The latter scenario applies to "atomic-limit phases," in which the electronic states can be continuously deformed to a collection of localized orbitals, while preserving all relevant symmetries as discussed by Po et al. [27] and Bradlyn et al. [28]. In such cases, the presence of the crystalline symmetry may form an "obstruction" that prevents from different arrangements of localized orbitals to be continuously connected to each other, ${ }^{1}$ allowing for the existence of multiple topologically distinct atomic-limit phases [29-35].

Although the classification of topological crystalline phases can be considered largely under control [36-43] (for initial partial classifications results, see Refs. [44-56]), the explicit computation of topological invariants for a given band structure is often computationally expensive. Explicit expressions for the invariants need not always be readily available since a full classification does not always come with explicit expressions for topological invariants. A practical, but partial, solution to this problem is the use of a set of easy-tocompute "symmetry-based indicators" which, when nonzero, are a sufficient indicator of a nontrivial topology of the bulk band structure [27]. The Fu-Kane criterion, which links the existence of a strong ${ }^{2}$ topological insulator phase in an inversion-symmetric crystal to the parity of the number of occupied bands with odd-inversion parity at the high-symmetry momenta, is an example of such a symmetry-based indicator [57]. For normal-state insulating phases, symmetry-based indicators were constructed for the complete set of point-group symmetries in two and three dimensions [27,28,58,59], taking into account the order of the boundary states [24,60]. Like the classifying groups of topological phases, symmetry-based indicators of topological phases have a group structure, the group operation being the direct sum " $\oplus$ " of representative Hamiltonians. ${ }^{3}$

\footnotetext{
${ }^{1}$ Although atomic-limit phases have no protected boundary states, they may have protected fractional charges at ends or corners (see, e.g., Refs. [31-33]).

${ }^{2}$ In this work, the term "strong topological phase" is reserved for a phase that remains topologically nontrivial after the translational symmetry is broken. Topological crystalline phases that do not rely on the crystalline symmetry for their protection will be referred to as "first order," corresponding to the dimension of their boundary signature.

${ }^{3}$ Although Refs. [27,28] have equivalent definitions of "atomiclimit phases," Bradlyn et al. [28] consider band labels that lack the
} 
The general strategy underlying the construction of symmetry-based indicators for a Hamiltonian $h(\mathbf{k})$ is to replace the topological classification of the matrix-valued function $h(\mathbf{k})$ by the simpler problem of the topological characterization of the Hermitian matrices $h\left(\mathbf{k}_{\mathrm{s}}\right)$ at a selected set of "high-symmetry" points $\left\{\mathbf{k}_{\mathrm{s}}\right\}$ in the Brillouin zone [27]. The topological characterization of the Hermitian matrices $h\left(\mathbf{k}_{\mathrm{s}}\right)$ may be considered a set of "topological band labels," the calculation of which is considerably easier to obtain than the topological classification of the full functions $h(\mathbf{k})$. The group SI of symmetry-based indicators then follows by "dividing out" all combinations of topological band labels that correspond to atomic phases and imposing a set of compatibility constraints derived from the topological classification of the matrices $h(\mathbf{k})$ at lower-symmetry points in the Brillouin zone $[27,28]$. This procedure ensures that only topological phases with a nontrivial boundary signature have nontrivial symmetry-based indicators.

Despite their enormous computational advantage [28], symmetry-based indicators are not guaranteed to give complete classification information: there exist topologically nontrivial phases with protected anomalous boundary signatures, but trivial symmetry-based indicators. An example is the quantized Hall effect in the absence of any crystalline symmetries, for which no symmetry-based indicators exist (i.e., the group SI is trivial), in spite of the existence of topological phases with nonzero Chern number.

In this paper, we extend the construction of symmetrybased indicators to superconductors, which, on the mean-field level, are described by Hamiltonians $H(\mathbf{k})$ of Bogoliubovde Gennes (BdG) type [61,62]. We explicitly construct the topological band labels and the symmetry-based indicators for selected point groups using the complete topological classification of the $\mathrm{BdG}$ Hamiltonian $H\left(\mathbf{k}_{\mathrm{s}}\right)$ at high-symmetry momenta $\mathbf{k}_{\mathrm{s}}$ and compare the symmetry-based indicators with a full classification of topological phases with nontrivial boundary signature. Such a comparison gives information to what extent the symmetry-based indicators can be used as a proxy for a complete classification. Depending on the crystalline symmetries considered, we find that certain aspects of the wealth of boundary signatures available to superconducting phases (Majorana modes at surfaces or hinges, zeroenergy Majorana bound states at corners) are reflected in the symmetry-based indicators, but not all.

full group structure: one can perform addition of the band labels, but not their subtraction. Subsequent careful comparison revealed that some phases without protected boundary states are topologically equivalent to a "difference" of atomic-limit phases, i.e., they can only be deformed to an atomic-limit phase after addition of topologically trivial bands [see H. C. Po, H. Watanabe, and A. Vishwanath, Phys. Rev. Lett. 121, 126402 (2018)]. Such phases were called "fragile topological insulators." They are trivial in the classifying scheme of Po et al. [27], while Bradlyn et al. [28] label them as nontrivial. The extension of the definition of atomic-limit insulators to superconductors discussed in this work allows one to define "fragile topological superconductors" in an analogous way, although this analogy is not being pursued here.
Recently, a number of papers appeared in the literature that also consider the construction of symmetry-based indicators for superconducting phases. Based on a general analysis of the principles underlying the construction of symmetry-based indicators, Ono and Watanabe [63] arrive at the conclusion that the sets of symmetry-based indicators that describe superconducting phases and the underlying normal-state phases are essentially the same. Our construction of symmetry-based indicators, which is based on the full classification of "zerodimensional" Hamiltonians at high-symmetry momenta [64], shows that this statement needs to be corrected to the extent that we successfully put to use a topological band label based on the Pfaffian of a BdG-type Hamiltonian $H\left(\mathbf{k}_{\mathrm{s}}\right)$ at high-symmetry momenta $\mathbf{k}_{\mathrm{s}}$, which has no counterpart in the normal state. A different approach to the problem of symmetry-based indicators in superconductors was taken in a later paper by these authors, together with Yanase [65], as well as by Skurativska, Neupert, and Fischer [66], who consider the "weak-pairing limit" (superconducting order parameter $\Delta$ much smaller than energy scales typical for the normal-state band structure) and derive a classification of superconducting phases that is based on the topological classification of the normal state and the symmetry of the superconducting order parameter. An approach that uses the normal-state Hamiltonian as its sole input has the practical advantage that the symmetry-based indicators can be calculated from the vast body of band-structure knowledge available for normal phases. Furthermore, we show that there exists a consistent definition of an "atomic-limit superconductor" as an "array" of zero-dimensional superconductors [39]. Our definition is consistent with the definition in the published version of Skurativska et al. [65]. In the weak-pairing limit, the symmetrybased indicators derived here can be expressed in terms of the normal part of the Hamiltonian only, so that in that limit our approach offers the same computational advantages as the approaches that rely on the weak-pairing limit at the outset. Very recently, a paper by Ono, Po, and Watanabe appeared [67], which bases its symmetry indicators on the full BdG Hamiltonian, be it without the Pfaffian band labels, and has a definition of an atomic limit that is consistent with ours. A recent paper by Shiozaki also reports the construction of symmetry-based indicators for Hamiltonians of BdG type and which has results very similar to ours [68].

The remaining part of this paper is organized as follows: In Sec. II we discuss the symmetry of the superconducting order parameter and the set of data that needs to be specified in order to define a "topological class" for a crystalline superconductor. In Sec. III we discuss the classification and topological invariants of zero-dimensional Hamiltonians. This discussion is the cornerstone for defining topological invariants at high-symmetry momenta in Brillouin zone. We present our main result, a method to construct and calculate symmetry-based indicators for superconductors, in Sec. IV. Sections V-VII contain examples for various crystalline symmetries compatible with a square or cubic lattice structure, for which we present a detailed calculation of symmetry-based indicators and relate this to higher-order boundary phenomenology. We conclude in Sec. VIII. The Appendices contain additional examples as well as fully worked-out classifications of anomalous boundary states for 
crystalline symmetry groups not readily available in the literature.

\section{SUPERCONDUCTORS WITH CRYSTALLINE SYMMETRIES}

The mean-field theory of superconductors uses an effective noninteracting description with a Hamiltonian of the Bogoliubov-de Gennes (BdG) form [61,62]

$$
H(\mathbf{k})=\left(\begin{array}{cc}
h(\mathbf{k}) & \Delta(\mathbf{k}) \\
\Delta^{\dagger}(\mathbf{k}) & -h^{*}(-\mathbf{k})
\end{array}\right),
$$

where the normal-state Hamiltonian $h(\mathbf{k})$ is Hermitian and the superconducting order parameter $\Delta(\mathbf{k})=-\Delta(-\mathbf{k})^{\mathrm{T}}$ is antisymmetric. The $2 \times 2$ block structure describes particle and hole degrees of freedom. The special choice of the blocks in Eq. (1) is equivalent to imposing that $H(\mathbf{k})$ is antisymmetric under particle-hole conjugation $\mathcal{P}=\tau_{1} K$,

$$
H(\mathbf{k})=-\tau_{1} H(-\mathbf{k})^{*} \tau_{1},
$$

where $\tau_{1}$ is a Pauli matrix acting within particle-hole space and $K$ is complex conjugation. (Note that $\mathbf{k} \rightarrow-\mathbf{k}$ under complex conjugation.) In addition to the BdG structure, we assume that the system obeys translation invariance, which is what allows us to use the Fourier language in Eqs. (1) and (2) in the first place, and that it has additional symmetries described by the point group $G$. We restrict ourselves to symmorphic symmetries, for which the unit cell can be chosen in such a way that it is left invariant under $G$. For the initial discussion we focus on systems without time-reversal symmetry or other antiunitary symmetries.

Requiring that the normal part $h(\mathbf{k})$ be symmetric under $G$ implies that there exists a projective representation $u(g)$ for $g \in G$ such that

$$
h(\mathbf{k})=u(g) h(g \mathbf{k}) u^{\dagger}(g) .
$$

Note that the unitary matrix $u(g)$ does not depend on $\mathbf{k}$, as the symmetry group $G$ acts within the unit cell. The representation $u(g)$ is projective because the transformation rule (3) determines $u(g)$ up to a phase factor only. In general, a consistent choice of these phase factors is possible up to a sign only, the sign ambiguities being captured by the "factor system"

$$
\left\{z_{g, h}=u(g h)^{-1} u(g) u(h)= \pm 1 \text { for } g, h \in G\right\}
$$

of the projective representation. Two realizations $u_{1}(g)$ and $u_{2}(g)$ that have the same factor system may still differ by a one-dimensional representation $\Theta(g)$ of $G$ with trivial factor system. Although the mathematical structure of the theory allows many nonequivalent factor groups for the representation $u(g)$, for physical systems only two factor groups are relevant: the trivial one, which applies to spinless particles, and the nontrivial factor system associated with the spinful particles. The representation $\Theta(g)$, which describes the difference between two representations with the same factor system, always has the trivial factor group.

The canonical form for the representation $u(g)$ is

$$
u(g)=\operatorname{diag}\left[r_{1}(g) \otimes \mathbb{1}_{N_{1}}, \ldots, r_{n}(g) \otimes \mathbb{1}_{N_{n}}\right],
$$

where the $r_{\alpha}(g)$ are irreducible representations ("irreps") of $G$ (with the appropriate factor system) and $\mathbb{1}_{N_{\alpha}}$ the $N_{\alpha} \times N_{\alpha}$ unit matrix. The dimension of $h(\mathbf{k})$, corresponding to the total number of orbitals in the unit cell, is $\sum_{\alpha} d_{\alpha} N_{\alpha}$, where $d_{\alpha}$ is the dimension of the irreducible presentation $\alpha$. For notational simplicity, we choose to set $N_{1}=N_{2}=\cdots=N_{n} \equiv N$.

The general symmetry constraint for the full BdG Hamiltonian $H(\mathbf{k})$ is obtained by allowing different realizations $u_{1,2}(g)$ of the transformation matrices for the particle and hole degrees of freedom. Such a symmetry constraint is compatible with the group operation of $G$ if $u_{1}(g)$ and $u_{2}(g)$ have the same factor system, i.e., if there exists a one-dimensional representation $\Theta(g)$ of the symmetry group $G$ with trivial factor system such that

$$
u_{1}(g) \equiv u(g)=u_{2}(g) \Theta(g) .
$$

For the BdG Hamiltonian $H(\mathbf{k})$ one then finds the general symmetry constraint

$$
H(\mathbf{k})=U(g) H(g \mathbf{k}) U(g)^{\dagger},
$$

with

$$
U(g)=\left(\begin{array}{cc}
u(g) & 0 \\
0 & u(g)^{*} \Theta(g)
\end{array}\right) .
$$

For the superconducting order parameter $\Delta(\mathbf{k})$, Eqs. (7) and (8) imply that

$$
\Delta(\mathbf{k})=u(g) \Delta(g \mathbf{k}) u(g)^{\mathrm{T}} \Theta(g)^{*},
$$

i.e., $\Delta(\mathbf{k})$ transforms under a one-dimensional representation of the group $G$ [69]. Whereas the projective representation $u(g)$ is a property of the normal phase, the additional phase factor $\Theta(g)$ exists by virtue of the superconducting order only. Together, the representation $u$ and the one-dimensional representation $\Theta$ fully determine the symmetry class of the Bogoliubov-de Gennes Hamiltonian (1).

Alternatively, Eqs. (7) and (8) may be recast in the form of an algebraic relation between the elements $g$ of the symmetry group $G$ and the particle-hole conjugation operation $\mathcal{P}$ :

$$
g \mathcal{P}=\Theta(g) \mathcal{P} g .
$$

This is the formulation used in Refs. [36,38,39,46,48,70], which considered the full classification of BdG Hamiltonians in the presence of a single order-two spatial symmetry $\mathcal{S}$ and discriminated between the cases in which $\mathcal{S}$ commutes or anticommutes with the Fermi constraint $\mathcal{P}$.

The presence of time-reversal symmetry and/or spinrotation symmetry does not change the above considerations, provided these commute with the crystalline symmetries. Time-reversal symmetry imposes the additional constraints [8]

$$
h(\mathbf{k})=\sigma_{2} h(-\mathbf{k})^{*} \sigma_{2}, \quad \Delta(\mathbf{k})=\sigma_{2} \Delta(-\mathbf{k})^{*} \sigma_{2},
$$

where $\sigma_{2}$ is a Pauli matrix that acts in spin space. With time-reversal symmetry, the one-dimensional representation $\Theta(g)$ of $G$ that characterizes the superconducting state must be real. In the presence of spin-rotation symmetry, one arrives at an effective description in terms of a BdG-type Hamiltonian of the form (1), but with a symmetric order parameter $\Delta(\mathbf{k})=\Delta(-\mathbf{k})^{\mathrm{T}}$, so that $H(\mathbf{k})$ is antisymmetric under the antiunitary operation $\tau_{2} K$, which plays the role of an effective particle-hole symmetry. If time-reversal symmetry is present 
TABLE I. Irreducible representations of the point group $C_{i}$. The second column lists the standard crystallographic notation for the representations, the first column gives the notation used in the text.

\begin{tabular}{cccc}
\hline \hline & $\Gamma$ & $\mathbf{1}$ & $\mathcal{I}_{\pi}$ \\
\hline$r_{+}$ & $A_{g}$ & 1 & 1 \\
$r_{-}$ & $A_{u}$ & 1 & -1 \\
\hline \hline
\end{tabular}

additionally, $H(\mathbf{k})$ is symmetric under complex conjugation $K$, which plays the role of an effective time-reversal operation. Note that while $\mathcal{P}$ and $\mathcal{T}$ square to 1 and -1 , respectively, in the spinful case, the effective particle-hole conjugation and time-reversal operations square to -1 and 1 , respectively, if spin-rotation symmetry is present. Similarly, in the presence of spin-rotation symmetry, the effective representation of crystalline symmetry operations is changed into the corresponding "spinless" type.

The general framework described here is best illustrated using examples. As a first example, we consider a system with inversion symmetry. In this case, the group $G=C_{i}=\{\mathbf{1}, \mathcal{I}\}$, where 1 is the identity and $\mathcal{I}$ is the inversion operation. Choosing a basis that has a well-defined parity under inversion, one finds that one may represent $G$ by

$$
u(g)=\operatorname{diag}\left[r_{+}(g), r_{-}(g)\right],
$$

where $r_{ \pm}(g)$ are the two irreducible representations of $G$ (see Table I), which are both one dimensional. Specifically, Eq. (12) reads as

$$
u(\mathbf{1})=\rho_{0}, \quad u(\mathcal{I})=\rho_{3},
$$

where $\rho_{3}$ is a Pauli matrix that acts in the space of even- and odd-parity states and $\rho_{0}$ is the $2 \times 2$ unit matrix. From the two one-dimensional representations of $G$ we also find two possibilities for the representation $U(g)$ for the BdG Hamiltonian $H(\mathbf{k})$. Choosing the trivial one-dimensional representation $\Theta(g)=r_{+}(g)$ in Eq. (7) (the $A_{g}$ representation, see Table I), we find that the BdG Hamiltonian $H(\mathbf{k})$ obeys the symmetry constraint

$$
H(\mathbf{k})=\left(\rho_{3} \tau_{0}\right) H(-\mathbf{k})\left(\rho_{3} \tau_{0}\right)
$$

where $\tau_{0}$ is the identity matrix in particle-hole space and $\rho_{3}$ is a Pauli matrix that acts in the space of even- and odd-parity states. For the blocks $h(\mathbf{k})$ and $\Delta(\mathbf{k})$ this implies

$$
h(\mathbf{k})=\rho_{3} h(-\mathbf{k}) \rho_{3}, \quad \Delta(\mathbf{k})=\rho_{3} \Delta(-\mathbf{k}) \rho_{3},
$$

i.e., the order parameter is even under inversion. Alternatively, choosing the nontrivial one-dimensional representation $\Theta(g)=u_{-}(g)$ in Eq. (7) (the $A_{u}$ representation), we find that the BdG Hamiltonian $H(\mathbf{k})$ satisfies

$$
H(\mathbf{k})=\left(\rho_{3} \tau_{3}\right) H(-\mathbf{k})\left(\rho_{3} \tau_{3}\right)
$$

In this case the blocks $h(\mathbf{k})$ and $\Delta(\mathbf{k})$ satisfy the constraint

$$
h(\mathbf{k})=\rho_{3} h(-\mathbf{k}) \rho_{3}, \quad \Delta(\mathbf{k})=-\rho_{3} \Delta(-\mathbf{k}) \rho_{3},
$$

so that the order parameter is odd under inversion. The two different transformation rules of the order parameter $\Delta(\mathbf{k})$ under inversion imply two different algebraic relations between the inversion operation $\mathcal{I}$ and the Fermi constraint $\mathcal{P}$ :
TABLE II. Spinless representations (top four rows) and onedimensional spinful representations (bottom four rows) of the point group $C_{4}$. The second column lists the standard crystallographic notation for the representations, the first column gives the notation used in the text.

\begin{tabular}{lcccrc}
\hline \hline & $\Gamma$ & $\mathbf{1}$ & $\mathcal{R}_{\pi / 2}$ & $\mathcal{R}_{\pi / 2}^{2}$ & $\mathcal{R}_{\pi / 2}^{3}$ \\
\hline$r_{0}$ & $A$ & 1 & 1 & 1 & 1 \\
$r_{\pi / 2}$ & ${ }^{2} E$ & 1 & $i$ & -1 & $-i$ \\
$r_{\pi}$ & $B$ & 1 & -1 & 1 & -1 \\
$r_{3 \pi / 2}$ & ${ }^{1} E$ & 1 & $-i$ & -1 & $i$ \\
\hline$r_{\pi / 4}$ & ${ }^{1} \bar{E}_{1}$ & 1 & $e^{i \pi / 4}$ & $i$ & $e^{3 \pi i / 4}$ \\
$r_{3 \pi / 4}$ & ${ }^{2} \bar{E}_{2}$ & 1 & $e^{3 \pi i / 4}$ & $-i$ & $e^{i \pi / 4}$ \\
$r_{5 \pi / 4}$ & ${ }^{1} \bar{E}_{2}$ & 1 & $e^{5 \pi i / 4}$ & $i$ & $e^{7 \pi i / 4}$ \\
$r_{7 \pi / 4}$ & ${ }^{2} \bar{E}_{1}$ & 1 & $e^{7 \pi i / 4}$ & $-i$ & $e^{5 \pi i / 4}$ \\
\hline \hline
\end{tabular}

If $\Delta(\mathbf{k})$ is even under $\mathcal{I}, \mathcal{I}$ and $\mathcal{P}$ commute, whereas $\mathcal{I}$ and $\mathcal{P}$ anticommute if $\Delta(\mathbf{k})$ is odd under inversion.

As a second example, we consider a fourfold rotation around a fixed axis for a system of spinful particles. We write $G=C_{4}=\left\{\mathbf{1}, \mathcal{R}_{\pi / 2}, \mathcal{R}_{\pi / 2}^{2}, \mathcal{R}_{\pi / 2}^{3}\right\}$, where the generator $\mathcal{R}_{\pi / 2} \equiv \mathcal{R}$ denotes a clockwise rotation by $\pi / 2$. In this case, one has $\mathcal{R}^{4}=\mathbf{- 1}$, which corresponds to the factor system

$$
z_{\mathcal{R}^{k}, \mathcal{R}^{l}}= \begin{cases}1 & \text { if } 0 \leqslant k+l<4 \\ -1 & \text { if } 4 \leqslant k+l<8\end{cases}
$$

Choosing basis states with well-defined angular momentum $j=\frac{1}{2}, \frac{3}{2}, \frac{5}{2}$, and $\frac{7}{2}$ (defined modulo 4 ), we find the spinful representation

$$
u(g)=\operatorname{diag}\left[r_{\pi / 4}(g), r_{3 \pi / 4}(g), r_{5 \pi / 4}(g), r_{7 \pi / 4}(g)\right]
$$

(see Table II).

The transformation rule for the BdG Hamiltonian $H(\mathbf{k})$ requires the choice of a a one-dimensional spinless representation $\Theta$ of $G$. There are four of those, and we denote these as $r_{\theta}$ with $\theta=0, \pi / 2, \pi$, and $3 \pi / 2$ (see Table II). Proceeding as before, we find four possible transformation rules for the BdG Hamiltonian $H(\mathbf{k})$ under a $\pi / 2$ rotation $\mathcal{R}$,

$$
H(\mathbf{k})=U_{\theta}(\mathcal{R}) H(\mathcal{R} \mathbf{k}) U_{\theta}(\mathcal{R})^{\dagger}
$$

with

$$
U_{\theta}(\mathcal{R})=\left(\begin{array}{cc}
u(\mathcal{R}) & 0 \\
0 & u(\mathcal{R})^{*} e^{i \theta}
\end{array}\right)
$$

Alternatively, the transformation rules for the blocks $h(\mathbf{k})$ and $\Delta(\mathbf{k})$ are

$$
\begin{aligned}
h(\mathbf{k}) & =u(\mathcal{R}) h(\mathcal{R} \mathbf{k}) u(\mathcal{R})^{\dagger}, \\
\Delta(\mathbf{k}) & =e^{i \theta} u(\mathcal{R}) \Delta(\mathcal{R} \mathbf{k}) u(\mathcal{R})^{\mathrm{T}},
\end{aligned}
$$

which corresponds to a superconducting order parameter with finite angular momentum.

The time-reversal symmetry operation is represented by $U(\mathcal{T}) K$, where the definition of $U(\mathcal{T})$ in the angular momen- 
TABLE III. Spinful representation (bottom row) and onedimensional spinless representations (top four rows) of the point group $C_{2 v}$. The representation $u$ is unique up to unitary transformations. The second column lists the standard crystallographic notation for the representations, the first column gives the notation used in the text.

\begin{tabular}{lrrrrr}
\hline \hline & $\Gamma$ & $\mathbf{1}$ & $\mathcal{R}_{\pi}$ & $\mathcal{M}_{x}$ & $\mathcal{M}_{y}$ \\
\hline$r_{++}$ & $A_{1}$ & 1 & 1 & 1 & 1 \\
$r_{--}$ & $A_{2}$ & 1 & 1 & -1 & -1 \\
$r_{-+}$ & $B_{1}$ & 1 & -1 & 1 & -1 \\
$r_{+-}$ & $B_{2}$ & 1 & -1 & -1 & 1 \\
\hline$u$ & $E$ & $\sigma_{0}$ & $i \sigma_{3}$ & $i \sigma_{1}$ & $i \sigma_{2}$ \\
\hline \hline
\end{tabular}

tum basis is

$$
U(\mathcal{T})=\left(\begin{array}{cccc}
0 & 0 & 0 & -i \\
0 & 0 & -i & 0 \\
0 & i & 0 & 0 \\
i & 0 & 0 & 0
\end{array}\right)
$$

Time-reversal commutes with $\mathcal{P}$ and satisfies the property $\mathcal{T}^{2}=-1$. Time-reversal symmetry imposes the additional conditions (11) on the blocks $h(\mathbf{k})$ and $\Delta(\mathbf{k})$. One easily verifies that these additional conditions allow for a nonzero superconducting order parameter $\Delta(\mathbf{k})$ for the real one-dimensional representations corresponding to $\theta=0$ or $\pi$ only.

As a third example, we consider spinful fermions in a system with two perpendicular mirror symmetries, $G=C_{2 v}$. We write the point-group elements as $G=\left\{\mathbf{1}, \mathcal{M}_{x}, \mathcal{M}_{y}, \mathcal{R}_{\pi}\right\}$, where $\mathcal{M}_{x}$ and $\mathcal{M}_{x}$ are mirror reflections in planes perpendicular to the $x$ and $y$ axis, respectively, and $\mathcal{R}_{\pi}=\mathcal{M}_{y} \mathcal{M}_{x}$ is a rotation around the $z$ axis by $\pi$. For spinful particles, the factor system is nontrivial with $\mathcal{M}_{x}^{2}=\mathcal{M}_{y}^{2}=\mathcal{R}_{\pi}^{2}=-1$. In that case, there is only one (projective) representation $u$ of $G$ (up to unitary transformations), which is two dimensional (see Table III). Superconducting phases are characterized by onedimensional representations of $G$ with a trivial factor system. There are four of these, denoted $r_{\sigma_{x}, \sigma_{y}}(g)$, with $\sigma_{x, y}= \pm 1$ (see Table III). Each of the one-dimensional representations of $G$ corresponds to a distinct symmetry of the superconducting order parameter $\Delta$. For example, for the one-dimensional representation $r_{--}$, the order parameter is odd under mirror reflections in the $y z$ and $x z$ planes and even under a twofold rotation around the $z$ axis.

\section{CLASSIFICATION OF ZERO-DIMENSIONAL HAMILTONIANS}

The cornerstone of the construction of symmetry-based indicators is the classification of zero-dimensional Hamiltonians with additional unitary symmetries. Concretely, the classification problem is that of a Hamiltonian $H$ with or without time-reversal symmetry, particle-hole antisymmetry, or chiral antisymmetry, and with additional unitary symmetries specified by the group $G$. The presence or absence of time-reversal symmetry, particle-hole antisymmetry, and chiral antisymmetry determines the tenfold-way (Altland-Zirnbauer) class and
TABLE IV. The tenfold-way symmetry classes are defined by the absence or presence of time-reversal symmetry, particle-hole antisymmetry, or chiral antisymmetry. The triple $\eta=\left(\eta_{\mathcal{T}}, \eta_{\mathcal{P}}, \eta_{\mathcal{C}}\right)$ (left column) indicates the presence or absence of these symmetries as well as the square of the symmetry operation if it is present. The second column gives the Cartan label for the symmetry class; the third column lists the corresponding classifying group $\mathfrak{K}_{\eta}[0]$ for zero-dimensional Hamiltonians. The rightmost column lists the topological invariant $v$, where $\mathfrak{N}$ is the number of eigenstates (Kramers pairs in class AII) with negative energy and $\mathfrak{p}$ is the Pfaffian invariant as defined in Eq. (40).

\begin{tabular}{lccc}
\hline \hline$\eta=\left(\eta_{\mathcal{T}}, \eta_{\mathcal{P}}, \eta_{\mathcal{C}}\right)$ & Cartan & $\mathfrak{K}[0]$ & $v$ \\
\hline$(0,0,0)$ & A & $\mathbb{Z}$ & $\mathfrak{N}$ \\
$(0,0,1)$ & AIII & 0 & \\
\hline$(1,0,0)$ & AI & $\mathbb{Z}$ & $\mathfrak{N}$ \\
$(1,1,1)$ & BDI & $\mathbb{Z}_{2}$ & $\mathfrak{p}$ \\
$(0,1,0)$ & D & $\mathbb{Z}_{2}$ & $\mathfrak{p}$ \\
$(-1,1,1)$ & DIII & 0 & - \\
$(-1,0,0)$ & AII & $\mathbb{Z}$ & $\mathfrak{N}$ \\
$(-1,-1,1)$ & CII & 0 & \\
$(0,-1,0)$ & C & 0 & \\
$(1,-1,1)$ & CI & 0 & \\
\hline \hline
\end{tabular}

is indicated by the triple [8]

$$
\eta=\left(\eta_{\mathcal{T}}, \eta_{\mathcal{P}}, \eta_{\mathcal{C}}\right)
$$

Here, $\eta_{\mathcal{T}}=\mathcal{T}^{2}= \pm 1$ if time-reversal symmetry is present and $\eta_{\mathcal{T}}=0$ if time-reversal symmetry is absent. Similarly, $\eta_{\mathcal{P}}=\mathcal{P}^{2}= \pm 1$ or 0 if particle-hole antisymmetry is present or absent and $\eta_{\mathcal{C}}=\mathcal{C}^{2}=1$ or 0 in the presence or absence of chiral antisymmetry, respectively. Table IV summarizes the tenfold-way classification and lists both the notation using the triple $\left(\eta_{\mathcal{T}}, \eta_{\mathcal{P}}, \eta_{\mathcal{C}}\right)$ and the Cartan labeling. If the context allows, we will use the Cartan label instead of the triple $\eta=\left(\eta_{\mathcal{T}}, \eta_{\mathcal{P}}, \eta_{\mathcal{C}}\right)$.

For physically relevant systems, the crystalline symmetry group $G$ commutes with the time-reversal operation $\mathcal{T}$, which is what we assume throughout. The algebraic relation between $G$ and the particle-hole conjugation operation $\mathcal{P}$ or the chiral operation $\mathcal{C}=\mathcal{P} T$ is characterized by a one-dimensional representation $\Theta(g)$ of $G$, as discussed in Sec. II.

With a suitable choice of basis, the (projective) representation $U(g)$ for a Bogoliubov-de Gennes Hamiltonian $H$ can be brought into a canonical form analogous to Eq. (5),

$$
U(g)=\operatorname{diag}\left[r_{1}(g) \otimes \mathbb{1}_{2 N_{1}}, \ldots, r_{n}(g) \otimes \mathbb{1}_{2 N_{n}}\right],
$$

where the $r_{\alpha}(g)$ are irreducible representations of $G$. To keep the notation simple, we make the choice $N_{1}=N_{2}=\cdots=$ $N_{n}=N$. The Bogoliubov-de Gennes Hamiltonian $H$ is then brought into a block-diagonal form

$$
H=\operatorname{diag}\left(\mathbb{1}_{d_{1}} \otimes H_{1}, \ldots, \mathbb{1}_{d_{n}} \otimes H_{n}\right),
$$

where $d_{\alpha}$ is the dimension of the irreducible representation $r_{\alpha}$. The diagonal blocks $H_{\alpha}$ have dimension $2 N$ and are no longer subject to additional unitary symmetries. However, because of the basis change involved in representing $U$ in the canonical form (25), the action of the fundamental symmetry operations $\mathcal{T}, \mathcal{P}$, and/or $\mathcal{C}$ on these blocks need not be the same as their 
action on the original Hamiltonian $H$ and different blocks may be related to each other by $\mathcal{T}, \mathcal{P}$, and/or $\mathcal{C}$. Hence, additional considerations are needed to determine which blocks are independent and to what tenfold-way class they belong, which we now discuss.

Time-reversal operation $\mathcal{T}$. If the irreducible representations $r_{\alpha}$ and $r_{\alpha}^{*}$ are the same (up to a unitary transformation), the time-reversal operation $\mathcal{T}$ acts within the diagonal block corresponding to $r_{\alpha}$ and takes the form of an effective timereversal operation for $H_{\alpha}$, but with a square $W_{\mathcal{T}}(\alpha)$ that may differ from $\eta_{\mathcal{T}}$. If the irreducible representations $r_{\alpha}$ and $r_{\alpha}^{*}$ are different, $\mathcal{T}$ interchanges the diagonal blocks corresponding to $r_{\alpha}$ and $r_{\alpha}^{*}$. In most examples, which of these cases applies can easily be found by inspection, although one may also obtain the answer from the "Wigner test" by calculating the quantity $[27,64,71]$

$$
W_{\mathcal{T}}(\alpha)=\eta_{\mathcal{T}}\left\langle z_{g, g} \chi_{\alpha}\left(g^{2}\right)\right\rangle_{G},
$$

where $\langle\ldots\rangle_{G}$ denotes an average over all $g \in G, z_{g, g}$ is an element of the factor system [see Eq. (4)], and $\chi_{\alpha}(g)=\operatorname{tr} r_{\alpha}(g)$ is the character of the irreducible representation. The quantity $W_{\mathcal{T}}(\alpha)$ can take the values $W_{\mathcal{T}}(\alpha)= \pm 1$ or 0 , corresponding to the two cases discussed above.

Particle-hole conjugation $\mathcal{P}$. Similarly, if the irreducible representations $r_{\alpha}$ and $\Theta r_{\alpha}^{*}$ are the same, $\mathcal{P}$ acts as an effective particle-hole conjugation operator for $H_{\alpha}$ with square $W_{\mathcal{P}}(\alpha)= \pm 1$, whereas if $r_{\alpha}$ and $\Theta r_{\alpha}^{*}$ are different irreducible representations of $G, \mathcal{P}$ interchanges the corresponding diagonal blocks. Again, one may find out which of the three cases applies by calculating

$$
W_{\mathcal{P}}(\alpha)=\eta_{\mathcal{P}}\left\langle\Theta(g)^{*} z_{g, g} \chi_{\alpha}\left(g^{2}\right)\right\rangle_{G},
$$

which takes the values \pm 1 or 0 , respectively, for the two cases described above.

Chiral operation $\mathcal{C}$. Finally, for the chiral operation $\mathcal{C}=$ $\mathcal{P} T$ one only needs to distinguish two cases: If $r_{\alpha}$ and $\Theta r_{\alpha}$ are the same irreducible representation of $G, \mathcal{C}$ acts as an effective antisymmetry of $H_{\alpha}$, whereas $\mathcal{C}$ interchanges the diagonal blocks corresponding to $r_{\alpha}$ and $\Theta r_{\alpha}$ if they are different. This defines the quantity $W_{\mathcal{C}}(\alpha)$, which takes the values 0 or 1 .

We denote the full classifying group for zero-dimensional Hamiltonians with the additional symmetry group $G$ as $\mathfrak{K}_{\eta}[G, \Theta]$, where the multi-index $\eta$ indicates the tenfoldway class (see Table IV), and $\Theta$ is the one-dimensional representation of $G$ that characterizes the superconducting order parameter. The classifying group also depends on the factor system of $G$, but we do not write this dependence explicitly. The argument $\Theta$ is omitted for nonsuperconducting phases. The above considerations then lead to the result

$$
\mathfrak{K}_{\eta}[G, \Theta]=\prod_{r_{\alpha} \text { irrep of } G} \mathfrak{K}_{W(\alpha)}[0],
$$

where the multi-index $W(\alpha)=\left(W_{\mathcal{T}}(\alpha), W_{\mathcal{P}}(\alpha), W_{\mathcal{C}}(\alpha)\right)$ and the product is over the irreducible representations $r_{\alpha}$ of $G$, where only one representative appears in the product if multiple irreps are linked by the fundamental symmetries $\mathcal{T}, \mathcal{P}$, or $\mathcal{C}$. The classifying groups $\mathfrak{K}_{\eta}[0]$, which apply to tenfold-way classes without additional crystalline symmetries [9,72], can be found in Table IV. We remark that the classification approach described here also works for $d$-dimensional Hamiltonians $H(\mathbf{k})$, as long as $G$ contains only onsite (i.e., nonspatial, $\mathbf{k}$-independent) crystalline symmetries.

Examples. We illustrate this general procedure using the three examples already discussed in the previous section. The classification results for these three examples as well as for other relevant point groups are summarized in Tables V-VIII.

We first consider a zero-dimensional superconductor with inversion symmetry. The symmetry group $G=C_{i}=\{\mathbf{1}, \mathcal{I}\}$ and the two one-dimensional representations $\Theta$ of $G$ that characterize the superconducting phase are the two irreducible representations $r_{ \pm}$of $G$ (see Table I). If the superconducting order is even under inversion, corresponding to $\Theta=r_{+}$(the " $A_{g}$ " representation), Eqs. (5) and (8) give

$$
U(\mathbf{1})=\rho_{0} \tau_{0}, \quad U(\mathcal{I})=\rho_{3} \tau_{0},
$$

where $\rho_{3}$ is the Pauli matrix in parity space. Correspondingly, the BdG Hamiltonian $H=\operatorname{diag}\left(H_{+}, H_{-}\right)$is the diagonal sum of blocks with even and odd parity under inversion. Since $U$ is real and does not involve the particle-hole degree of freedom, the particle-hole conjugation operation $\mathcal{P}$ commutes with $G$, so that it acts within each parity subblock. Its square is $W_{\mathcal{P}}=$ $\eta_{\mathcal{P}}$, i.e., the same as for the full Hamiltonian $H$. We conclude that

$$
\mathfrak{K}_{\eta}\left[C_{i}, A_{g}\right]=\mathfrak{K}_{\eta}[0]^{2}
$$

for all tenfold-way symmetry classes $\eta$. The groups $\mathfrak{K}_{\eta}[0]$ are listed in Table IV. This conclusion is consistent with the Wigner test, which gives $W_{\mathcal{P}}\left(r_{ \pm}\right)=1$. If, on the other hand, $\Delta$ is odd under inversion, which corresponds to the onedimensional representation $\Theta=r_{-}$for the superconducting phase [the " $A_{u}$ " representation (see Table I)], the representation of $G$ for the full BdG Hamiltonian is

$$
U(\mathbf{1})=\rho_{0} \tau_{0}, \quad U(\mathcal{I})=\rho_{3} \tau_{3}
$$

[see Eq. (14)]. The block-diagonal structure of $H$ now involves one block with even-parity particlelike states and oddparity holelike states and one block with odd-parity particlelike states and even-parity holelike states. The two blocks are interchanged by particle-hole conjugation. One arrives at the same conclusion by observing that $r_{+}$and $\Theta r_{+}=r_{-}$are different irreducible representations of $G$. For the classifying group $\mathfrak{K}_{\eta}\left[G, A_{u}\right]$ we thus find

$$
\mathfrak{K}_{\left(\eta_{\mathcal{T}}, \eta_{\mathcal{P}}, \eta_{\mathcal{C}}\right)}\left[C_{i}, A_{u}\right]=\mathfrak{K}_{\left(\eta_{\mathcal{T}}, 0,0\right)}[0] .
$$

Again, this conclusion is compatible with the Wigner test, which gives $W_{\mathcal{P}}\left(r_{ \pm}\right)=0$.

The second example deals with a spinful system with a fourfold rotation symmetry $G=C_{4}$. The one-dimensional representation $\Theta \equiv r_{\theta}$ of $G$ that characterizes the superconducting phase is labeled by an angle $\theta$ which can take the values $0, \pi / 2, \pi$, or $3 \pi / 2$ (see Table II). The representation (21) of the rotation generator $\mathcal{R}_{\pi / 2}$ implies that $H$ has a block-diagonal structure in which particlelike states with angular momentum $j=\frac{1}{2}, \frac{3}{2}, \frac{5}{2}$, or $\frac{7}{2}$ are combined with holelike states of angular momentum $-j+2 \theta / \pi \bmod 4$. In the following discussion, we use the angular momentum $j$ of the particlelike states to label the blocks. If $\theta=0$ (the " $A$ " 
TABLE V. Classification of zero-dimensional BdG Hamiltonians with point group $G$ and one-dimensional representation $\Theta$ describing the symmetry of the superconducting order parameter in the absence of time-reversal symmetry and spin-rotation symmetry, corresponding to tenfold-way class $\mathrm{D}, \eta_{\mathcal{P}}=1$. The second and third columns list the one-dimensional representation $\Theta$ using the standard crystallographic notation and the notation used in the examples in the main text, respectively. The fourth column lists the set $\left(r_{\alpha}\right)_{W_{\mathcal{P}}(\alpha)}$ of irreps $r_{\alpha}$ together with the result of the Wigner test $W_{\mathcal{P}}(\alpha)$. In case irreps $r_{\alpha}$ and $\Theta r_{\alpha}^{*}$ are paired by particle-hole antisymmetry $\left[W_{\mathcal{P}}(\alpha)=0\right]$, the paired representations appear in brackets. From this information one can read off the classification, invariants, generators, and representations using Table X. For convenience, we list the result for the classifying group $\mathfrak{K}_{\mathrm{D}}[G, \Theta]$ in the last column.

\begin{tabular}{|c|c|c|c|c|}
\hline$G$ & $\Theta$ & & $\left(r_{\alpha}\right)_{W_{\mathcal{P}}(\alpha)}$ & $\mathfrak{K}_{\mathrm{D}}[G, \Theta]$ \\
\hline$C_{1}$ & $A$ & 1 & $(A)_{\eta_{\mathcal{P}}}$ & $\mathbb{Z}_{2}$ \\
\hline$\overline{C_{i}}$ & $\begin{array}{l}A_{g} \\
A_{u}\end{array}$ & $\begin{array}{l}r_{+} \\
r_{-}\end{array}$ & $\begin{array}{c}\left(A_{g}\right)_{\eta_{\mathcal{P}}},\left(A_{u}\right)_{\eta_{\mathcal{P}}} \\
\left(A_{g}, A_{u}\right)_{0}\end{array}$ & $\begin{array}{l}\mathbb{Z}_{2}^{2} \\
\mathbb{Z}\end{array}$ \\
\hline$C_{s}$ & $\begin{array}{l}A^{\prime} \\
A^{\prime \prime}\end{array}$ & $\begin{array}{l}r_{+} \\
r_{-}\end{array}$ & $\begin{array}{c}\left({ }^{1} \bar{E},{ }^{2} \bar{E}\right)_{0} \\
\left({ }^{1} \bar{E}\right)_{\eta_{\mathcal{P}}},\left({ }^{2} \bar{E}\right)_{\eta_{\mathcal{P}}} \\
\end{array}$ & $\begin{array}{l}\mathbb{Z} \\
\mathbb{Z}_{2}^{2} \\
\end{array}$ \\
\hline$C_{2}$ & $\begin{array}{l}A \\
B \\
\end{array}$ & $\begin{array}{l}r_{+} \\
r_{-} \\
\end{array}$ & $\begin{array}{c}\left({ }^{1} \bar{E},{ }^{2} \bar{E}\right)_{0} \\
\left({ }^{1} \bar{E}\right)_{\eta_{\mathcal{P}}},\left({ }^{2} \bar{E}\right)_{\eta_{\mathcal{P}}}\end{array}$ & $\begin{array}{l}\mathbb{Z} \\
\mathbb{Z}_{2}^{2} \\
\end{array}$ \\
\hline$C_{3}$ & $\begin{array}{l}A_{1} \\
{ }^{2} E \\
{ }^{1} E\end{array}$ & $\begin{array}{c}r_{0} \\
r_{2 \pi / 3} \\
r_{4 \pi / 3} \\
\end{array}$ & $\begin{array}{l}\bar{E}_{\eta_{\mathcal{P}}},\left({ }^{1} \bar{E},{ }^{2} \bar{E}\right)_{0} \\
{ }^{2} \bar{E}_{\eta_{\mathcal{P}}},\left(\bar{E},{ }^{1} \bar{E}\right)_{0} \\
{ }^{1} \bar{E}_{\eta_{\mathcal{P}}},\left(\bar{E},{ }^{2} \bar{E}\right)_{0} \\
\end{array}$ & $\begin{array}{l}\mathbb{Z} \times \mathbb{Z}_{2} \\
\mathbb{Z} \times \mathbb{Z}_{2} \\
\mathbb{Z} \times \mathbb{Z}_{2}\end{array}$ \\
\hline$C_{4}$ & $\begin{array}{l}A \\
B \\
{ }^{2} E \\
{ }^{2} E\end{array}$ & $\begin{array}{c}r_{0} \\
r_{\pi} \\
r_{\pi / 2} \\
r_{3 \pi / 2}\end{array}$ & $\begin{array}{c}\left({ }^{1} \bar{E}_{1},{ }^{2} \bar{E}_{1}\right)_{0},\left({ }^{1} \bar{E}_{2},{ }^{2} \bar{E}_{2}\right)_{0} \\
\left({ }^{1} \bar{E}_{1},{ }^{2} \bar{E}_{2}\right)_{0},\left({ }^{1} \bar{E}_{2},{ }^{2} \bar{E}_{1}\right)_{0} \\
\left({ }^{1} \bar{E}_{1}\right)_{\eta_{\mathcal{P}}},\left({ }^{1} \bar{E}_{2}\right)_{\eta_{\mathcal{P}}},\left({ }^{2} \bar{E}_{1},{ }^{2} \bar{E}_{2}\right)_{0} \\
\left({ }^{2} \bar{E}_{1}\right)_{\eta_{\mathcal{P}}},\left({ }^{2} \bar{E}_{2}\right)_{\eta_{\mathcal{P}}},\left({ }^{1} \bar{E}_{1},{ }^{1} \bar{E}_{2}\right)_{0}\end{array}$ & $\begin{aligned} & \mathbb{Z}^{2} \\
& \mathbb{Z}^{2} \\
& \mathbb{Z} \times \mathbb{Z}_{2}^{2} \\
& \mathbb{Z} \times \mathbb{Z}_{2}^{2}\end{aligned}$ \\
\hline$C_{6}$ & $\begin{array}{c}A \\
B \\
{ }^{B} E_{1} \\
{ }^{2} E_{2} \\
{ }^{1} E_{1} \\
{ }^{1} E_{2}\end{array}$ & $\begin{array}{c}r_{0} \\
r_{\pi} \\
r_{4 \pi / 3} \\
r_{\pi / 3} \\
r_{2 \pi / 3} \\
r_{-\pi / 3}\end{array}$ & $\begin{array}{c}\left({ }^{1} \bar{E}_{1},{ }^{2} \bar{E}_{1}\right)_{0},\left({ }^{1} \bar{E}_{2},{ }^{2} \bar{E}_{2}\right)_{0},\left({ }^{1} \bar{E}_{3},{ }^{2} \bar{E}_{3}\right)_{0} \\
\left({ }^{1} \bar{E}_{1}\right)_{\eta_{\mathcal{P}}},\left({ }^{2} \bar{E}_{1}\right)_{\eta_{\mathcal{P}}},\left({ }^{1} \bar{E}_{3},{ }^{2} \bar{E}_{2}\right)_{0},\left({ }^{2} \bar{E}_{3},{ }^{1} \bar{E}_{2}\right)_{0} \\
\quad\left({ }^{1} \bar{E}_{1},{ }^{2} \bar{E}_{3}\right)_{0},\left({ }^{2} \bar{E}_{1},{ }^{1} \bar{E}_{2}\right)_{0},\left({ }^{2} \bar{E}_{2},{ }^{1} \bar{E}_{3}\right)_{0} \\
\left.\left({ }^{1} \bar{E}_{3}\right)_{\eta_{\mathcal{P}}},\left({ }^{2} \bar{E}_{2}\right)_{\eta_{\mathcal{P}},},{ }^{2} \bar{E}_{1},{ }^{2} \bar{E}_{3}\right)_{0},\left({ }^{1} \bar{E}_{1},{ }^{1} \bar{E}_{2}\right)_{0} \\
\left.\left({ }^{1} \bar{E}_{1},{ }^{2} \bar{E}_{2}\right)_{0},{ }^{2} \bar{E}_{1},{ }^{1} \bar{E}_{3}\right)_{0},\left({ }^{2} \bar{E}_{3},{ }^{1} \bar{E}_{2}\right)_{0} \\
\left({ }^{1} \bar{E}_{2}\right)_{\eta_{\mathcal{P}}},\left({ }^{2} \bar{E}_{3}\right)_{\eta_{\mathcal{P}}},\left({ }^{1} \bar{E}_{1},{ }^{1} \bar{E}_{3}\right)_{0},\left({ }^{2} \bar{E}_{1},{ }^{2} \bar{E}_{2}\right)_{0}\end{array}$ & $\begin{array}{l}\mathbb{Z}^{3} \\
\mathbb{Z}^{2} \times \mathbb{Z}_{2}^{2} \\
\mathbb{Z}^{3} \\
\mathbb{Z}^{2} \times \mathbb{Z}_{2}^{2} \\
\mathbb{Z}^{3} \\
\mathbb{Z}^{2} \times \mathbb{Z}_{2}^{2}\end{array}$ \\
\hline$\overline{C_{2 v}}$ & $\begin{array}{l}A_{1} \\
A_{2} \\
B_{1} \\
B_{2}\end{array}$ & $\begin{array}{l}r_{++} \\
r_{--} \\
r_{-+} \\
r_{+-}\end{array}$ & $\begin{array}{l}(\bar{E})_{-\eta_{\mathcal{P}}} \\
(\bar{E})_{\eta_{\mathcal{P}}} \\
(\bar{E})_{\eta_{\mathcal{P}}} \\
(\bar{E})_{\eta_{\mathcal{P}}}\end{array}$ & $\begin{array}{c}0 \\
\mathbb{Z}_{2} \\
\mathbb{Z}_{2} \\
\mathbb{Z}_{2}\end{array}$ \\
\hline$C_{3 v}$ & $\begin{array}{l}A_{1} \\
A_{2} \\
\end{array}$ & & $\begin{array}{c}\left({ }^{1} \bar{E},{ }^{2} \bar{E}\right)_{0},\left(\bar{E}_{1}\right)_{-\eta_{\mathcal{P}}} \\
\left({ }^{1} \bar{E}\right)_{\eta_{\mathcal{P}}},\left({ }^{2} \bar{E}\right)_{\eta_{\mathcal{P}}},\left(\bar{E}_{1}\right)_{\eta_{\mathcal{P}}}\end{array}$ & $\begin{array}{l}\mathbb{Z} \\
\mathbb{Z}_{2}^{3} \\
\end{array}$ \\
\hline$C_{4 v}$ & $\begin{array}{l}A_{1} \\
A_{2} \\
B_{1} \\
B_{2}\end{array}$ & & $\begin{array}{c}\left(\bar{E}_{1}\right)_{-\eta_{\mathcal{P}}},\left(\bar{E}_{2}\right)_{-\eta_{\mathcal{P}}} \\
\left(\bar{E}_{1}\right)_{\eta_{\mathcal{P}}},\left(\bar{E}_{2}\right)_{\eta_{\mathcal{P}}} \\
\left(\bar{E}_{1}, \bar{E}_{2}\right)_{0} \\
\left(\bar{E}_{1}, \bar{E}_{2}\right)_{0}\end{array}$ & $\begin{array}{l}0 \\
\mathbb{Z}_{2}^{2} \\
\mathbb{Z} \\
\mathbb{Z}\end{array}$ \\
\hline$\overline{C_{6 v}}$ & $\begin{array}{l}A_{1} \\
A_{2} \\
B_{1} \\
B_{2}\end{array}$ & & $\begin{array}{c}\left(\bar{E}_{1}\right)_{-\eta_{\mathcal{P}}},\left(\bar{E}_{2}\right)_{-\eta_{\mathcal{P}}},\left(\bar{E}_{3}\right)_{-\eta_{\mathcal{P}}} \\
\left(\bar{E}_{1}\right)_{\eta_{\mathcal{P}}},\left(\bar{E}_{2}\right)_{\eta_{\mathcal{P}}},\left(\bar{E}_{3}\right)_{\eta_{\mathcal{P}}} \\
\left(\bar{E}_{3}\right)_{1},\left(\bar{E}_{1}, \bar{E}_{2}\right)_{0} \\
\left(\bar{E}_{3}\right)_{1},\left(\bar{E}_{1}, \bar{E}_{2}\right)_{0}\end{array}$ & $\begin{array}{c}0 \\
\mathbb{Z}_{2}^{3} \\
\mathbb{Z} \times \mathbb{Z}_{2} \\
\mathbb{Z} \times \mathbb{Z}_{2}\end{array}$ \\
\hline
\end{tabular}

representation), particle-hole conjugation $\mathcal{P}$ interchanges the blocks with $j=\frac{1}{2}$ and $\frac{7}{2}$, as well as the blocks with $j=\frac{3}{2}$ and $\frac{5}{2}$. If $\theta=\pi / 2$ (the " $2 E$ " representation), $\mathcal{P}$ interchanges the blocks with $j=\frac{3}{2}$ and $\frac{7}{2}$, but acts within the blocks with $j=\frac{1}{2}$ or $\frac{5}{2}$, squaring to one. If $\theta=\pi$ (the " $A$ " representation), $\mathcal{P}$ interchanges the blocks with $j=\frac{1}{2}$ and $\frac{3}{2}$, as well as the blocks with $j=\frac{5}{2}$ and $\frac{7}{2}$. Finally, if $\theta=3 \pi / 2$ (the " $E$ " representation), $\mathcal{P}$ interchanges the blocks with $j=\frac{1}{2}$ and $\frac{5}{2}$, but acts within the blocks with $j=\frac{3}{2}$ and $\frac{7}{2}$, again squaring to one. We conclude that

$$
\begin{aligned}
\mathfrak{K}_{\mathrm{D}}\left[C_{4}, A\right] & =\mathfrak{K}_{\mathrm{D}}\left[C_{4}, B\right] \\
& =\mathfrak{K}_{\mathrm{A}}[0]^{2} \\
& =\mathbb{Z}^{2}
\end{aligned}
$$


TABLE VI. Same as Table V, but in the presence of spin-rotation symmetry, i.e., for spinless representations of the crystalline symmetry group. BdG Hamiltonians with spin-rotation symmetry and without time-reversal symmetry correspond to tenfold-way class $\mathrm{C}, \eta_{\mathcal{P}}=-1$.

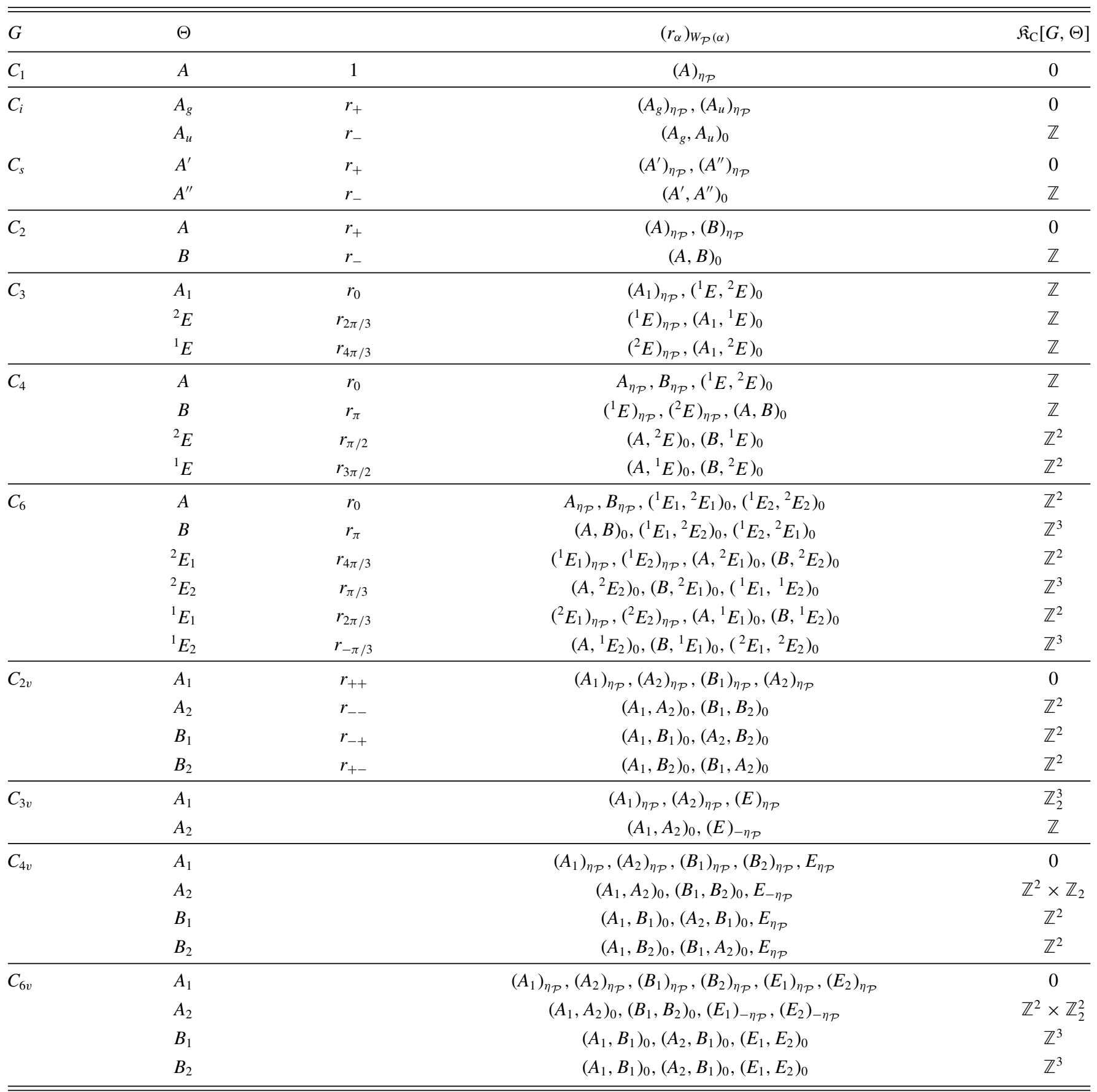

and

$$
\begin{aligned}
\mathfrak{K}_{\mathrm{D}}\left[C_{4},{ }^{1,2} E\right] & =\mathfrak{K}_{\mathrm{A}}[0] \times \mathfrak{K}_{\mathrm{D}}[0]^{2} \\
& =\mathbb{Z} \times \mathbb{Z}_{2}^{2} .
\end{aligned}
$$

The same conclusions can be obtained by performing the Wigner test.

In the presence of time-reversal symmetry only the real one-dimensional representations $A$ and $B$ are relevant (corresponding to $\theta=0, \pi$, respectively). In both cases, the timereversal operation $\mathcal{T}$ interchanges the blocks with angular momentum $j$ and angular momentum $-j \bmod 4$. For the $A$ representation, $\mathcal{P}$ and $\mathcal{T}$ give the same pairing of diagonal blocks of $H$ and the combined operation $\mathcal{C}=\mathcal{P} T$ leaves the diagonal blocks invariant. It follows that

$$
\begin{aligned}
\mathfrak{K}_{\text {DIII }}\left[C_{4}, A\right] & =\mathfrak{K}_{\mathrm{A}}[0]^{2} \\
& =0 .
\end{aligned}
$$

On the other hand, for the $B$ representation, $\mathcal{P}, \mathcal{T}$, and their product $\mathcal{C}=\mathcal{P} T$ map all four diagonal blocks of $H$ to each other and one has

$$
\begin{aligned}
\mathfrak{K}_{\text {DIII }}\left[C_{4}, B\right] & =\mathfrak{K}_{\mathrm{A}}[0] \\
& =\mathbb{Z} .
\end{aligned}
$$


TABLE VII. Classification of zero-dimensional BdG Hamiltonians with point group $G$ and one-dimensional representation $\Theta$ describing the symmetry of the superconducting order parameter with time-reversal symmetry without spin-rotation symmetry, corresponding to tenfoldway classes DIII, $\eta=(-1,1,1)$. We also include systems with emergent time-reversal symmetry, corresponding to class BDI, $\eta=(1,1,1)$. The second and third columns list the one-dimensional representation using the standard crystallographic notation and in the notation used in the examples in the main text, respectively. The fourth column lists the set $\left(r_{\alpha}\right)_{W_{\mathcal{P}}(\alpha)}$ of irreps $r_{\alpha}$, together with the result of the Wigner tests $W(\alpha)$. Irreps that are connected by application of the fundamental symmetry operations $\mathcal{T}, \mathcal{P}$, or $\mathcal{C}$ are shown together, using brackets. If two of the three Wigner tests $W_{\mathcal{T}}(\alpha), W_{\mathcal{C}}(\alpha)$, and $W_{\mathcal{C}}(\alpha)$ are zero, the irreps form a pair and the single symmetry operation that leaves the irrep invariant is the one with the nonzero Wigner label $W(\alpha)$. If all three Wigner tests are zero, $W_{\mathcal{T}}(\alpha)=W_{\mathcal{P}}(\alpha)=W_{\mathcal{C}}(\alpha)=0$, the irreps form a quartet. In that case, the order of the four irreps between brackets is such that the first two and last two irreps are interchanged by $\mathcal{T}$ and the first and third, and second and fourth irreps are interchanged by $\mathcal{P}$. From this information one can read off the classification, invariants, generators, and representations using Table IX. For convenience we list the result for the classifying group $\mathfrak{K}_{\eta}[G, \Theta]$ in the last two columns.

\begin{tabular}{|c|c|c|c|c|c|}
\hline$G$ & $\Theta$ & & $\left(r_{\alpha}\right)_{W_{\mathcal{P}}(\alpha)}$ & $\mathfrak{K}_{\mathrm{DIII}}[G, \Theta]$ & $\mathfrak{K}_{\mathrm{BDI}}[G, \Theta]$ \\
\hline$C_{1}$ & $A$ & 1 & $(A)_{\left(\eta_{\mathcal{T}}, \eta_{\mathcal{P}}, 1\right)}$ & 0 & $\mathbb{Z}_{2}$ \\
\hline \multirow[t]{2}{*}{$\overline{C_{i}}$} & $A_{g}$ & $r_{+}$ & $\left(A_{g}\right)_{\left(\eta_{\mathcal{T}}, \eta_{\mathcal{P}}, 1\right)},\left(A_{u}\right)_{\left(\eta_{\mathcal{T}}, \eta_{\mathcal{P}}, 1\right)}$ & 0 & $\mathbb{Z}_{2}^{2}$ \\
\hline & $A_{u}$ & $r_{-}$ & $\left(A_{g}, A_{u}\right)_{\left(\eta_{\mathcal{T}}, 0,0\right)}$ & $\mathbb{Z}$ & $\mathbb{Z}$ \\
\hline \multirow[t]{2}{*}{$C_{s}$} & $A^{\prime}$ & $r_{+}$ & $\left({ }^{1} \bar{E},{ }^{2} \bar{E}\right)_{(0,0,1)}$ & 0 & 0 \\
\hline & $A^{\prime \prime}$ & $r_{-}$ & $\left({ }^{1} \bar{E},{ }^{2} \bar{E}\right)_{\left(0, \eta_{\mathcal{P}}, 0\right)}$ & $\mathbb{Z}_{2}$ & $\mathbb{Z}_{2}$ \\
\hline \multirow[t]{2}{*}{$C_{2}$} & $A$ & $r_{+}$ & $\left({ }^{1} \bar{E},{ }^{2} \bar{E}\right)_{(0,0,1)}$ & 0 & 0 \\
\hline & $B$ & $r_{-}$ & $\left({ }^{1} \bar{E},{ }^{2} \bar{E}\right)_{\left(0, \eta_{\mathcal{P}}, 0\right)}$ & $\mathbb{Z}_{2}$ & $\mathbb{Z}_{2}$ \\
\hline$C_{3}$ & $A_{1}$ & $r_{0}$ & $\bar{E}_{\left(\eta_{\mathcal{T}}, \eta_{\mathcal{P}}, 1\right)},\left({ }^{1} \bar{E},{ }^{2} \bar{E}\right)_{(0,0,1)}$ & 0 & $\mathbb{Z}_{2}$ \\
\hline \multirow[t]{2}{*}{$C_{4}$} & $A$ & $r_{0}$ & $\left({ }^{1} \bar{E}_{1},{ }^{2} \bar{E}_{1}\right)_{(0,0,1)},\left({ }^{1} \bar{E}_{2},{ }^{2} \bar{E}_{2}\right)_{(0,0,1)}$ & 0 & 0 \\
\hline & $B$ & $r_{\pi}$ & $\left({ }^{1} \bar{E}_{1},{ }^{2} \bar{E}_{1},{ }^{2} \bar{E}_{2},{ }^{1} \bar{E}_{2}\right)_{(0,0,0)}$ & $\mathbb{Z}$ & $\mathbb{Z}$ \\
\hline \multirow[t]{2}{*}{$C_{6}$} & $A$ & $r_{0}$ & $\left({ }^{(1} \bar{E}_{1},{ }^{2} \bar{E}_{1}\right)_{(0,0,1)},\left({ }^{1} \bar{E}_{2},{ }^{2} \bar{E}_{2}\right)_{(0,0,1)},\left({ }^{1} \bar{E}_{3},{ }^{2} \bar{E}_{3}\right)_{(0,0,1)}$ & 0 & 0 \\
\hline & $B$ & $r_{\pi}$ & $\left({ }^{1} \bar{E}_{1},{ }^{2} \bar{E}_{1}\right)_{\left(0, \eta_{\mathcal{P}}, 0\right)},\left({ }^{1} \bar{E}_{2},{ }^{2} \bar{E}_{2},{ }^{2} \bar{E}_{3},{ }^{1} \bar{E}_{3}\right)_{(0,0,0)}$ & $\mathbb{Z} \times \mathbb{Z}_{2}$ & $\mathbb{Z} \times \mathbb{Z}_{2}$ \\
\hline \multirow[t]{4}{*}{$C_{2 v}$} & $A_{1}$ & $r_{++}$ & $(\bar{E})_{\left(-\eta_{\mathcal{T}},-\eta_{\mathcal{P}}, 1\right)}$ & 0 & 0 \\
\hline & $A_{2}$ & $r_{--}$ & $(\bar{E})_{\left(-\eta_{\mathcal{T}}, \eta_{\mathcal{P}}, 1\right)}$ & $\mathbb{Z}_{2}$ & 0 \\
\hline & $B_{1}$ & $r_{-+}$ & $(\bar{E})_{\left(-\eta_{\mathcal{T}}, \eta_{\mathcal{P}}, 1\right)}$ & $\mathbb{Z}_{2}$ & 0 \\
\hline & $B_{2}$ & $r_{+-}$ & $(\bar{E})_{\left(-\eta_{\mathcal{T}}, \eta_{\mathcal{P}}, 1\right)}$ & $\mathbb{Z}_{2}$ & 0 \\
\hline \multirow[t]{2}{*}{$C_{3 v}$} & $A_{1}$ & & $\left({ }^{1} \bar{E},{ }^{2} \bar{E}\right)_{(0,0,1)},\left(\bar{E}_{1}\right)_{\left(-\eta_{\mathcal{T}},-\eta_{\mathcal{P}}, 1\right)}$ & 0 & 0 \\
\hline & $A_{2}$ & & $\left({ }^{1} \bar{E},{ }^{2} \bar{E}\right)_{\left(0, \eta_{\mathcal{P}}, 0\right)},\left(\bar{E}_{1}\right)_{\left(-\eta_{\mathcal{T},}, \eta_{\mathcal{P}}, 1\right)}$ & $\mathbb{Z}_{2}^{2}$ & $\mathbb{Z}_{2}$ \\
\hline \multirow[t]{4}{*}{$C_{4 v}$} & $A_{1}$ & & $\left(\bar{E}_{1}\right)_{\left(-\eta_{\mathcal{T}},-\eta_{\mathcal{P}}, 1\right)},\left(\bar{E}_{2}\right)_{\left(-\eta_{\mathcal{T}},-\eta_{\mathcal{P}}, 1\right)}$ & 0 & 0 \\
\hline & $A_{2}$ & & $\left(\bar{E}_{1}\right)_{\left(-\eta_{\mathcal{T}}, \eta_{\mathcal{P}}, 1\right)},\left(\bar{E}_{2}\right)_{\left(-\eta_{\mathcal{T}}, \eta_{\mathcal{P}}, 1\right)}$ & $\mathbb{Z}_{2}^{2}$ & 0 \\
\hline & $B_{1}$ & & $\left(\bar{E}_{1}, \bar{E}_{2}\right)_{\left(-\eta_{\mathcal{T}}, 0,0\right)}$ & $\mathbb{Z}$ & $\mathbb{Z}$ \\
\hline & $B_{2}$ & & $\left(\bar{E}_{1}, \bar{E}_{2}\right)_{\left(-\eta \eta_{\mathcal{T}}, 0,0\right)}$ & $\mathbb{Z}$ & $\mathbb{Z}$ \\
\hline \multirow[t]{4}{*}{$C_{6 v}$} & $A_{1}$ & & $\left(\bar{E}_{1}\right)_{\left(-\eta_{\mathcal{T}},-\eta_{\mathcal{P}}, 1\right)},\left(\bar{E}_{2}\right)_{\left(-\eta_{\mathcal{T}},-\eta_{\mathcal{P}}, 1\right)},\left(\bar{E}_{3}\right)_{\left(-\eta_{\mathcal{T}},-\eta_{\mathcal{P}}, 1\right)}$ & 0 & 0 \\
\hline & $A_{2}$ & & $\left(\bar{E}_{1}\right)_{\left(-\eta_{\mathcal{T}}, \eta_{\mathcal{P}}, 1\right)},\left(\bar{E}_{2}\right)_{\left(-\eta_{\mathcal{T}}, \eta_{\mathcal{P}}, 1\right)},\left(\bar{E}_{3}\right)_{\left(-\eta_{\mathcal{T}}, \eta_{\mathcal{P}}, 1\right)}$ & $\mathbb{Z}_{2}^{3}$ & 0 \\
\hline & $B_{1}$ & & $\left(\bar{E}_{3}\right)_{\left(-\eta_{\mathcal{T}}, \eta_{\mathcal{P}}, 1\right)},\left(\bar{E}_{1}, \bar{E}_{2}\right)_{\left(-\eta_{\mathcal{T}}, 0,0\right)}$ & $\mathbb{Z} \times \mathbb{Z}_{2}$ & $\mathbb{Z}$ \\
\hline & $B_{2}$ & & $\left(\bar{E}_{3}\right)_{\left(-\eta_{\mathcal{T}}, \eta_{\mathcal{P}}, 1\right)},\left(\bar{E}_{1}, \bar{E}_{2}\right)_{\left(-\eta_{\mathcal{T}}, 0,0\right)}$ & $\mathbb{Z} \times \mathbb{Z}_{2}$ & $\mathbb{Z}$ \\
\hline
\end{tabular}

The third example is that of spinful particles with symmetry group $G=C_{2 v}$. We refer to Table III for the irreducible representations with nontrivial and trivial factor system. Since there is only one (two-dimensional) irreducible representation $u$ [the " $E$ " representation (see Table III)], it follows automatically that $u^{*}$ and $r_{\sigma_{x} \sigma_{y}} u^{*}$ are the same representations of $G$. Indeed, one easily verifies that $u^{*}$ and $r_{\sigma_{x}, \sigma_{y}} u^{*}$ are equal to $u$ up to a unitary transformation. To find the square of the effective time-reversal and particle-hole conjugation operations, we consider the case of the one-dimensional irreducible representation $\Theta=r_{++}$(the $A_{1}$ representation) in detail and summarize results for the remaining three one-dimensional representations $r_{+-}, r_{-+}$, and $r_{--}$(the $B_{2}, B_{1}$, and $A_{2}$ representations, respectively).
For $\Theta=r_{++}\left[“ A_{1}\right.$ ” representation (see Table III)], and choosing the representation of Table III for the representation of the generators $\mathcal{M}_{x}$ and $\mathcal{M}_{y}$ for the normal-state block $h$, we find that their representation for the full BdG Hamiltonian $H$ are $U\left(\mathcal{M}_{x}\right)=i \sigma_{1} \tau_{3}$ and $U\left(\mathcal{M}_{y}\right)=i \sigma_{2} \tau_{0}$. To bring the representation matrices to the canonical form (25) we have to perform the basis transformation

$$
H \rightarrow\left(\begin{array}{cc}
1 & 0 \\
0 & i \sigma_{2}
\end{array}\right) H\left(\begin{array}{cc}
1 & 0 \\
0 & -i \sigma_{2}
\end{array}\right)
$$

After this basis transformation, $\mathcal{M}_{x}$ and $\mathcal{M}_{y}$ are represented as $i \sigma_{1} \tau_{0}$ and $i \sigma_{2} \tau_{0}$, respectively, consistent with Eq. (25). The particle-hole conjugation operation $\mathcal{P}$, which was represented 
TABLE VIII. Same as Table VII, but in the presence of spin-rotation symmetry, i.e., for spinless representations of the crystalline symmetry group. Time-reversal symmetric BdG Hamiltonians with spin-rotation symmetry correspond to tenfold-way class CI, $\eta=(1,-1,1)$. We also include systems with emergent time-reversal symmetry, corresponding to class CII, $\eta=(-1,-1,1)$.

\begin{tabular}{|c|c|c|c|c|c|}
\hline$G$ & $\Theta$ & & $\left(r_{\alpha}\right)_{W_{\mathcal{P}}(\alpha)}$ & $\mathfrak{K}_{\mathrm{CI}}[G, \Theta]$ & $\mathfrak{K}_{\mathrm{CII}}[G, \Theta]$ \\
\hline$C_{1}$ & $A$ & 1 & $(A)_{\left(\eta_{\mathcal{T}}, \eta_{\mathcal{P}}, 1\right)}$ & 0 & 0 \\
\hline \multirow[t]{2}{*}{$C_{i}$} & $A_{g}$ & $r_{+}$ & $\left(A_{g}\right)_{\left(\eta_{\mathcal{T}}, \eta_{\mathcal{P}}, 1\right)},\left(A_{u}\right)_{\left(\eta_{\mathcal{T}}, \eta_{\mathcal{P}}, 1\right)}$ & 0 & 0 \\
\hline & $A_{u}$ & $r_{-}$ & $\left(A_{g}, A_{u}\right)_{\left(\eta_{\mathcal{T}}, 0,0\right)}$ & $\mathbb{Z}$ & $\mathbb{Z}$ \\
\hline \multirow[t]{2}{*}{$C_{s}$} & $A^{\prime}$ & $r_{+}$ & $\left(A^{\prime}\right)_{\left(\eta_{\mathcal{T}}, \eta_{\mathcal{P}}, 1\right)},\left(A^{\prime \prime}\right)_{\left(\eta_{\mathcal{T},}, \eta_{\mathcal{P}}, 1\right)}$ & 0 & 0 \\
\hline & $A^{\prime \prime}$ & $r_{-}$ & $\left(A^{\prime}, A^{\prime \prime}\right)_{\left(\eta_{\mathcal{T}}, 0,0\right)}$ & $\mathbb{Z}$ & $\mathbb{Z}$ \\
\hline \multirow[t]{2}{*}{$C_{2}$} & $A$ & $r_{+}$ & $(A)_{\left(\eta_{\mathcal{T}}, \eta_{\mathcal{P}}, 1\right)},(B)_{\left(\eta_{\mathcal{T}}, \eta_{\mathcal{P}}, 1\right)}$ & 0 & 0 \\
\hline & $B$ & $r_{-}$ & $(A, B)_{\left(\eta_{\mathcal{T}}, 0,0\right)}$ & $\mathbb{Z}$ & $\mathbb{Z}$ \\
\hline$C_{3}$ & $A_{1}$ & $r_{0}$ & $\left(A_{1}\right)_{\left(\eta_{\mathcal{T}}, \eta_{\mathcal{P}}, 1\right)},\left({ }^{1} E,{ }^{2} E\right)_{(0,0,1)}$ & 0 & 0 \\
\hline \multirow[t]{2}{*}{$\overline{C_{4}}$} & $A$ & $r_{0}$ & $A_{\left(\eta_{\mathcal{T}}, \eta_{\mathcal{P}}, 1\right)}, B_{\left(\eta_{\mathcal{T}}, \eta_{\mathcal{P}}, 1\right)},\left({ }^{1} E,{ }^{2} E\right)_{(0,0,1)}$ & 0 & 0 \\
\hline & $B$ & $r_{\pi}$ & $\left({ }^{1} E,{ }^{2} E\right)_{\left(0, \eta_{\mathcal{P}}, 0\right)},(A, B)_{\left(\eta_{\mathcal{T}}, 0,0\right)}$ & $\mathbb{Z}$ & $\mathbb{Z}$ \\
\hline \multirow[t]{2}{*}{$C_{6}$} & $A$ & $r_{0}$ & $A_{\left(\eta_{\mathcal{T}}, \eta_{\mathcal{P}}, 1\right)}, B_{\left(\eta_{\mathcal{T}}, \eta_{\mathcal{P}}, 1\right)},\left({ }^{1} E_{1},{ }^{2} E_{1}\right)_{(0,0,1)},\left({ }^{1} E_{2},{ }^{2} E_{2}\right)_{(0,0,1)}$ & 0 & 0 \\
\hline & $B$ & $r_{\pi}$ & $(A, B)_{\left(\eta_{\mathcal{T}}, 0,0\right)},\left({ }^{1} E_{1},{ }^{2} E_{1},{ }^{2} E_{2},{ }^{1} E_{2}\right)_{(0,0,0)}$ & $\mathbb{Z}^{2}$ & $\mathbb{Z}^{2}$ \\
\hline \multirow[t]{4}{*}{$\overline{C_{2 v}}$} & $A_{1}$ & $r_{++}$ & $\left(A_{1}\right)_{\left(\eta_{\mathcal{T}}, \eta_{\mathcal{P}}, 1\right)},\left(A_{2}\right)_{\left(\eta_{\mathcal{T}}, \eta_{\mathcal{P}}, 1\right)},\left(B_{1}\right)_{\left(\eta_{\mathcal{T}}, \eta_{\mathcal{P}}, 1\right)},\left(A_{2}\right)_{\left(\eta_{\mathcal{T}}, \eta_{\mathcal{P}}, 1\right)}$ & 0 & 0 \\
\hline & $A_{2}$ & $r_{--}$ & $\left(A_{1}, A_{2}\right)_{\left(\eta_{\mathcal{T}}, 0,0\right)},\left(B_{1}, B_{2}\right)_{\left(\eta_{\mathcal{T}}, 0,0\right)}$ & $\mathbb{Z}^{2}$ & $\mathbb{Z}^{2}$ \\
\hline & $B_{1}$ & $r_{-+}$ & $\left(A_{1}, B_{1}\right)_{\left(\eta_{\mathcal{T}}, 0,0\right)},\left(A_{2}, B_{2}\right)_{\left(\eta_{\mathcal{T}}, 0,0\right)}$ & $\mathbb{Z}^{2}$ & $\mathbb{Z}^{2}$ \\
\hline & $B_{2}$ & $r_{+-}$ & $\left(A_{1}, B_{2}\right)_{\left(\eta_{\mathcal{T}}, 0,0\right)},\left(B_{1}, A_{2}\right)_{\left(\eta_{\mathcal{T}}, 0,0\right)}$ & $\mathbb{Z}^{2}$ & $\mathbb{Z}^{2}$ \\
\hline \multirow[t]{2}{*}{$C_{3 v}$} & $A_{1}$ & & $\left(A_{1}\right)_{\left(\eta_{\mathcal{T}}, \eta_{\mathcal{P}}, 1\right)},\left(A_{2}\right)_{\left(\eta_{\mathcal{T}}, \eta_{\mathcal{P}}, 1\right)},(E)_{\left(\eta_{\mathcal{T}}, \eta_{\mathcal{P}}, 1\right)}$ & 0 & 0 \\
\hline & $A_{2}$ & & $\left(A_{1}, A_{2}\right)_{\left(\eta_{\mathcal{T}}, 0,0\right)},(E)_{\left(\eta_{\mathcal{T}},-\eta_{\mathcal{P}}, 1\right)}$ & $\mathbb{Z} \times \mathbb{Z}_{2}$ & $\mathbb{Z}$ \\
\hline \multirow[t]{4}{*}{$\overline{C_{4 v}}$} & $A_{1}$ & & $\left(A_{1}\right)_{\left(\eta_{\mathcal{T}}, \eta_{\mathcal{P}}, 1\right)},\left(A_{2}\right)_{\left(\eta_{\mathcal{T}}, \eta_{\mathcal{P}}, 1\right)},\left(B_{1}\right)_{\left(\eta_{\mathcal{T}}, \eta_{\mathcal{P}}, 1\right)},\left(A_{2}\right)_{\left(\eta_{\mathcal{T}}, \eta_{\mathcal{P}}, 1\right)}, E_{\left(\eta_{\mathcal{T}}, \eta_{\mathcal{P}}, 1\right)}$ & 0 & 0 \\
\hline & $A_{2}$ & & $\left(A_{1}, A_{2}\right)_{\left(\eta_{\mathcal{T}}, 0,0\right)},\left(B_{1}, B_{2}\right)_{\left(\eta_{\mathcal{T}}, 0,0\right)}, E_{\left(\eta_{\mathcal{T}},-\eta_{\mathcal{P}}, 1\right)}$ & $\mathbb{Z}^{2} \times \mathbb{Z}_{2}$ & $\mathbb{Z}^{2}$ \\
\hline & $B_{1}$ & & $\left(A_{1}, B_{1}\right)_{\left(\eta_{\mathcal{T}}, 0,0\right)},\left(A_{2}, B_{1}\right)_{\left(\eta_{\mathcal{T}}, 0,0\right)}, E_{\left(\eta_{\mathcal{T}}, \eta_{\mathcal{P}}, 1\right)}$ & $\mathbb{Z}^{2}$ & $\mathbb{Z}^{2}$ \\
\hline & $B_{2}$ & & $\left(A_{1}, B_{2}\right)_{\left(\eta_{\mathcal{T}}, 0,0\right)},\left(B_{1}, A_{2}\right)_{\left(\eta_{\mathcal{T}}, 0,0\right)}, E_{\left(\eta_{\mathcal{T}}, \eta_{\mathcal{P}}, 1\right)}$ & $\mathbb{Z}^{2}$ & $\mathbb{Z}^{2}$ \\
\hline \multirow[t]{4}{*}{$\overline{C_{6 v}}$} & $A_{1}$ & & $\left(A_{1}\right)_{\left(\eta_{\mathcal{T}}, \eta_{\mathcal{P}}, 1\right)},\left(A_{2}\right)_{\left(\eta_{\mathcal{T}}, \eta_{\mathcal{P}}, 1\right)},\left(B_{1}\right)_{\left(\eta_{\mathcal{T}}, \eta_{\mathcal{P}}, 1\right)},\left(A_{2}\right)_{\left(\eta_{\mathcal{T}}, \eta_{\mathcal{P}}, 1\right)},\left(E_{1}\right)_{\left(\eta_{\mathcal{T}}, \eta_{\mathcal{P}}, 1\right)},\left(E_{2}\right)_{\left(\eta_{\mathcal{T}}, \eta_{\mathcal{P}}, 1\right)}$ & 0 & 0 \\
\hline & $A_{2}$ & & $\left(A_{1}, A_{2}\right)_{\left(\eta_{\mathcal{T}}, 0,0\right)},\left(B_{1}, B_{2}\right)_{\left(\eta_{\mathcal{T}}, 0,0\right)},\left(E_{1}\right)_{\left(\eta_{\mathcal{T}},-\eta_{\mathcal{P}}, 1\right)},\left(E_{2}\right)_{\left(\eta_{\mathcal{T}},-\eta_{\mathcal{P}}, 1\right)}$ & $\mathbb{Z}^{2} \times \mathbb{Z}_{2}^{2}$ & $\mathbb{Z}^{2}$ \\
\hline & $B_{1}$ & & $\left(A_{1}, B_{1}\right)_{\left(\eta_{\mathcal{T}}, 0,0\right)},\left(A_{2}, B_{1}\right)_{\left(\eta_{\mathcal{T}}, 0,0\right)},\left(E_{1}, E_{2}\right)_{\left(\eta_{\mathcal{T}}, 0,0\right)}$ & $\mathbb{Z}^{3}$ & $\mathbb{Z}^{3}$ \\
\hline & $B_{2}$ & & $\left(A_{1}, B_{1}\right)_{\left(\eta_{\mathcal{T}}, 0,0\right)},\left(A_{2}, B_{1}\right)_{\left(\eta_{\mathcal{T}}, 0,0\right)},\left(E_{1}, E_{2}\right)_{\left(\eta_{\mathcal{T}}, 0,0\right)}$ & $\mathbb{Z}^{3}$ & $\mathbb{Z}^{3}$ \\
\hline
\end{tabular}

by $\sigma_{0} \tau_{1} K$ before the basis change, now reads as $\sigma_{2} \tau_{2} K$. Since the $C_{2 v}$ symmetry enforces that $H$ is of the form $\sigma_{0} H_{\bar{E}}$ [compare with Eq. (26)], the factor $\sigma_{2}$ must be dropped from the representation of $\mathcal{P}$ since it does not affect $H_{\bar{E}}$, and one arrives at the effective particle-hole conjugation operator $\tau_{2} K$ : the effective particle-hole conjugation operator squares to minus one. The time-reversal operation $\mathcal{T}$, which was given by $\sigma_{2} \tau_{0} K$ in the original basis, is unchanged by the basis transformation. Again omitting the factor $\sigma_{2}$, one finds that the effective time-reversal operation is $\tau_{0} K$. It follows that

$$
\mathfrak{K}_{\left(\eta_{\mathcal{T}}, 1, \eta_{\mathcal{C}}\right)}\left[C_{2 v}, A_{1}\right]=\mathfrak{K}_{\left(-\eta_{\mathcal{T}},-1, \eta_{\mathcal{C}}\right)}[0] .
$$

The same result is found if one performs the Wigner test [see Eqs. (27) and (28)].

For $\Theta=r_{+-}$(the " $B_{2}$ " representation) one finds that the representation $u$ of Table III for the normal-state block $h$ gives the representations $U\left(\mathcal{M}_{x}\right)=i \sigma_{1} \tau_{0}$ and $U\left(\mathcal{M}_{y}\right)=i \sigma_{2} \tau_{0}$ for the full $\mathrm{BdG}$ Hamiltonian $H$, which is already in the canonical form (25). It then immediately follows that the effective particle-hole conjugation operation is $\tau_{1} K$ and the effective time-reversal operation is $\tau_{0} K$. For the remaining two cases $r_{-+}$, and $r_{--}$(the " $B_{1}$ " and " $A_{2}$ " representations), one may proceed as described above for the $A_{1}$ representation, by performing a suitable basis transformation, or use an alternative normal-state representation with $u\left(\mathcal{M}_{x}\right)=i \sigma_{2}, u\left(\mathcal{M}_{y}\right)=i \sigma_{3}$ for $B_{1}$ and $u\left(\mathcal{M}_{x}\right)=i \sigma_{3}, u\left(\mathcal{M}_{y}\right)=i \sigma_{1}$ for $A_{2}$, to which the canonical form (25) applies without the need for a basis transformation. Either way, we find that both the effective time-reversal operation and the effective particle-hole conjugation operation square to unity, $W_{\mathcal{T}}=-\eta_{\mathcal{T}}=1$ and $W_{\mathcal{P}}=$ $\eta_{\mathcal{P}}=1$. As a result, we have

$$
\begin{aligned}
\mathfrak{K}_{\left(\eta_{\mathcal{T}}, 1, \eta_{\mathcal{C}}\right)}\left[C_{2 v}, B_{1,2}\right] & =\mathfrak{K}_{\left(\eta_{\mathcal{T}}, 1, \eta_{\mathcal{C}}\right)}\left[C_{2 v}, A_{2}\right] \\
& =\mathfrak{K}_{\left(-\eta_{\mathcal{T}}, 1, \eta_{\mathcal{C}}\right)}[0] .
\end{aligned}
$$

A remark on classifying groups. We close this section by making a few remarks regarding the group structure of topological classification within $K$ theory. Formally, the group structure within $K$ theory is given by the Grothendieck construction [29,73], where the group elements are represented by ordered pairs $\left(\mathrm{H}_{1}, \mathrm{H}_{2}\right)$ of Hermitian matrix-valued functions $H_{1,2}(\mathbf{k})$ of equal dimension. The two pairs $\left(H_{1}, H_{2}\right)$ and 
TABLE IX. Classifying groups $\mathfrak{K}_{W_{\mathcal{P}}(\alpha)}[0]$ and topological invariants $v$ for the tenfold-way classes $\mathrm{D}$ and $\mathrm{C}, \eta=(0, \pm 1,0)$ (third and fourth columns). These depend on the irrep $r_{\alpha}$ through the outcome of the Wigner test $W_{\mathcal{P}}(\alpha)$ only, which is listed in the first column. The second column lists the effective tenfold-way class corresponding to $W_{\mathcal{P}}(\alpha)$. The fifth and sixth columns list a generating Hamiltonian $H_{\text {gen }}$ and a reference Hamiltonian $H_{\text {ref }}$, respectively. The seventh and eighth columns give the representations of particle-hole conjugation $\mathcal{P}$ and the representation $U$ of the elements of the crystalline symmetry group $G$. The dimension of the irreducible representation $r_{\alpha}$ is denoted $d_{\alpha}$.

\begin{tabular}{lccccccc}
\hline \hline$W_{\mathcal{P}}(\alpha)$ & Cartan & $\mathfrak{K}_{\left(0, W_{P}(\alpha), 0\right)}[0]$ & $v$ & $H_{\text {gen }}$ & $H_{\text {ref }}$ & $\mathcal{P}$ \\
\hline 1 & $\mathrm{D}$ & $\mathbb{Z}_{2}$ & $\mathfrak{p}_{\alpha}$ & $-\tau_{3} \otimes 1_{d}$ & $\tau_{3} \otimes 1_{d}$ & $\tau_{1} K$ \\
-1 & $\mathrm{C}$ & 0 & $\mathfrak{N}_{\alpha}$ & & $\tau_{3} \otimes 1_{d}$ & $\tau_{2} K$ & $r_{\alpha} \otimes \tau_{0}$ \\
0 & $\mathrm{~A}$ & $\mathbb{Z}$ & $-\tau_{3} \otimes 1_{d}$ & $\tau_{3} \otimes 1_{d}$ & $\tau_{1,2} K$ & $\left(\begin{array}{c}r_{\alpha} \\
0\end{array}\right.$ \\
\hline \hline
\end{tabular}

$\left(H_{1}^{\prime}, H_{2}^{\prime}\right)$ are topologically equivalent if $H_{1} \oplus H_{2}^{\prime}$ is continuously deformable to $H_{1}^{\prime} \oplus H_{2}$. Loosely speaking, the ordered pair $\left(H_{1}, H_{2}\right)$ represents the "difference" of the two Hamiltonians $H_{1}$ and $H_{2}$.

For gapped phases with dimension $d>0$, a meaningful concept of "topologically nontrivial" Hamiltonians can be obtained by defining a reference atomic-limit Hamitonian $H_{\text {ref }}(\mathbf{k})$ as a "topologically trivial phase." (A precise definition of "atomic-limit Hamiltonians" will be given in the next section.) Such a strategy results in a unique and well-defined topological classification that is independent of the choice of the precise reference Hamiltonian $H_{\text {ref }}(\mathbf{k})$ if one considers Hamiltonians that differ by an atomic-limit Hamiltonian to be in the same topological class. It is this classification principle that underlies the classifying groups $\mathcal{K}_{\eta}[G, \Theta]$ used throughout the remainder of this paper for Hamiltonians of dimension $d \geqslant 1$. On the other hand, for zero-dimensional Hamiltonians, there is no natural choice for the trivial phase and it is important to adhere to the notion that a topological classification classifies pairs of Hamiltonians only. It is this stricter notion of topological classification that is used for the definition of the classifying groups $\mathfrak{K}_{\eta}[G, \Theta]$ of zerodimensional Hamiltonians, which play a key role in the construction of symmetry-based indicators in the next section.

Generators and invariants for the classifying groups $\mathfrak{K}_{\eta}[G, \Theta]$. The classifying groups $\mathfrak{K}_{\eta}[G, \Theta]$ of zerodimensional Hamiltonians with additional point group $G$ and with one-dimensional representation $\Theta$ governing the pairing term $\Theta$ are determined by the effective Cartan class of diagonal blocks corresponding to the irreducible representation $\alpha$ or by pairs or quadruples of such blocks, as discussed above. We tabulate the classification $\mathfrak{K}_{\eta}[0]$, the invariants $v$, generators $H$, and representations $U(g)$ of all symmetry elements $g, \mathcal{T}$, and $\mathcal{P}$ for all cases in Tables IX and $\mathrm{X}$. The zero-dimensional invariants can be given as the number $\mathfrak{N}_{\alpha}$ of negative-energy eigenstates [Kramers pairs in case the block $H_{\alpha}$ is invariant under an antiunitary symmetry $U K$ with $(U K)^{2}=-1$, i.e., in Cartan class AII] with representation $\alpha$ or the Pfaffian invariant $\mathfrak{p}_{\alpha}$ of the block $H_{\alpha}$ belonging to the irreducible representation $r_{\alpha}$. In case there are multiple blocks related by $\mathcal{T}$ or $\mathcal{P}$, we present all equivalent invariants.

For the calculation, the zero-dimensional invariants $v_{\alpha}=$ $\mathfrak{N}_{\alpha}$ or $\mathfrak{p}_{\alpha}$ it may be helpful to use the projector [74] onto a subspace spanned by irreducible representation $\alpha$ :

$$
P_{\alpha}=d_{\alpha}\left\langle\chi_{\alpha}^{*}(g) U(g)\right\rangle_{G},
$$

where $\langle\ldots\rangle_{G}$ denotes the average over all elements $g \in G$ and $\chi_{\alpha}(g)$ is the character. Choosing a basis in which the projector $P_{\alpha}$ is block diagonal, which can be done for all irreps $r_{\alpha}$ simultaneously, although this is not necessary for the calculation to succeed, the projected Hamiltonian $P_{\alpha} H P_{\alpha}$ takes the form $\operatorname{diag}\left(H_{\alpha}, 0_{N-N_{\alpha}}\right)$, and the topological invariant can be computed as

$$
v_{\alpha}(H) \equiv v\left(H_{\alpha}\right)
$$

The Pfaffian invariant $\mathfrak{p}_{\alpha}$ of a subblock $H_{\alpha}$ invariant under an (effective) antiunitary antisymmetry with representation $U$ with $(U K)^{2}=1$ is defined as

$$
(-1)^{\mathfrak{p}_{\alpha}}=\operatorname{sign} \operatorname{Pf}\left(H_{\alpha} U\right) .
$$

\section{SYMMETRY-BASED INDICATORS OF BAND TOPOLOGY}

Whereas a full topological classification of a gapped Hamiltonian in $d$ dimensions, as described by the classifying group $\mathcal{K}_{\eta}[G, \Theta]$, requires the analysis of matrix-valued functions $H(\mathbf{k})$ with the momentum $\mathbf{k}$ taken on the full Brillouin zone, partial information on the topological phase can be already obtained by inspection of the topological class of matrices $H\left(\mathbf{k}_{\mathrm{s}}\right)$ at a discrete set of high-symmetry momenta $\mathbf{k}_{\mathrm{s}}$. Such an approach has been developed by Po et al. for nonsuperconducting insulators [27] (see also Refs. [28,59].) We here present this approach in such a way that it can immediately be generalized to Hamiltonians of Bogoliubovde Gennes type.

We consider a band structure defined by the Hamiltonian $H(\mathbf{k})$, with $\mathbf{k}$ an element of the Brillouin zone of a $d$ dimensional crystal with (discrete) translation invariance and with symmorphic crystalline symmetry described by the point group $G$. In addition, there may be nonspatial symmetries such as time-reversal symmetry, particle-hole antisymmetry, or chiral antisymmetry, which determine the tenfold-way symmetry class. These nonspatial symmetries are characterized by the triple $\eta=\left(\eta_{\mathcal{T}}, \eta_{\mathcal{P}}, \eta_{\mathcal{C}}\right)$, as explained in the previous section. For superconductors, i.e., for Hamiltonians of BdG type, one further needs to specify a one-dimensional representation $\Theta$ of $G$, which characterizes the superconducting phase (see the discussion in Sec. II).

\section{A. Construction of a reference set of high-symmetry momenta}

To define a representative set of high-symmetry momenta, we consider the group $\tilde{G}$ of automorphisms of the Brillouin zone that are induced by elements of $G$ and by the operations $\mathcal{T}$ and $\mathcal{P}$, if present. (The element $g \in G$ induces an automorphism of the Brillouin zone by sending $\mathbf{k} \rightarrow g \mathbf{k}$, whereas 
TABLE X. Classifying groups $\mathfrak{K}_{W_{\mathcal{P}}(\alpha)}[0]$ and topological invariants $v$ for the tenfold-way classes with time-reversal symmetry (third and fourth columns). These depend on the irrep $r_{\alpha}$ through the outcome of the Wigner test $W_{\mathcal{P}}(\alpha)$ only, which is listed in the first column. The second column lists the effective tenfold-way class corresponding to $W_{\mathcal{P}}(\alpha)$. The fifth and sixth columns list a generating Hamiltonian $H_{\text {gen }}$ and a reference Hamiltonian $H_{\text {ref }}$, respectively. The seventh, eighth, and ninth columns give the representations of time reversal $\mathcal{T}$, particle-hole conjugation $\mathcal{P}$, and the representation $U$ of the elements of the crystalline symmetry group $G$. The dimension of the irreducible representation $r_{\alpha}$ is denoted $d_{\alpha}$.

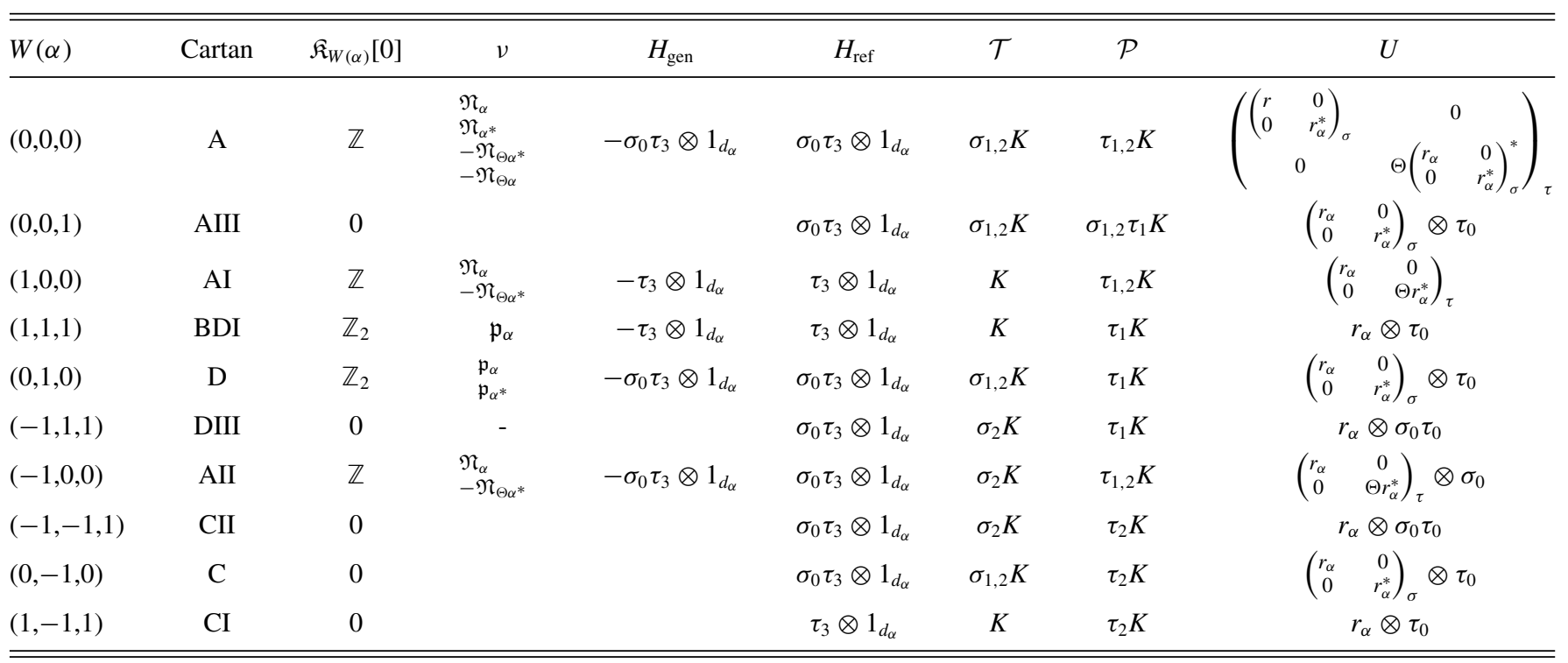

$\mathcal{T}$ and $\mathcal{P}$ send $\mathbf{k}$ to $-\mathbf{k}$, in both cases identifying wave vectors that differ by a reciprocal lattice vector.) For each momentum $\mathbf{k}$ we define the "little group" $\tilde{G}_{\mathbf{k}}$ as the subgroup of elements $\tilde{g} \in \tilde{G}$ such that $\tilde{g} \mathbf{k}=\mathbf{k}$. Two momenta $\mathbf{k}_{1}$ and $\mathbf{k}_{2}$ are considered equivalent for classification purposes if there exists an element $\tilde{g} \in \tilde{G}$ and a continuous path between $\mathbf{k}_{1}$ and $\tilde{g} \mathbf{k}_{2}$ along which the little group $\tilde{G}_{\mathbf{k}}$ does not change. (In particular, this implies that $\mathbf{k}_{1}$ and $\mathbf{k}_{2}$ are equivalent if $\mathbf{k}_{1}=$ $\tilde{g} \mathbf{k}_{2}$ and that equivalent momenta have the same little group.) A set of equivalent momenta is called of "high symmetry" if it does not border to another set of equivalent momenta with a strictly larger little group. The representative set $\left\{\mathbf{k}_{\mathrm{s}}\right\}$ of high-symmetry momenta is then constructed by arbitrarily selecting one momentum from each set of equivalent highsymmetry momenta.

\section{B. Definition of the groups "BS" and "SI"}

To each high-symmetry momentum $\mathbf{k}_{\mathrm{s}}$ we may associate a subgroup $G_{\mathbf{k}_{\mathrm{s}}} \subset G$ of crystalline symmetry operations that leave $\mathbf{k}_{\mathrm{s}}$ invariant and a triple $\eta_{\mathbf{k}_{\mathrm{s}}}=\left(\eta_{\mathcal{T}, \mathbf{k}_{\mathrm{s}}}, \eta_{\mathcal{P}, \mathbf{k}_{\mathrm{s}}}, \eta_{\mathcal{C}}\right)$ that indicate whether $\mathbf{k}_{\mathrm{s}}$ is invariant under the operations $\mathcal{T}$ and $\mathcal{P}$, if present. The group $G_{\mathbf{k}_{\mathrm{s}}}$, the symmetry indices $\eta_{\mathbf{k}_{\mathrm{s}}}$, and the one-dimensional representation $\Theta$ of $G$ (suitably restricted to $G_{\mathbf{k}_{\mathrm{s}}}$ ) determine the symmetry of the Hamiltonian $H\left(\mathbf{k}_{\mathrm{s}}\right)$ at the high-symmetry momentum $\mathbf{k}_{\mathrm{s}}$. The group of "band labels" $\mathrm{BL}$ is defined as the combined set of topological invariants that can be obtained from the topological information at these high-symmetry momenta alone [64]:

$$
\mathrm{BL}_{\eta}[G, \Theta]=\prod_{\mathbf{k}_{\mathrm{s}}} \mathfrak{K}_{\eta_{\mathbf{k s}}}\left[G_{\mathbf{k}_{\mathrm{s}}}, \Theta\right]
$$

The classifying group $\mathfrak{K}_{\eta_{\mathbf{k}}}\left[G_{\mathbf{k}}, \Theta\right]$ describes zero-dimensional topological phases protected by the onsite symmetry group $G_{\mathbf{k}}$ and can be calculated from Eq. (29).

Although this procedure associates a well-defined topological invariant with each Hamiltonian $H(\mathbf{k})$, there are three reasons why $\mathrm{BL}_{\eta}[G, \Theta]$ is different from the classifying group $\mathcal{K}_{\eta}[G, \Theta]$ of topological phases with tenfold-way symmetries $\eta$, point group $G$, and a superconducting phase with symmetry described by $\Theta$ : (i) not every element in BL represents the band labels of a gapped Hamiltonian $H(\mathbf{k})$, (ii) there may exist $d$-dimensional Hamiltonians $H(\mathbf{k})$ without topologically protected anomalous boundary states for which the band labels are nontrivial nevertheless, and (iii) there may be Hamiltonians $H(\mathbf{k})$ with topologically protected anomalous boundary states for which the band labels are trivial.

Problem (i) is addressed by the introduction of "compatibility relations," constraints on the band labels, which follow from the fact that $H(\mathbf{k})$ is not only gapped at the high-symmetry momenta $\mathbf{k}_{\mathrm{s}}$, which is what allows the band labels to be defined in the first place, but also in the remainder of the Brillouin zone $[27,28,56]$. These constraints appear, in the first place, because for a gapped Hamiltonian $H(\mathbf{k})$ any band labels that can be defined for lower-symmetry momenta $\mathbf{k} \notin\left\{\mathbf{k}_{\mathrm{s}}\right\}$ remain well defined and continuous if $\mathbf{k}$ approaches a high-symmetry momentum $\mathbf{k}_{\mathrm{s}}$. Since band labels are essentially zero-dimensional topological invariants (see the discussion above), we call these compatibility relations of "zero-dimensional" ("OD”) type. Po et al. use these "Od compatibility relations" to define the subgroup BS $\subset \mathrm{BL}$ of "topological band labels"

$$
\mathrm{BS}=\left.\mathrm{BL}\right|_{0 \mathrm{D} \text { compatibility relations }} .
$$

We use $B[H(\mathbf{k})]$ to denote the element of BS associated with the gapped Hamiltonian $H(\mathbf{k})$. 
Whereas the group BL of band labels in principle depends on the choice of the set of high-symmetry momenta $\left\{\mathbf{k}_{\mathrm{s}}\right\}$, the group BS is independent of this choice, as long as sufficiently many momenta are included. To see this, we note that the inclusion of additional momenta beyond those appearing in the set $\left\{\mathbf{k}_{\mathrm{s}}\right\}$ of high-symmetry momenta of Sec. IV A adds band labels to BL, but not to BS, as band labels at lowersymmetry momenta are fully determined by the 0D compatibility relations.

Additional constraints on topological band labels of gapped Hamiltonians [compatibility relations of "one-dimensional" ("1D") or "two-dimensional" ("2D") type] may also appear because of the existence of topological invariants on families of higher-dimensional subspaces of the Brillouin zone [29,58], as we discuss below in more detail. Elements in BL or BS that violate the compatibility constraints describe "representation-enforced" gapless phases $[27,58,63]$. Depending on the dimension of the lower-symmetry region in reciprocal space and the type of constraint imposed on it, these representation-enforced gapless phases may have nodal points, nodal lines, or nodal planes.

Problem (ii) can be remedied by passing to the quotient group [27]

$$
\mathrm{SI}=\mathrm{BS} / \mathrm{AI},
$$

the group of "symmetry-based indicators." Here, AI $\subset$ BS is the subgroup generated by the image under $B$ of all Hamiltonians $H(\mathbf{k})$ without boundary signature. A generating set of Hamiltonians without boundary states consists of the "atomiclimit" Hamiltonians, $d$-dimensional Hamiltonians that correspond to the arrangement of zero-dimensional Hamiltonians on a suitably defined lattice. After dividing out AI, a gapped Hamiltonian $H(\mathbf{k})$ is associated with a nontrivial element of SI only if it has topologically protected anomalous boundary states. Po et al. use the notation $X_{\mathrm{BS}}$ to refer to the group of symmetry-based indicators.

There is no general solution to address problem (iii), however. This is why the group SI is said to contain "indicators" of the topology, not a full classification [27].

Although the topological invariants of zero-dimensional Hamiltonians are only defined for pairs of Hamiltonians, the symmetry-based indicators are expressed in terms of the band labels of a general Hamiltonian $H(\mathbf{k})$ with $\mathbf{k}$-independent symmetry representation, without comparing to a reference Hamiltonian.

\section{Construction of the subgroup AI $\subset$ BS}

Following Po et al. [27] and Bradlyn et al. [28], the construction of the subgroup AI proceeds in three steps: (1) One first selects a representative collection $W$ of highsymmetry Wyckoff positions in the unit cell. Hereto, one defines the site-symmetry group $G_{\mathbf{x}}$ for a position $\mathbf{x}$ in the unit cell as the subgroup of $G$ that leaves $\mathbf{x}$ invariant, possibly up to lattice translations, and arranges lattice positions with the same site-symmetry group which are related by a continuous path and/or by an element of $G$ into equivalence classes ("Wyckoff positions"). A representative collection of positions $W$ in the unit cell is then obtained by choosing a position from each equivalence class that does not border on an equivalence class with a larger site-symmetry group. (2) For each position $\mathbf{x} \in W$ the "orbit" of $\mathbf{x}$ is defined as the set $\{g \mathbf{x}+\boldsymbol{t} \mid g \in G\}$ for $\boldsymbol{t}$ in Bravais lattice. The orbit defines a $G$-symmetric Bravais lattice. We label the lattice sites within the unit cell by $O_{\mathbf{x}}=\left\{\mathbf{x}_{\sigma}\right\}_{\sigma=1,2, \ldots}$, with the convention $\mathbf{x}_{1} \equiv \mathbf{x}$. (3) For each Wyckoff position $\mathbf{x}$ and each pair $(\alpha, \Theta)$ of an irreducible representation of the site-symmetry group $G_{\mathbf{x}}$ and the associated one-dimensional representation $\Theta$ describing the symmetry of the superconducting order parameter, we construct a pair of atomic-limit Hamiltonians $H_{\mathrm{x}, \mathrm{ref}}^{(\alpha, \Theta)}$ and $H_{\mathbf{x}, \operatorname{gen}}^{(\alpha, \Theta)}$ by placing the zero-dimensional reference and generating Hamiltonians $H_{\text {ref }}$ and $H_{\text {gen }}$ of $\mathfrak{K}_{\eta}\left[G_{\mathbf{x}}, \Theta\right]$ on the positions $\mathbf{x}_{\sigma}$ of the orbit of $\mathbf{x}$. One verifies that invariance of the zero-dimensional Hamiltonians $H_{\mathbf{x}, \text { ref }}^{(\alpha, \Theta)}$ and $H_{\mathbf{x}, \text { gen }}^{(\alpha, \Theta)}$ under the site-symmetry group $G_{\mathbf{x}}$ ensures that such a procedure yields well-defined $G$-symmetric, translationally invariant, Hamiltonians $H_{\mathbf{x}, \text { ref }}^{(\alpha, \Theta)}(\mathbf{k})$ and $H_{\mathbf{x}, \text { gen }}^{(\alpha, \Theta)}(\mathbf{k})$ on a $d$-dimensional lattice. The subgroup AI $\subset$ BS is generated by the differences $B\left[H_{\mathbf{x}, \text { gen }}^{(\alpha, \Theta)}(\mathbf{k})\right]-B\left[H_{\mathbf{x}, \text { ref }}^{(\alpha, \Theta)}(\mathbf{k})\right]$ of the images of the Hamiltonians obtained in this manner.

The procedure of taking differences of images under $B$ is necessary for the construction of AI because, although the reference Hamiltonians $H_{\text {ref }}$ in Tables IX and X have been chosen such that they map to the trivial element under $B$, the band labels need not be trivial for atomic-limit Hamiltonian obtained by placing copies of $H_{\text {ref }}$ on nontrivial Wyckoff positions. The nontrivial band labels for the reference Hamiltonian have their origin in the $\mathbf{k}$ dependence of the representation of the point group $G$, which is unavoidable if minimal OD superconductors are placed at nontrivial Wyckoff positions. Such a $\mathbf{k}$ dependence of the representation is not compatible with our construction of the topological band labels $B[H(\mathbf{k})]$, which assume a k-independent representation of $G$. [The simplest example where this is the case is a one-dimensional superconductor with inversion symmetry and odd-parity superconducting order (see Sec. V B).]

One can resolve this issue either by adding multiple copies of the "trivial" OD Hamiltonian $H_{\text {ref }}$ at the same Wyckoff position, which eventually allows one to construct a kindependent representation of $G$. We here prefer to take an equivalent, but computationally more efficient, approach, in which we remedy this situation by comparing atomic-limit Hamiltonians with the same number of orbitals [per representation pair $(\alpha, \Theta)]$ at each Wyckoff position only. We construct a "trivial" reference atomic-limit Hamiltonian for this case, and define the band labels of each other atomic-limit Hamiltonian as the difference of the band labels with the reference atomic-limit Hamiltonian. The trivial reference atomiclimit Hamiltonian is chosen such that all Wyckoff positions and all representation pairs $(\alpha, \Theta)$ are represented: It is the direct sum of $0 \mathrm{D}$ reference Hamiltonians $H_{\text {ref }}$ from Tables IX and $\mathrm{X}$ for all pairs $(\alpha, \Theta)$, placed at all Wyckoff positions. Generator atomic-limit Hamiltonians $H_{\mathbf{x}, \operatorname{gen}}^{(\alpha)}(\mathbf{k})$ are obtained by replacing a $0 \mathrm{D}$ reference Hamiltonian in this direct sum by a generator from Tables IX and $\mathrm{X}$ for the orbit $O_{\mathbf{x}}$ and representation pair $(\alpha, \Theta)$ only. Since the map $B$ is additive under the taking of direct sums, taking the difference of the band labels of this generator Hamiltonian and the reference atomic-limit Hamiltonian is the same as taking the difference 
$B\left[H_{\mathbf{x}, \text { gen }}^{(\alpha, \Theta)}(\mathbf{k})\right]-B\left[H_{\mathbf{x}, \text { ref }}^{(\alpha, \Theta)}(\mathbf{k})\right]$ for atomic-limit Hamiltonians involving a single Wyckoff position $\mathbf{x}$ and representation pair $(\alpha, \Theta)$ only, which justifies the procedure outlined in step (iii) above.

Each reference Hamiltonian or generator Hamiltonian is compactly expressed as $[27,28,71]$

$$
H_{\mathbf{x}}^{(\alpha, \Theta)}(\mathbf{k})=\mathbb{1}_{\left|O_{\mathbf{x}}\right| \times\left|O_{\mathbf{x}}\right|} \otimes H_{\mathbf{x}}^{(\alpha, \Theta)},
$$

where $\left|O_{\mathbf{x}}\right|$ is the number of sites in the orbit of $\mathbf{x}$. The representation of $g \in G$ is

$$
\begin{aligned}
{\left[U^{\mathbf{x}, \alpha}(g, \mathbf{k})\right]_{\sigma^{\prime} j, \sigma i}=} & \delta_{\mathbf{x}_{\sigma^{\prime}}, g \mathbf{x}_{\sigma}}^{\prime} e^{-i \mathbf{k} \cdot\left(g \mathbf{x}_{\sigma}-\mathbf{x}_{\sigma^{\prime}}\right)} \\
& \times \frac{z_{g, g_{\sigma}}}{z_{g_{\sigma^{\prime}}, g_{\sigma^{\prime}}} g g_{\sigma}}\left[u_{\mathbf{x}}^{\alpha}\left(g_{\sigma^{\prime}}^{-1} t_{\mathbf{x}_{\sigma^{\prime}}-\mathbf{x}_{\sigma}} g g_{\sigma}\right)\right]_{j i},
\end{aligned}
$$

where the indices $\sigma$ and $\sigma^{\prime}$ label the positions $\mathbf{x}_{\sigma}$ in the orbit of $\mathbf{x}$ and the indices $i$ and $j$ the degree of freedom of $H_{\mathbf{x}}^{(\alpha, \Theta)}$. Further, a choice $\left\{g_{\sigma}\right\}_{\sigma=1, \ldots,\left|O_{\mathbf{x}}\right|}$ from $G$ was made in such a way that $g_{\sigma=1}$ is the identity and $g_{\sigma} \mathbf{x}=\mathbf{x}_{\sigma}$ for $\sigma=2, \ldots,\left|O_{\mathbf{x}}\right|$. Each group element is decomposed as $g=t_{\mathbf{R}} g_{\sigma} h$ with $h \in G_{\mathbf{x}}$, $t_{\mathbf{R}} \in T$, and $\mathbf{R}=g \mathbf{x}_{\sigma}-\mathbf{x}_{\sigma^{\prime}}$. The Kronecker symbol $\delta_{\mathbf{x}_{1}, \mathbf{x}_{2}}^{\prime}$ is nonzero and equal to one only when $\mathbf{x}_{1}=\mathbf{x}_{2}+\mathbf{R}$ for some $\mathbf{R}$ in Bravais lattice. The representation $u_{\alpha}$ is given in Tables IX and $X$.

In this construction it is important that time reversal $\mathcal{T}$ and particle-hole conjugation $\mathcal{P}$ are local operations. If $\mathcal{P}$ is not a local operation, an arrangement of disconnected onsite Hamiltonians can acquire a spurious nonlocality, in spite of the absence of intersite matrix elements.

\section{Compatibility relations}

In the examples that we consider in the next sections, we find that it is sufficient to use compatibility relations for gapped Hermitian matrices $H(\mathbf{k})$ based on local-in-k symmetries only. These compatibility relations may be applied to the entire matrix $H(\mathbf{k})$ or to a diagonal block, if $H(\mathbf{k})$ has a block structure that is preserved throughout a part of the Brillouin zone. The compatibility relations involve topological invariants of $H(\mathbf{k})$ defined using information about $H(\mathbf{k})$ away from the high-symmetry points $\mathbf{k}_{\mathrm{s}}$. Depending on the dimensionality of the subspace required to define these topological invariants, we distinguish between compatibility relations of OD type (which are used to define the group BS of topological band labels) and of 1D or 2D type [64]. We now discuss the construction of $0 \mathrm{D}, 1 \mathrm{D}$, and 2D compatibility relations in detail.

Oa. The number of negative eigenvalues of a Hermitian matrix $H(\mathbf{k})$ with a gapped spectrum does not change if $\mathbf{k}$ is changed continuously. This gives a compatibility constraint if $\mathbf{k}$ can be changed continuously between high-symmetry points $\mathbf{k}_{\mathrm{s}, 1}$ and $\mathbf{k}_{\mathrm{s}, 2}$, while preserving Hermiticity of (a subblock of) the Hamiltonian, and if the number of negative eigenvalues can be related to the band labels at $\mathbf{k}_{\mathrm{s}, 1}$ and $\mathbf{k}_{\mathrm{s}, 2}$.

$O b$. The sign of the Pfaffian of a gapped, antisymmetric matrix $U H(\mathbf{k})$, with $U$ an appropriately chosen unitary operator, does not change if $\mathbf{k}$ is changed continuously. This results in a compatibility constraint for topological band labels if $\mathbf{k}$ can be changed continuously between high-symmetry points $\mathbf{k}_{\mathrm{s}, 1}$ and $\mathbf{k}_{\mathrm{s}, 2}$, while preserving the antisymmetry of (a subblock of) the matrix $U H(\mathbf{k})$, and if the sign of the Pfaffian can be related to the band labels at $\mathbf{k}_{\mathrm{s}, 1}$ and $\mathbf{k}_{\mathrm{s}, 2}$.

1. The topological invariant of a one-dimensional Hamiltonian $H(k)$, obtained by restricting $H(\mathbf{k})$ to a one-dimensional closed contour in reciprocal space, does not change if this contour is continuously deformed. Such a topological invariant can be a winding number, if $H(k)$ has a chiral antisymmetry, but it may also be a $\mathbb{Z}_{2}$ invariant (the first Stiefel-Whitney number $[75,76])$, if $H(k)$ or a diagonal block of it satisfy local-in-k symmetries that place it effectively in tenfold-way classes AI or BDI. This gives an additional compatibility relation if the topological invariant can be related to the topological band labels of $H\left(\mathbf{k}_{\mathrm{s}}\right)$ at high-symmetry points $\mathbf{k}_{\mathrm{s}}$ on the contour and if the contour can be deformed so that it can be made to pass through different sets of high-symmetry momenta, while preserving local-in-k symmetries of $H(\mathbf{k})$. An example of such a 1D compatibility relation is given in the example discussed in Sec. VIID (tenfold-way class CI with inversion symmetry).

2. The topological invariant of a gapped Hermitian matrix $H\left(k_{1}, k_{2}\right)$, which is defined on a two-dimensional plane cutting through the Brillouin zone, does not change if the position of the plane is shifted continuously. The topological invariant is a Chern number if the local-in-k crystalline symmetries are such that $H\left(k_{1}, k_{2}\right)$ or a diagonal block of it are effectively in tenfold-way classes $\mathrm{A}, \mathrm{D}$, or $\mathrm{C}$, or it may be a $\mathbb{Z}_{2}$ invariant (the second Stiefel-Whitney number [75,76]), if $H\left(k_{1}, k_{2}\right)$ is effectively in tenfold-way classes CI or AI. This yields an additional compatibility relation if the topological invariant can be related to the band labels of $H\left(\mathbf{k}_{\mathrm{s}}\right)$ at high-symmetry points $\mathbf{k}_{\mathrm{s}}$ on the plane and if the plane can be shifted continuously, such that it can be made to pass through different sets of highsymmetry momenta while preserving the Hermiticity of (the relevant subblock of) $H(\mathbf{k})$. This " $2 \mathrm{D}$ compatibility relation" is used in Ref. [27] to identify representation-enforced nodal semimetals (see also Refs. [29,58]). It also appears, e.g., in the example discussed in Sec. VII A (tenfold-way class D with inversion symmetry).

Unlike the construction of the groups BS and AI, we are not aware of a method that allows one to implement the compatibility relations based on higher-dimensional topological invariants in an algorithmic way. The "bottleneck" is the relation between the $n$-dimensional topological invariant used to construct the compatibility relation and the topological band labels of $H(\mathbf{k})$ at high-symmetry momenta $\mathbf{k}_{\mathrm{s}}$, which requires knowledge of the full classification of $n$-dimensional crystalline phases. This is not a problem if the symmetrybased indicators are used to determine the topological phase of a given BdG Hamiltonian with a gapped spectrum, but it does affect the use of SI as a proxy for the full boundary classification group $\mathcal{K}$.

Generalizing the definition of BS [see Eq. (42)], we may define the subgroup series

$$
\mathrm{BS}^{(2)} \subset \mathrm{BS}^{(1)} \subset \mathrm{BS}^{(0)} \equiv \mathrm{BS},
$$

where $\mathrm{BS}^{(n)}$ is the subgroup of BL obtained by imposing all compatibility constraints involving topological invariants of dimension $\leqslant n$. Correspondingly, we may define the group 
$\mathrm{SI}^{(n)} \subset \mathrm{SI}$ of symmetry-based indicators as

$$
\mathrm{SI}^{(2)} \subset \mathrm{SI}^{(1)} \subset \mathrm{SI}^{(0)} \equiv \mathrm{SI},
$$

with

$$
\mathrm{SI}^{(n)}=\mathrm{BS}^{(n)} / \mathrm{AI}
$$

and $\mathrm{SI}^{(0)}=$ SI. For the examples we consider, we find that $\mathrm{SI}^{(d-1)}$ contains no gapless phases, implying that the compatibility relations based on local-in-k symmetries are sufficient for these cases.

\section{E. Weak-pairing limit}

In the weak-pairing limit, superconducting order parameter $\Delta$ much smaller than energy scales typical for the normalstate band structure, the band labels $\mathfrak{N}_{\alpha}^{\mathbf{k}_{\mathrm{s}}}$ and $\mathfrak{p}_{\alpha}^{\mathbf{k}_{\mathrm{s}}}$ of the BdG Hamiltonian $H\left(\mathbf{k}_{\mathrm{s}}\right)$ at the high-symmetry momentum $\mathbf{k}_{\mathrm{s}}$ can be expressed in terms of zero-dimensional topological invariants of the normal-state Hamiltonians $h\left(\mathbf{k}_{\mathrm{s}}\right)$ and $h\left(-\mathbf{k}_{\mathrm{s}}\right)$. For $\mathfrak{N}_{\alpha}^{\mathbf{k}_{s}}$ one has $[65,67,68]$

$$
\begin{aligned}
\mathfrak{N}_{\alpha}^{\mathbf{k}_{\mathrm{s}}} & =\left.n_{\alpha}^{\mathbf{k}_{\mathrm{s}}}\right|_{\text {occ }}+\left.n_{\Theta \alpha^{*}}^{-\mathbf{k}_{\mathrm{s}}}\right|_{\text {unocc }} \\
& =\left.n_{\alpha}^{\mathbf{k}_{\mathrm{s}}}\right|_{\text {occ }}-\left.n_{\Theta \alpha^{*}}^{-\mathbf{k}_{\mathrm{s}}}\right|_{\text {occ }}+n_{\Theta \alpha^{*}}^{-\mathbf{k}_{\mathrm{s}}},
\end{aligned}
$$

where $n_{\alpha}^{\mathbf{k}_{\mathrm{s}}},\left.n_{\alpha}^{\mathbf{k}_{\mathrm{s}}}\right|_{\text {occ }}$, and $\left.n_{\alpha}^{\mathbf{k}_{\mathrm{s}}}\right|_{\text {unocc }}$ are the total number, the number of occupied, and the number of unoccupied bands of the subblock $h_{\alpha}\left(\mathbf{k}_{\mathrm{s}}\right)$ of the normal-state Hamiltonian, respectively. Similarly, the Pfaffian invariant $\mathfrak{p}_{\alpha}^{\mathbf{k}_{s}}$ can be expressed as [68]

$$
\mathfrak{p}_{\alpha}^{\mathbf{k}_{\mathrm{s}}}=\left.n_{\alpha}^{\mathbf{k}_{\mathrm{s}}}\right|_{\text {occ }} \bmod 2
$$

\section{EXAMPLES: ONE DIMENSION}

We now discuss symmetry-based indicators for Bogoliubov-de Gennes-type Hamiltonians for a selected set of point groups $G$ and tenfold-way classes.

\section{A. Trivial point group $G=C_{1}$, class $\mathrm{D}$}

Without crystalline symmetries, the Hamiltonian $H(k)$ is particle-hole antisymmetric at both high-symmetry momenta $k=0$ and $\pi$. In the absence of time-reversal symmetry and spin-rotation symmetry (class D), this gives a $\mathbb{Z}_{2}$ classification for $H(0)$ and $H(\pi)$, so that $\mathrm{BL}=\mathbb{Z}_{2}^{2}$. There are no compatibility relations, hence,

$$
\mathrm{BS}=\mathbb{Z}_{2}^{2}
$$

The corresponding topological invariants are $(-1)^{\mathfrak{p}^{(0)}}=$ sign $\operatorname{Pf}\left[H(0) \tau_{1}\right]$ and $(-1)^{\mathfrak{p}^{(\pi)}}=\operatorname{sign} \operatorname{Pf}\left[H(\pi) \tau_{1}\right]$, giving

$$
B[H(k)]=\left\{\mathfrak{p}^{(0)}, \mathfrak{p}^{(\pi)}\right\} .
$$

Without crystalline symmetries there is only one Wyckoff position in a one-dimensional crystal. Placing zero-dimensional Hamiltonians on the generic Wyckoff position, one obtains two topologically different classes of atomic-limit Hamiltonians:

$$
\mathrm{AI}=\mathbb{Z}_{2} .
$$

As a subgroup of BS, AI corresponds to the pairs $\left(\mathfrak{p}^{(0)}, \mathfrak{p}^{(\pi)}\right)$ with $\mathfrak{p}^{(0)}=\mathfrak{p}^{(\pi)}$. The group SI $=\mathrm{BS} / \mathrm{AI}=\mathbb{Z}_{2}$ is identical to the full classification group

$$
\mathrm{SI}_{\mathrm{D}}\left[C_{1}\right]=\mathcal{K}_{\mathrm{D}}\left[C_{1}\right]=\mathbb{Z}_{2},
$$

the nontrivial element of which describes one-dimensional topological superconductors (the "Kitaev chain"). The symmetry-based indicator for the topological superconductor phase is

$$
z_{1}=\sum_{k_{\mathrm{s}}} \mathfrak{p}^{\left(k_{\mathrm{s}}\right)} \bmod 2 .
$$

In the weak-pairing limit, the indicator (54) gives the parity of the number of Fermi level crossings between $k=0$ and $\pi$ [77,78]. The same expression for the full BdG Hamiltonian was obtained in Ref. [68].

\section{B. Inversion symmetry $\boldsymbol{C}_{\boldsymbol{i}}$, class $\mathrm{D}$}

Representation $\Theta=A_{g}$. For a superconducting order parameter that transforms according to the representation $A_{g}$, inversion may be represented as $\rho_{3} \tau_{0}$, the Pauli matrices $\rho_{3}$ and $\tau_{0}$ acting in parity and particle-hole space, respectively (see Sec. II). At the high-symmetry momenta $k_{\mathrm{s}}=(0),(\pi)$, the Hamiltonian $H\left(k_{\mathrm{s}}\right)=\operatorname{diag}\left(H_{+}\left(k_{\mathrm{s}}\right), H_{-}\left(k_{\mathrm{s}}\right)\right)$ is the diagonal sum of blocks acting within the even- and odd-parity subspaces, where the blocks correspond to the irreducible representations $A_{g}$ and $A_{u}$ of $C_{i}$. The preceding discussion of a one-dimensional Hamiltonian $H(k)$ without crystalline symmetries applies to the two blocks separately. In particular, it follows that $\mathrm{BL}=\mathbb{Z}_{2}^{4}$.

To find the compatibility constraints for gapped Hamiltonians, we note that the combination of inversion and particlehole conjugation gives an antiunitary antisymmetry local in momentum space,

$$
H(k)=-\rho_{3} \tau_{1} H(k)^{*} \rho_{3} \tau_{1} .
$$

As a result, $H(k) \rho_{3} \tau_{1}$ is antisymmetric for all $k$, so that sign $\operatorname{Pf}\left[H(k) \rho_{3} \tau_{1}\right]$ is well defined and $k$ independent if $H(k)$ is gapped. Considering that at the high-symmetry momenta $k=0$ and $\pi$ one has sign $\operatorname{Pf}\left[H(k) \rho_{3} \tau_{1}\right]=(-1)^{\mathfrak{p}_{+}^{(k)}+\mathfrak{p}_{-}^{(k)}}$, one finds the compatibility constraint

$$
\mathfrak{p}_{+}^{(0)}+\mathfrak{p}_{-}^{(0)}=\mathfrak{p}_{+}^{(\pi)}+\mathfrak{p}_{-}^{(\pi)} \bmod 2
$$

for gapped Hamiltonians, where we use the subscripts + and - for the representations $A_{g}$ and $A_{u}$, respectively. Using the compatibility relation (55) to eliminate $\mathfrak{p}_{-}^{(\pi)}$ as an independent band label, we find

$$
\mathrm{BS}=\mathbb{Z}_{2}^{3}, \quad B[H(k)]=\left\{\mathfrak{p}_{+}^{(0)}, \mathfrak{p}_{+}^{(\pi)}, \mathfrak{p}_{-}^{(0)}\right\} .
$$

With inversion symmetry there are two inequivalent Wyckoff positions, labeled $x=0$ and $\frac{1}{2}$. A generating set of atomiclimit Hamiltonians is obtained by placing zero-dimensional inversion-symmetric Hamiltonians at each of the Wyckoff positions. Placing a generator of $\mathfrak{K}_{D}[0]$ with topological invariant $\mathfrak{p}=1$ and irreducible representation $A_{g}$ or $A_{u}$ at position $x=0$ we obtain a $k$-independent Hamiltonian for which inversion is represented as by $\tau_{0}$ or $-\tau_{0}$, respectively, and particle-hole conjugation by $\tau_{1} K$. Under the map $B$, these 
Hamiltonians are mapped to $\{1,0,1\}$ and $\{0,1,0\}$ for the $A_{g}$ and $A_{u}$ representations, respectively. Placing a generator of $\mathfrak{K}_{D}[0]$ with topological invariant $\mathfrak{p}=1$ and irreducible representation $A_{g}$ or $A_{u}$ at the Wyckoff position $x=\frac{1}{2}$, one obtains a $k$-independent Hamiltonian for which inversion is represented by $\tau_{0} e^{i k}$ or $-\tau_{0} e^{i k}$ for $A_{g}$ and $A_{u}$, respectively. At $k=\pi$, the irreducible representations are interchanged, so that under $B$ such a Hamiltonian is mapped to $\{1,0,0\}$ and $\{0,1,1\}$ for the irreducible representations $A_{g}$ and $A_{u}$, respectively. The images of these four generating atomic-limit Hamiltonians under $B$ span the whole group $\mathrm{AI}=\mathrm{BS}$. The quotient group

$$
\mathrm{SI}_{\mathrm{D}}\left[C_{i}, A_{g}\right]=0 .
$$

The conclusion that SI contains no nontrivial gapped phases is consistent with the triviality of the classifying group

$$
\mathcal{K}_{\mathrm{D}}\left[C_{i}, A_{g}\right]=0
$$

for this symmetry class [39].

Representation $A_{u}$. For the representation $A_{u}$ the inversion operation for the BdG Hamiltonian is represented as $\rho_{3} \tau_{3}$ (see Sec. II). The Hamiltonian $H(k)=\operatorname{diag}\left(H_{+}(k), H_{-}(k)\right)$ is block diagonal with respect to the eigenvalues \pm of $\rho_{3} \tau_{3}$, corresponding to the fundamental representations $A_{g, u}$ of $C_{i}$. At $k=0$ and $\pi$, the two blocks are minus the particle-hole conjugate of each other. The topological invariants of $H(0)$ and $H(\pi)$ are $\mathfrak{N}_{+}^{(0)}$ and $\mathfrak{N}_{+}^{(\pi)}$, where $\mathfrak{N}_{ \pm}^{(k)}$ is the number of positive eigenvalues of $H_{ \pm}(k)$. (Note that the topological invariants involve the numbers $\mathfrak{N}_{+}^{(k)}$ for $k=0, \pi$ only; particlehole antisymmetry implies that $\mathfrak{N}_{+}^{(k)}=-\mathfrak{N}_{-}^{(k)}$ at $k=0, \pi$.) Accordingly, $\mathrm{BL}=\mathbb{Z}^{2}$.

The combination of inversion and particle-hole conjugation gives an antiunitary antisymmetry of $H(k)$ that is local in momentum space and squares to -1 , thus, $H(k)$ belongs to class $\mathrm{C}$ with trivial classification in zero dimension. We conclude that there are no compatibility constraints for $\mathfrak{N}_{+}^{(0)}$ and $\mathfrak{N}_{+}^{(\pi)}$, so that

$$
\mathrm{BS}=\mathbb{Z}^{2}, \quad B[H(k)]=\left\{\mathfrak{N}_{+}^{(0)}, \mathfrak{N}_{+}^{(\pi)}\right\} .
$$

To construct the subgroup AI $\subset$ BS we place zerodimensional generator Hamiltonians at the Wyckoff positions $x=0$ or $\frac{1}{2}$, which gives the $k$-independent Hamiltonian $H=$ $\operatorname{diag}(-1,1)=-\tau_{3}$, with representation $\tau_{1} K$ for particle-hole conjugation. For the Wyckoff position $x=0$ inversion is represented by $\tau_{3}$, so that the corresponding atomic-limit Hamiltonian maps to the element $\{1,1\}$ of BS. For the Wyckoff position $x=\frac{1}{2}$, inversion is represented by $\tau_{3} e^{i k}$. The $A_{g}$ and $A_{u}$ blocks are interchanged at $k=\pi$ and the corresponding atomic-limit Hamiltonian maps to $\{1,-1\}$, where the -1 originates from the difference with an atomic-limit superconductor obtained by placing the reference Hamiltonian at the same Wyckoff position (see Fig. 1 for a schematic picture). The subgroup $\mathrm{AI}=\mathbb{Z} \times 2 \mathbb{Z}$ thus consists of all elements $\left\{\mathfrak{N}_{+}^{(0)}, \mathfrak{N}_{+}^{(\pi)}\right\}$ for which $\mathfrak{N}_{+}^{(0)}-\mathfrak{N}_{+}^{(\pi)}$ is even. The quotient $\mathrm{SI}=$ $\mathrm{BS} / \mathrm{AI}=\mathbb{Z}_{2}$ contains the symmetry-based indicator

$$
z_{1}=\sum_{k_{\mathrm{s}}} \mathfrak{N}_{+}^{k_{\mathrm{s}}} \bmod 2 .
$$

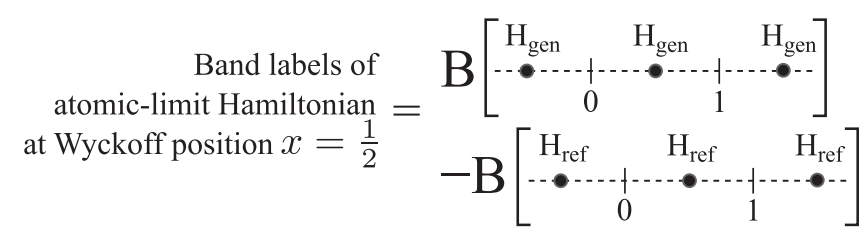

FIG. 1. Topological band labels associated with an atomic-limit superconductor are calculated as the difference of band labels of arrays with the $0 \mathrm{D}$ generator Hamiltonian $H_{\text {gen }}$ and with the $0 \mathrm{D}$ reference Hamiltonian $H_{\text {ref }}$ at Wyckoff position $x$. The figure shows this schematically for the Wyckoff position $x=\frac{1}{2}$.

The conclusion that

$$
\mathrm{SI}_{\mathrm{D}}\left[C_{i}, A_{u}\right]=\mathbb{Z}_{2}
$$

is consistent with the classifying group

$$
\mathcal{K}_{\mathrm{D}}\left[C_{i}, A_{u}\right]=\mathbb{Z}_{2}
$$

for this symmetry class [39], which describes a topological superconductor phase with a single zero-energy Majorana bound state at each end. An example of a nontrivial Hamiltonian in this symmetry class is

$$
H(k)=\operatorname{diag}\left[\tau_{3}(1-m-\cos k)+\tau_{1} \sin k, \tau_{3}\right]
$$

with $0<m<2$.

The weak-pairing limit (49) of the symmetry-based indicator $z_{1}$ agrees with Refs. [66-68]. The symmetry-based indicator $z_{1}$ for the full BdG Hamiltonian agrees with the forms defined in Refs. [67,68].

\section{EXAMPLES: TWO DIMENSIONS}

\section{A. Two mirror symmetries $C_{2 v}$, class D}

The four high-symmetry momenta are $\mathbf{k}_{\mathrm{s}}=(0,0),(0, \pi)$, $(\pi, 0)$, and $(\pi, \pi)$. Each of these four momenta is invariant under the full group $C_{2 v}$ and under particle-hole conjugation, so that the Hamiltonian $H(\mathbf{k})$ has the symmetries corresponding to a zero-dimensional Hamiltonian of tenfold-way class D and with symmetry group $C_{2 v}$. As discussed in Sec. III, such Hamiltonians are topologically trivial if the superconducting phase transforms according to the $A_{1}$ representation of $C_{2 v}$, whereas there is a $\mathbb{Z}_{2}$ invariant $\mathfrak{p}$ for the representations $A_{2}$, $B_{1}$, and $B_{2}$. Hence, we find that

$$
\mathrm{BL}= \begin{cases}0 & \text { for } \Theta=A_{1}, \\ \mathbb{Z}_{2}^{4} & \text { for } \Theta=A_{2}, B_{1}, \text { or } B_{2} .\end{cases}
$$

Representation $\Theta=A_{1}$. For the case $\Theta=A_{1}$, the group of symmetry-based indicators is

$$
\mathrm{SI}_{\mathrm{D}}\left[C_{2 v}, A_{1}\right]=0,
$$

consistent with the triviality

$$
\mathcal{K}_{\mathrm{D}}\left[C_{2 v}, A_{1}\right]=0
$$

of the full classifying group (see Appendix B 4).

Representation $\Theta=B_{2}$. For this representation we have $\mathcal{M}_{x} \mathcal{P}=-\mathcal{P} \mathcal{M}_{x}, \mathcal{M}_{y} \mathcal{P}=\mathcal{P} \mathcal{M}_{y}$, and $\mathcal{M}_{x}^{2}=\mathcal{M}_{y}^{2}=-1$. For $k_{y}=0$ one has the effective "particle-hole antisymmetry" $\mathcal{P} \mathcal{M}_{x}$ with $\left(\mathcal{P} \mathcal{M}_{x}\right)^{2}=1$, which is local in momentum space. The mirror operation $i \mathcal{M}_{y}$ is also local in momentum space 
if $k_{y}=0$, squares to one, and commutes with the effective particle-hole symmetry $\left(\mathcal{P} \mathcal{M}_{x}\right)\left(i \mathcal{M}_{y}\right)=\left(i \mathcal{M}_{y}\right)\left(\mathcal{P} \mathcal{M}_{x}\right)$. We conclude that for each $0<k_{x}<\pi$, the Hamiltonian $H\left(k_{x}, 0\right)$ satisfies an onsite "inversion symmetry" with $\Theta=A_{g}$, so that it has a nontrivial topological classification given by the classifying group $\mathfrak{K}_{\mathrm{D}}\left(C_{i}, A_{g}\right)=\mathbb{Z}_{2}^{2}$. Upon taking the limits $k_{x} \rightarrow 0, \pi$, the classification of $H\left(k_{x}, 0\right)$ for generic $k_{x}$ can be related to the classification at the high-symmetry momenta $k_{x}=0, \pi$, which is given by the topological band labels $\mathfrak{p}^{(0,0)}$ and $\mathfrak{p}^{(\pi, 0)}$. Explicit calculation gives that only the diagonal elements $(\mathfrak{p}, \mathfrak{p})$ of $\mathfrak{K}_{\mathrm{D}}\left(C_{i}, A_{g}\right)=\mathbb{Z}_{2}^{2}$ are allowed, with $\mathfrak{p}=\mathfrak{p}^{(0,0)}=\mathfrak{p}^{(\pi, 0)}$. It follows that one has the compatibility constraint

$$
\mathfrak{p}^{(0,0)}=\mathfrak{p}^{(\pi, 0)} .
$$

In the same way one finds

$$
\mathfrak{p}^{(0, \pi)}=\mathfrak{p}^{(\pi, \pi)} .
$$

One may obtain the same compatibility relations using the explicit representations $\mathcal{P}=\rho_{0} \tau_{1} K, U\left(\mathcal{M}_{x}\right)=i \rho_{1} \tau_{0}$, $U\left(\mathcal{M}_{y}\right)=i \rho_{2} \tau_{0}$ used in Sec. III, so that $H(\mathbf{k})$ satisfies

$$
\begin{aligned}
H\left(k_{x}, k_{y}\right) & =\rho_{1} \tau_{0} H\left(-k_{x}, k_{y}\right) \rho_{1} \tau_{0} \\
& =\rho_{2} \tau_{0} H\left(k_{x},-k_{y}\right) \rho_{2} \tau_{0} \\
& =-\rho_{0} \tau_{1} H\left(-k_{x},-k_{y}\right)^{*} \rho_{0} \tau_{1} .
\end{aligned}
$$

For $k_{y}=0$, these constraints imply that $H\left(k_{x}, 0\right)=$ $-\rho_{1} \tau_{1} H\left(k_{x}, 0\right)^{*} \rho_{1} \tau_{1}=\rho_{2} \tau_{0} H\left(k_{x}, 0\right) \rho_{2} \tau_{0}$, so that we may write $H\left(k_{x}, 0\right)=\rho_{0} h_{0}+\rho_{2} h_{2}$ with $h_{0} \tau_{1}$ and $h_{2} \tau_{1}$ antisymmetric. Further, $h_{0} \pm h_{2}$ is gapped for all $0 \leqslant k_{x} \leqslant \pi$, so that the Pfaffian of $\tau_{1}\left(h_{0} \pm h_{2}\right)$ is nonzero and cannot change sign for $0 \leqslant k_{x} \leqslant \pi$. Since $h_{2}=0$ for $k_{x}=0, \pi$, the compatibility relations (65) and (66) follow immediately. It follows that

$$
\mathrm{BS}=\mathbb{Z}_{2}^{2}, \quad B[H(\mathbf{k})]=\{\mathfrak{p}(\pi, 0), \mathfrak{p}(\pi, \pi)\} .
$$

To construct AI, we place generators at one of the four Wyckoff positions. The Wyckoff positions are $(x, y)=(0,0)$, $\left(0, \frac{1}{2}\right),\left(\frac{1}{2}, 0\right)$, and $\left(\frac{1}{2}, \frac{1}{2}\right)$. From the onsite representations $U\left(\mathcal{M}_{x}\right)=i \rho_{1} \tau_{0}, U\left(\mathcal{M}_{y}\right)=i \rho_{2} \tau_{0}$ we derive the k-dependent representations

$$
\begin{aligned}
& U^{\mathbf{x}}\left(\mathcal{M}_{x}, \mathbf{k}\right)=i \rho_{1} \tau_{0} e^{2 i k_{x} x}, \\
& U^{\mathbf{x}}\left(\mathcal{M}_{y}, \mathbf{k}\right)=i \rho_{2} \tau_{0} e^{2 i k_{y} y}
\end{aligned}
$$

for a zero-dimensional Hamiltonian placed at Wyckoff position $\mathbf{x}=(x, y)$. The $\mathbf{k}$-dependent factors appear because for the Wyckoff positions other than $(0,0)$ the operations $\mathcal{M}_{x}$ and/or $\mathcal{M}_{y}$ correspond to an onsite operation followed by a translation by a lattice vector. As these induced representations differ from the onsite representations by a sign for half of the elements of the symmetry group, which is a change that can be accommodated by a basis transformation, the induced representation is the same at all high-symmetry momenta. After verifying that placing the trivial reference Hamiltonian $H_{\text {ref }}$ at the same Wyckoff positions produces the trivial element in BS, we conclude that the subgroup AI $\subset$ BS consists of the elements with $\mathfrak{p}^{(0,0)}=\mathfrak{p}^{(0, \pi)}$. We conclude that

$$
\mathrm{SI}_{\mathrm{D}}\left[C_{2 v}, B_{2}\right]=\mathbb{Z}_{2},
$$

corresponding to the symmetry-based indicator

$$
z_{1 ; x}=\sum_{\mathbf{k}_{s} \mid k_{s, x}=\pi} \mathfrak{p}^{\mathbf{k}_{\mathrm{s}}} \bmod 2 .
$$

This symmetry-based indicator describes a weak phase of one-dimensional $C_{2 v}$-symmetric topological superconductors in the $y$ direction, stacked in the $x$ direction,

$$
\mathcal{K}_{\mathrm{D}}\left[C_{2 v}, B_{2}\right]=\mathbb{Z}_{2}
$$

(see Appendix B 4). An example of a generator Hamiltonian is

$$
H_{(1 ; x)}=\rho_{0} \tau_{3}\left(1-m-\cos k_{y}\right)+\rho_{1} \tau_{2} \sin k_{y},
$$

with $0<m<2$.

Representation $\Theta=B_{1}$. The discussion for the $B_{1}$ representation is similar to the discussion above. One finds

$$
\begin{aligned}
& \mathrm{SI}_{\mathrm{D}}\left[C_{2 v}, B_{1}\right]=\mathbb{Z}_{2}, \\
& \mathcal{K}_{\mathrm{D}}\left[C_{2 v}, B_{1}\right]=\mathbb{Z}_{2} .
\end{aligned}
$$

The only topologically nontrivial gapped phase is a weak phase of one-dimensional $C_{2 v}$-symmetric topological superconductors in the $x$ direction, stacked in the $y$ direction, for which $z_{1 ; y}=\mathfrak{p}^{(0,0)}+\mathfrak{p}^{(\pi, 0)} \bmod 2$ is the associated symmetry-based indicator.

Representation $\Theta=A_{2}$. The discussion of this representation is easiest if we choose the representation $U\left(\mathcal{M}_{x}\right)=$ $i \rho_{3} \tau_{0}, U\left(\mathcal{M}_{y}\right)=i \rho_{1} \tau_{0}$ (see the discussion in Sec. III). With this choice of representation, one finds that $H\left(k_{x}, k_{y}\right)=$ $h_{0} \rho_{0}$ at the high-symmetry momenta $\left(k_{x}, k_{y}\right)=(0,0),(0, \pi)$, $(\pi, 0)$, and $(\pi, \pi)$. The matrix $\tau_{1} h_{0}$ is antisymmetric and the sign $(-1)^{\mathfrak{p}}$ of its Pfaffian is used as the topological invariant of $H\left(k_{x}, k_{y}\right)$. In contrast to the $B_{1}$ and $B_{2}$ representations, there are no compatibility constraints for the $A_{2}$ representation, hence,

$$
\begin{aligned}
\mathrm{BS} & =\mathbb{Z}_{2}^{4}, \\
B[H(\mathbf{k})] & =\{\mathfrak{p}(0,0), \mathfrak{p}(\pi, 0), \mathfrak{p}(0, \pi), \mathfrak{p}(\pi, \pi)\} .
\end{aligned}
$$

The subgroup AI for the $A_{2}$ representation consists of the elements with $\mathfrak{p}^{(0,0)}=\mathfrak{p}^{(0, \pi)}=\mathfrak{p}^{(\pi, 0)}=\mathfrak{p}^{(\pi, \pi)}$, so that

$$
\mathrm{SI}_{\mathrm{D}}\left[C_{2 v}, A_{2}\right]=\mathbb{Z}_{2}^{3} \text {. }
$$

The classifying group $\mathcal{K}$ for the $A_{2}$ representation is

$$
\mathcal{K}_{\mathrm{D}}\left[C_{2 v}, A_{2}\right]=\mathbb{Z}_{2}^{4}
$$

(see Appendix B 4). Two factors $\mathbb{Z}_{2}$ correspond to weak phases stacked in the $x$ and $y$ directions, with labels " $1 ; x$ " and " $1 ; y$ ", symmetry-based indicators

$$
z_{1 ; j}=\sum_{\mathbf{k}_{\mathbf{s}} \mid k_{\mathrm{s}, j}=\pi} \mathfrak{p}^{\mathbf{k}_{\mathrm{s}}} \bmod 2, \quad j=x, y .
$$

The corresponding generating Hamiltonians are

$$
H_{(1 ; x)}=\rho_{0} \tau_{3}\left(1-m-\cos k_{y}\right)+\rho_{3} \tau_{1} \sin k_{y}
$$

and

$$
H_{(1 ; y)}=\rho_{0} \tau_{3}\left(1-m-\cos k_{x}\right)+\rho_{1} \tau_{1} \sin k_{x},
$$

with $0<m<2$, respectively. The remaining two factors $\mathbb{Z}_{2}$ in the classifying group $\mathcal{K}$ and the single remaining factor $\mathbb{Z}_{2}$ 
TABLE XI. Band labels and symmetry-based indicators for atomic-limit Hamiltonians obtained by placing OD generators at Wyckoff position $\mathbf{x}$ (upper four rows) and for the generators $H_{(1 ; x)}$, $H_{(1 ; y)}, H_{(2,+)}^{\prime}, H_{(2,-)}^{\prime}$ of the weak and second-order phases for the symmetry group $C_{2 v}$ and representation $\Theta=A_{2}$ in two dimensions, class $D$. The weak phases $(1 ; x)$ and $(1 ; y)$ can be constructed from one-dimensional topological superconductors with Majorana bound states, as schematically indicated below (red dots with indicated mirror parity \pm ), stacked in the $x$ or $y$ direction, respectively. A possible boundary signature of the generators of second-order phases consists of Majorana bound states at mirror-symmetric corners with indicated mirror parity. The first column contains the boundarysignature-resolved subgroup sequence for each of the factor groups contributing to the full classification group $\mathcal{K}=\prod_{i} \mathcal{K}_{i}$.

\begin{tabular}{|c|c|c|c|c|c|c|}
\hline \multirow[b]{2}{*}{$\mathcal{K}_{i}^{\prime} \subseteq \mathcal{K}_{i}$} & \multirow[b]{2}{*}{ Phase } & \multicolumn{4}{|c|}{ BS } & \multirow{2}{*}{$\begin{array}{l}\text { SI } \\
\mathbb{Z}_{2}^{3}\end{array}$} \\
\hline & & $\mathfrak{p}^{(0,0)}$ & $\mathfrak{p}^{(\pi, 0)}$ & $\mathfrak{p}^{(0, \pi)}$ & $\mathfrak{p}^{(\pi, \pi)}$ & \\
\hline & $\mathbf{x}=(0,0)$ & 1 & 1 & 1 & 1 & 0 \\
\hline & $\mathbf{x}=\left(\frac{1}{2}, 0\right)$ & 1 & 1 & 1 & 1 & 0 \\
\hline & $\mathbf{x}=\left(0, \frac{1}{2}\right)$ & 1 & 1 & 1 & 1 & 0 \\
\hline & $\mathbf{x}=\left(\frac{1}{2}, \frac{1}{2}\right)$ & 1 & 1 & 1 & 1 & 0 \\
\hline $\mathbb{Z}_{2}$ & $(1 ; x)$ & 1 & 1 & 0 & 0 & $e_{1 ; x}^{(2)}$ \\
\hline $\mathbb{Z}_{2}$ & $(1 ; y)$ & 1 & 0 & 1 & 0 & $e_{1 ; y}^{(2)}$ \\
\hline $\mathbb{Z}_{2} \subseteq \mathbb{Z}_{2}$ & $(2 ;+)^{\prime}$ & 1 & 0 & 0 & 0 & $e_{2}^{(2)}$ \\
\hline $\mathbb{Z}_{2} \subseteq \mathbb{Z}_{2}$ & $(2 ;-)^{\prime}$ & 1 & 0 & 0 & 0 & $e_{2}^{(2}$ \\
\hline
\end{tabular}

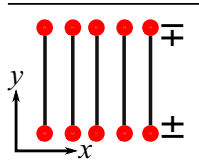

$(1 ; x)$

$z_{1 ; x}=1$

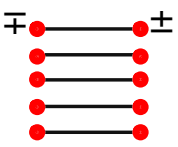

$(1 ; y)$

$z_{1 ; y}=1$

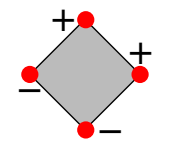

$(2 ;+)^{\prime}$

$z_{2}=1$

$(2 ;-)^{\prime}$
$z_{2}=1$

in SI correspond to strong second-order phases. These have generator Hamiltonians

$$
\begin{aligned}
H_{(2, \pm)}^{\prime}= & \rho_{0} \tau_{3}\left(2-m-\cos k_{x}-\cos k_{y}\right) \\
& \pm \rho_{1} \tau_{1} \sin k_{x}+\rho_{3} \tau_{1} \sin k_{y},
\end{aligned}
$$

with $0<m<2$, but only a single associated symmetry-based indicator

$$
z_{2}=\sum_{\mathbf{k}_{\mathrm{s}}} \mathfrak{p}^{\mathbf{k}_{\mathrm{s}}} \bmod 2 .
$$

As discussed in Appendix B 4, the generator Hamiltonians $H_{(2 ; \pm)}$ describe a second-order phase with a single Majorana corner state at each mirror-symmetric corner, whereas the direct sum $H_{(2 ;+)} \oplus H_{(2 ;-)}$, which is mapped to the trivial element in SI, has pairs of even- and odd-parity Majorana corner states at the corners bisected by one mirror axis, but no corner states at the corners bisected by the other mirror axis.

The results are summarized in Table XI. Following the notation of Ref. [39], we list a full boundary-signatureresolved subgroup series for each singly generated group $\mathcal{K}_{i}$ contributing to the full classification group $\mathcal{K}=\prod_{i} \mathcal{K}_{i}$ that admits topological phases with a higher-order boundary signature. The subgroup $\mathcal{K}_{i}^{\prime} \subset \mathcal{K}_{i}$ is the subset of all topological phases with boundary signature of order larger than one. Weak topological phases in two dimensions, which are essentially stacks of one-dimensional phases, do not have a higher-order boundary signature, so that we do not give a subgroup sequence for factors $\mathcal{K}_{i}$ representing a weak phase.

Table XI also lists the topological band labels for the four Hamiltonians generating the classifying group $\mathcal{K}$, as well as their image in the quotient group SI. Here, we use the symbol $e_{j}^{(n)}$ to denote the $j$ th generator of SI. The superscript $n$ indicates its order, i.e., $n e_{j}^{(n)}=0$.

\section{B. Fourfold rotation symmetry $C_{4}$, class D}

General considerations. There are three nonequivalent high-symmetry momenta $\left\{\mathbf{k}_{\mathrm{s}}\right\}=\{(0,0),(0, \pi),(\pi, \pi)\}$. All three nonequivalent high-symmetry momenta are invariant under particle-hole conjugation. In addition, the momenta $(0,0)$ and $(\pi, \pi)$ are invariant under fourfold rotation, while $(0, \pi)$ is invariant under twofold rotation only.

On a square lattice we can choose the set $W$ of representative Wyckoff positions as $W=\left\{(0,0),\left(\frac{1}{2}, \frac{1}{2}\right),\left(\frac{1}{2}, 0\right)\right\}$. The two Wyckoff positions $\mathbf{x}=(0,0)$ and $\mathbf{x}=\left(\frac{1}{2}, \frac{1}{2}\right)$ are invariant under fourfold rotations; the Wyckoff position $\mathbf{x}=\left(\frac{1}{2}, 0\right)$ is invariant under twofold rotations only. This Wyckoff position has a nontrivial orbit consisting of the positions $\left(\frac{1}{2}, 0\right)$ and $\left(0, \frac{1}{2}\right)$, generated by fourfold rotation. The induced representations $U^{\mathbf{x}, \alpha}(g, \mathbf{k})$ of orbitals located at $\mathbf{x}$ can be written in terms of the onsite (i.e., zero-dimensional) representations $U^{\alpha}(g)$ using

$$
\begin{aligned}
U^{(0,0), \alpha}\left(\mathcal{R}_{\pi / 2}, \mathbf{k}\right) & =U^{\alpha}\left(\mathcal{R}_{\pi / 2}\right), \\
U^{\left(\frac{1}{2}, \frac{1}{2}\right), \alpha}\left(\mathcal{R}_{\pi / 2}, \mathbf{k}\right) & =U^{\alpha}\left(\mathcal{R}_{\pi / 2}\right) e^{i k_{x}}, \\
U^{\left(\frac{1}{2}, 0\right), \alpha}\left(\mathcal{R}_{\pi / 2}, \mathbf{k}\right) & =U^{\alpha}\left(\mathcal{R}_{\pi / 2}\right) \otimes\left(\begin{array}{cc}
0 & 1 \\
e^{i k_{y}} & 0
\end{array}\right),
\end{aligned}
$$

where $\mathcal{R}_{\pi / 2}$ is a rotation by $\pi / 2$ and the matrices for the Wyckoff position $\left(\frac{1}{2}, 0\right)$ act in the space of orbitals contained in its orbit. As in Sec. II, we use the half-integer angular momentum $j$ to denote the irreps $\alpha$ of the rotation symmetry. The angular momentum $j$ is defined modulo 4 for fourfold rotation and modulo 2 for twofold rotation.

The computation of the band labels can be performed with the help of the projector $P_{j}\left(\mathbf{k}_{\mathrm{s}}\right)$ of Eq. (38), which projects the Hamiltonian $H\left(\mathbf{k}_{\mathrm{s}}\right)$ at the high-symmetry momentum $\mathbf{k}_{\mathrm{s}}$ onto its diagonal block with irreducible representation $\alpha=$ $j$. In addition to using Eq. (38) to project on the angular momentum $j$ subspace, a unitary basis transformation $V_{j}$ must be implemented to ensure that $V_{j} P_{j}\left(\mathbf{k}_{\mathrm{s}}\right) V_{j}^{\dagger}$ is block diagonal. (The unitary matrix $V_{j}$ depends on $\mathbf{k}_{\mathrm{s}}$, but we suppress this dependence to keep the notation simple.) In the present case, all irreps are one dimensional and the characters $\chi_{j}$ of Eq. (38) are given by

$$
\chi_{j}\left(\mathcal{R}_{\pi / 2}^{n}\right)=r_{j}^{n}\left(\mathcal{R}_{\pi / 2}\right)=e^{i n j \pi / 2} .
$$

We may then choose the unitary matrix $V \equiv V_{j}$ independent of $j$ by requiring that $V U\left(g, \mathbf{k}_{\mathrm{s}}\right) V^{\dagger}$ be diagonal as in Eq. (19), with $U(g)=\operatorname{diag}_{\alpha}\left[U^{\alpha}\left(g, \mathbf{k}_{\mathrm{s}}\right)\right]$ and $U^{\alpha}\left(g, \mathbf{k}_{\mathrm{s}}\right)$ given in Eq. (78). Indeed, one verifies that with this choice $V P_{j}\left(\mathbf{k}_{\mathrm{s}}\right) V^{\dagger}$ is a diagonal matrix with unit entries for each band $n$ with $\left(V U\left(\mathcal{R}_{\pi / 2}\right) V^{\dagger}\right)_{n n}=r_{j}\left(\mathcal{R}_{\pi / 2}\right)$ and zeros other- 
wise. The transformed Hamiltonian $V H\left(\mathbf{k}_{\mathrm{s}}\right) V^{\dagger}$ is block diagonal and all zero-dimensional invariants can be computed from the block $V P_{j}\left(\mathbf{k}_{\mathrm{s}}\right) H\left(\mathbf{k}_{\mathrm{s}}\right) P_{j}\left(\mathbf{k}_{\mathrm{s}}\right) V^{\dagger}$. In case the block is characterized by a Pfaffian invariant $\mathfrak{p}_{j}\left(\mathbf{k}_{\mathrm{s}}\right)$, the transformed representation of the corresponding antiunitary antisymmetry $V U(\mathcal{P}) V^{T}$ acts within the block such that $(-1)_{j}^{\mathfrak{p}}\left(\mathbf{k}_{\mathrm{s}}\right)=$ sign $\operatorname{Pf}\left(V P_{j}\left(\mathbf{k}_{\mathrm{s}}\right) H\left(\mathbf{k}_{\mathrm{s}}\right) U(\mathcal{P}) V^{T} V P_{j}\left(\mathbf{k}_{\mathrm{s}}\right) V^{\dagger}\right)$ is well defined.

Representations $\Theta=A$ and $B$. These two representations have the same algebraic structure, so that it is sufficient to discuss the case $\Theta=A$ only. (To see this, one considers $i \mathcal{R}_{\pi / 2}$ as the generator of $C_{4}$ for the $B$ representation and verifies that $i \mathcal{R}_{\pi / 2}$ commutes with particle-hole conjugation $\mathcal{P}$.)

The Hamiltonian has fourfold rotation symmetry at the high-symmetry momenta $(0,0)$ and $(\pi, \pi)$. As discussed in Sec. III, for zero-dimensional BdG Hamiltonians with additional symmetry group $C_{4}$ and representation $\Theta=A$, particlehole conjugation pairs the eigensectors corresponding to the irreducible representations $j=\frac{1}{2}$ and $\frac{7}{2}(\bmod 4)$, as well as $j=\frac{3}{2}$ and $\frac{5}{2}(\bmod 4)$. The classifying group $\mathfrak{K}_{\mathrm{D}}\left[C_{4}, A\right]=$ $\mathbb{Z}^{2}$ and the topological invariants are $\mathfrak{N}_{\frac{1}{2}}$ and $\mathfrak{N}_{\frac{5}{2}}$. At the high-symmetry momentum $(0, \pi)$ there is twofold rotation symmetry only. In this case, particle-hole conjugation pairs the angular momenta $j=\frac{1}{2}$ and $\frac{3}{2}(\bmod 2)$ and one has the classification group $\mathfrak{K}_{\mathrm{D}}\left[C_{2}, A\right]=\mathbb{Z}$ and topological invariant $\mathfrak{N}_{\frac{1}{2}}$. A general momentum $\mathbf{k}$ is invariant under $\mathcal{R}_{\pi} \mathcal{P}$ with $\left(\mathcal{R}_{\pi} \mathcal{P}\right)^{2}=-1$ such that the corresponding zero-dimensional Hamiltonians are in class $\mathrm{C}$ with trivial classification. Hence, there are no compatibility relations in this symmetry class. We conclude that

$$
\begin{aligned}
\mathrm{BS} & =\mathbb{Z}^{5}, \\
B[H(\mathbf{k})] & =\left\{\mathfrak{N}_{\frac{1}{2}}^{(0,0)}, \mathfrak{N}_{\frac{5}{2}}^{(0,0)}, \mathfrak{N}_{\frac{1}{2}}^{(\pi, 0)}, \mathfrak{N}_{\frac{1}{2}}^{(\pi, \pi)}, \mathfrak{N}_{\frac{5}{2}}^{(\pi, \pi)}\right\} .
\end{aligned}
$$

The reference and generating Hamiltonians for all Wyckoff positions and (paired) irreducible representations labeled by the angular momentum $j$, as well as the onsite representations of the symmetry group, can be taken directly from Table IX. In particular, we note that

$$
U^{j, 4-j}\left(\mathcal{R}_{\pi / 2}\right)=\operatorname{diag}\left(e^{i j \pi / 2}, e^{i(4-j) \pi / 2}\right)_{\tau},
$$

for $j=\frac{1}{2}$ and $\frac{5}{2}$, where we recall that particle-hole conjugation pairs $j=\frac{1}{2}$ with $j=\frac{7}{2}$ and $j=\frac{3}{2}$ with $j=\frac{5}{2}$. From the induced representation (78) one can then directly compute the band labels of the reference Hamiltonian as well as all generators (see Table S-I in the Supplemental Material) [79]. Upon computing the quotient BS/AI, one finds that the group of symmetry indicators is

$$
\mathrm{SI}_{\mathrm{D}}\left[C_{4}, A\right]=\mathrm{SI}_{\mathrm{D}}\left[C_{4}, B\right]=\mathbb{Z}_{2} \times \mathbb{Z}_{8} .
$$

To interpret the symmetry-based indicators for this representation, we note that this symmetry class has a classifying group [49,50]

$$
\mathcal{K}_{\mathrm{D}}\left[C_{4}, A\right]=\mathcal{K}_{\mathrm{D}}\left[C_{4}, B\right]=\mathbb{Z} \times \mathbb{Z}_{2}^{2} .
$$

The factor $\mathbb{Z}$ in the above group corresponds to a Chern superconductor phase, for which the generating Hamiltonian is

$$
H_{(2)}(\mathbf{k})=\tau_{3}\left(2-m-\cos k_{x}-\cos k_{y}\right)+\tau_{1} \sin k_{x}+\tau_{2} \sin k_{y}
$$

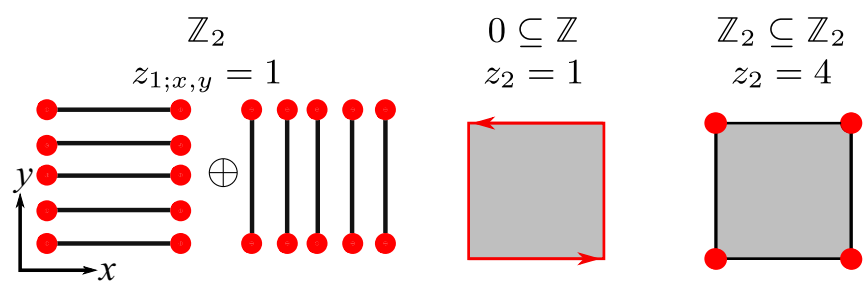

FIG. 2. Topological phases of a two-dimensional superconductor in tenfold-way class $\mathrm{D}$ and with additional $C_{4}$ symmetry with onedimensional representation $\Theta=A, B$. For each boundary signature, the boundary subgroup sequence (top row) and the nonzero symmetry-based indicators for a generator of that phase are given (middle row).

with $0<m<2$ and with (standard) representations $U(\mathcal{P})=$ $\tau_{1}, U\left(\mathcal{R}_{\pi / 2}\right)=e^{-i \pi \tau_{3} / 4}$. Chern superconductors with even Chern numbers $\mathrm{Ch}$ can be deformed such that they have a BdG Hamiltonian with zero pairing potential $\Delta$ and normal part $h$ corresponding to a Chern insulator with Chern number $\mathrm{Ch} / 2$.

Next, one of the factors $\mathbb{Z}_{2}$ of the group (82) corresponds to a weak phase, consisting of two "layers" of $C_{2}$ symmetric Kitaev chains related by a fourfold rotation. [Note that $\mathcal{K}_{\mathrm{D}}\left[C_{2}, A\right]=\mathbb{Z}_{2}$ in one dimension (see Refs. [36,39]).] The generator Hamiltonian is

$$
\begin{aligned}
H_{(1 ; x, y)}(\mathbf{k})= & {\left[\tau_{3}\left(1-m-\cos k_{x}\right)+\tau_{1} \sin k_{x}\right] } \\
& \oplus_{\mu}\left[\tau_{3}\left(1-m-\cos k_{y}\right)-\tau_{2} \sin k_{y}\right]
\end{aligned}
$$

with $0<m<2$, where $\oplus_{\mu}$ denotes that the direct sum acts in "layer space" with Pauli matrices $\mu_{\alpha}$. The representations are $U(\mathcal{P})=\mu_{0} \tau_{1}, U\left(\mathcal{R}_{\pi / 2}\right)=\mu_{1} e^{i \pi \tau_{3} / 4}$. The second factor $\mathbb{Z}_{2}$ corresponds to a second-order phase with four Majorana corner states. The generating Hamiltonian is the direct sum of a twofold symmetric second-order topological superconductor [70] and a copy related by a fourfold rotation

$$
\begin{aligned}
H_{(2)}^{\prime}(\mathbf{k})= & {\left[\rho_{0} \tau_{3}\left(2-m-\cos k_{x}-\cos k_{y}\right)\right.} \\
& \left.+\rho_{3} \tau_{1} \sin k_{x}+\rho_{0} \tau_{2} \sin k_{y}\right] \\
& \oplus_{\mu}\left[\rho_{0} \tau_{3}\left(2-m-\cos k_{x}-\cos k_{y}\right)\right. \\
& \left.+\rho_{0} \tau_{1} \sin k_{x}+\rho_{3} \tau_{2} \sin k_{y}\right]
\end{aligned}
$$

with $0<m<2$, and representations $U(\mathcal{P})=\mu_{0} \rho_{0} \tau_{1}$, $U\left(\mathcal{R}_{\pi / 2}\right)=\mu_{1} \rho_{0} e^{i \pi \tau_{3} / 4}$.

One verifies that the weak phase generates the factor $\mathbb{Z}_{2}$ of SI and has the symmetry-based indicator

$$
z_{1 ; x, y}=\mathfrak{N}_{\frac{1}{2}}^{(\pi, 0)}+\mathfrak{N}_{\frac{1}{2}}^{(\pi, \pi)}+\mathfrak{N}_{\frac{5}{2}}^{(\pi, \pi)} \bmod 2 .
$$

The factor $\mathbb{Z}_{8}$, with the symmetry-based indicator

$$
\begin{aligned}
z_{2}= & -\mathfrak{N}_{\frac{1}{2}}^{(0,0)}+3 \mathfrak{N}_{\frac{5}{2}}^{(0,0)}-2 \mathfrak{N}_{\frac{1}{2}}^{(\pi, 0)} \\
& +3 \mathfrak{N}_{\frac{1}{2}}^{(\pi, \pi)}-\mathfrak{N}_{\frac{5}{2}}^{(\pi, \pi)} \bmod 8,
\end{aligned}
$$

is generated by the band labels of the Chern superconductor. The element " $z_{2}=4$ " of the factor $\mathbb{Z}_{8}$ of SI is ambiguous, as it corresponds to the second-order phase or the Chern superconductor with four Majorana modes (see Fig. 2). These 
classification results and symmetry-based indicators agree with the results from Refs. [49,50].

Representations $\Theta={ }^{1} E$ and ${ }^{2} E$. Here, we consider the case $\Theta={ }^{1} E$ only and note that the case $\Theta={ }^{2} E$ is analogous. In Sec. III we found that the particle-hole conjugation $\mathcal{P}$ with $\Theta={ }^{1} E$ relates the eigensectors with angular momentum $j=\frac{3}{2}$ and $\frac{7}{2}$, while $\mathcal{P}$ acts within the $j=\frac{1}{2}$ and $\frac{5}{2}$ eigensectors. The eigensectors $j=\frac{1}{2}$ and $\frac{3}{2}$ of twofold rotation symmetry are left invariant by particle-hole conjugation. From Table $\mathrm{V}$ we conclude that at each of the highsymmetry momenta $(0,0)$ and $(\pi, \pi)$ with fourfold rotation symmetry we obtain a $\mathfrak{K}_{\mathrm{D}}\left[C_{4},{ }^{1} E\right]=\mathbb{Z} \times \mathbb{Z}_{2}^{2}$ classification of the band labels, with invariants $\mathfrak{N}_{\frac{3}{2}}, \mathfrak{p}_{\frac{1}{2}}$, and $\mathfrak{p}_{\frac{5}{2}}$, whereas for the momentum $(0, \pi)$ with twofold rotation symmetry the classifying group is $\mathfrak{K}_{\mathrm{D}}\left[C_{2}, B\right]=\mathbb{Z}_{2}^{2}$, with invariants $\mathfrak{p}_{\frac{1}{2}}$ and $\mathfrak{p}_{\frac{3}{2}}$. It follows that $\mathrm{BL}=\mathbb{Z}^{2} \times \mathbb{Z}_{2}^{6}$.

The combination of twofold rotation and particle-hole conjugation provides an antiunitary antisymmetry operation that is local in momentum space and squares to one. For gapped Hamiltonians, the quantity $(-1)^{\mathfrak{p}\left(\mathcal{R}_{\pi} \mathcal{P}\right)}=$ sign $\operatorname{Pf}\left[H(\mathbf{k}) U\left(\mathcal{R}_{\pi} \mathcal{P}\right)\right]$ is well defined and constant throughout the Brillouin zone, from which one derives the (0D) compatibility constraints

$$
\begin{aligned}
\mathfrak{p}_{\frac{1}{2}}^{(0, \pi)}+\mathfrak{p}_{\frac{3}{2}}^{(0, \pi)} & =\mathfrak{p}_{\frac{1}{2}}^{(0,0)}+\mathfrak{p}_{\frac{5}{2}}^{(0,0)}+\mathfrak{N}_{\frac{3}{2}}^{(0,0)} \\
& =\mathfrak{p}_{\frac{1}{2}}^{(\pi, \pi)}+\mathfrak{p}_{\frac{5}{2}}^{(\pi, \pi)}+\mathfrak{N}_{\frac{3}{2}}^{(\pi, \pi)} \bmod 2 .
\end{aligned}
$$

We therefore conclude that

$$
\begin{aligned}
\mathrm{BS} & =\mathbb{Z}^{2} \times \mathbb{Z}_{2}^{4}, \\
B[H(\mathbf{k})] & =\left\{\mathfrak{p}_{\frac{1}{2}}^{(0,0)}, \mathfrak{p}_{\frac{5}{2}}^{(0,0)}, \mathfrak{N}_{\frac{3}{2}}^{(0,0)}, \mathfrak{p}_{\frac{3}{2}}^{(\pi, 0)}, \mathfrak{p}_{\frac{5}{2}}^{(\pi, \pi)}, \mathfrak{N}_{\frac{3}{2}}^{(\pi, \pi)}\right\} .
\end{aligned}
$$

As before, we take the onsite reference Hamiltonian and generating Hamiltonians as well as the onsite representations from Table IX. For the angular momenta $j=\frac{3}{2}$ and $\frac{7}{2}$, which are paired by particle-hole conjugation, one has the representation

$$
U^{\frac{3}{2}, \frac{7}{2}}\left(\mathcal{R}_{\pi / 2}\right)=\operatorname{diag}\left(e^{3 \pi i / 4}, e^{7 \pi i / 4}\right)_{\tau} .
$$

With the help of the induced representation (78) the band labels of the atomic-limit Hamiltonians then easily follow (see Table S-II in the Supplementary Material) [79]. By taking the quotient group, one arrives at

$$
\mathrm{SI}_{\mathrm{D}}\left[C_{4},{ }^{1} E\right]=\mathrm{SI}_{\mathrm{D}}\left[C_{4},{ }^{2} E\right]=\mathbb{Z}_{4} \text {. }
$$

The group is generated by a Chern superconductor with Chern number 2 ,

$$
\begin{aligned}
H_{(2)}(\mathbf{k})= & \rho_{0} \tau_{3}\left(2-m-\cos k_{x}-\cos k_{y}\right) \\
& +\rho_{1} \tau_{2} \sin k_{x}+\rho_{1} \tau_{1} \sin k_{y},
\end{aligned}
$$

with $0<m<2$, and representations

$$
U(\mathcal{P})=\rho_{0} \tau_{1}, \quad U\left(\mathcal{R}_{\pi / 2}\right)=\tau_{3} e^{-i \pi / 4} \oplus_{\rho} \tau_{0} e^{i \pi / 4} .
$$

The symmetry-based indicator is

$$
\begin{aligned}
z_{2}= & -\mathfrak{N}_{\frac{3}{2}}^{(0,0)}-\mathfrak{N}_{\frac{3}{2}}^{(\pi, \pi)}+2 \mathfrak{p}_{\frac{5}{2}}^{(0,0)} \\
& +2 \mathfrak{p}_{\frac{3}{2}}^{(\pi, 0)}+2 \mathfrak{p}_{\frac{5}{2}}^{(\pi, \pi)} \bmod 4 .
\end{aligned}
$$

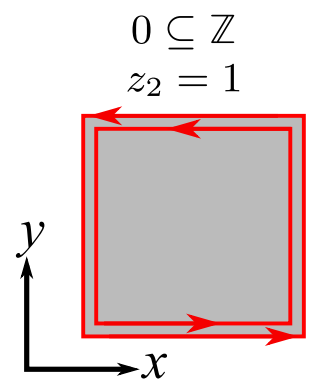

FIG. 3. The only topological phase with nontrivial boundary signature for a two-dimensional superconductor in tenfold-way class $\mathrm{D}$ and with additional $C_{4}$ symmetry with one-dimensional representation $\Theta=\Theta={ }^{1,2} E$ is a Chern superconductor with an even number of chiral Majorana boundary modes. The boundary subgroup sequence for this phase is given in the top row; the symmetry-based indicator for a generator of the phase is given in the middle row.

A Hamiltonian with Chern number 1 is not compatible with the constraints given by the algebraic relations between the representations in this symmetry class.

For comparison, we note that the classifying group is

$$
\mathcal{K}_{\mathrm{D}}\left[C_{4},{ }^{1} E\right]=\mathcal{K}_{\mathrm{D}}\left[C_{4},{ }^{2} E\right]=\mathbb{Z},
$$

which is generated by the even-Chern-number superconductors (see Fig. 3). There are no weak or second-order phases in this symmetry class. This observation is compatible with the absence of weak or second-order phases for a twofold rotation symmetry with $B$ pairing symmetry $[36,39,70]$. We note that the Chern superconductor with even Chern number $\mathrm{Ch}$ is topologically equivalent to a BdG Hamiltonian (1) with zero pairing potential, corresponding to a nonsuperconducting Chern insulator with the Chern number $\mathrm{Ch} / 2$.

\section{EXAMPLES: THREE DIMENSIONS}

\section{A. Inversion symmetry, class D}

There are eight high-symmetry momenta $\mathbf{k}_{\mathrm{s}}$ with $k_{\mathrm{s}, x}$, $k_{\mathrm{s}, y}, k_{\mathrm{s}, z} \in\{0, \pi\}$. With the classifying group $\mathfrak{K}_{\eta}\left[C_{i}, A_{g}\right]=$ $\mathfrak{K}_{\eta}[0]^{2}=\mathbb{Z}_{2}^{2}$ and $\mathfrak{K}_{\eta}\left[C_{i}, A_{u}\right]=\mathfrak{K}_{(0,0,0)}[0]=\mathbb{Z}$ the group of the band labels is

$$
\mathrm{BL}= \begin{cases}\mathbb{Z}_{2}^{16} & \text { for } \Theta=A_{g}, \\ \mathbb{Z}^{8} & \text { for } \Theta=A_{u} .\end{cases}
$$

The band labels are given by the sets of topological invariants $\left\{\mathfrak{p}_{ \pm}\left(\mathbf{k}_{\mathrm{s}}\right)\right\}$ or $\left\{\mathfrak{N}_{+}\left(\mathbf{k}_{\mathrm{s}}\right)\right\}$ for all high-symmetry momenta $\mathbf{k}_{\mathrm{s}}$ for $\Theta=A_{g}$ and $A_{u}$, respectively.

In an inversion-symmetric cubic lattice the set of representative Wyckoff positions $W$ consists of elements $(x, y, z)$ with $x, y, z \in\left\{0, \frac{1}{2}\right\}$. The induced representation $U^{\mathbf{x}, \alpha}(\mathcal{I}, \mathbf{k})$ can be written in terms of the onsite representations $U^{\alpha}(\mathcal{I})$ as

$$
U^{\mathbf{x}, \alpha}(\mathcal{I}, \mathbf{k})=U^{\alpha}(\mathcal{I}) e^{2 i \mathbf{k} \cdot \mathbf{x}} .
$$

Representation $\Theta=A_{g}$. The Hamiltonian $H(\mathbf{k})$ satisfies the antiunitary antisymmetry $\mathcal{I} P$ with $(\mathcal{I} P)^{2}=1$, which is local in momentum space. As a result, the quantity $(-1)^{\mathfrak{p}(\mathcal{I} P)}=$ sign $\operatorname{Pf}[H(\mathbf{k}) U(\mathcal{I} P)]$ is well defined and constant throughout the Brillouin zone, which gives the compatibility relations

$$
\mathfrak{p}_{+}^{\mathbf{k}_{\mathrm{s}}}+\mathfrak{p}_{-}^{\mathbf{k}_{\mathrm{s}}}=\mathfrak{p}_{+}^{\mathbf{0}}+\mathfrak{p}_{-}^{\mathbf{0}} \bmod 2
$$


for the high-symmetry momenta $\mathbf{k}_{\mathrm{s}}$. It follows that

$$
\begin{aligned}
\mathrm{BS} & =\mathbb{Z}_{2}^{9}, \\
B[H(\mathbf{k})] & =\left\{\mathfrak{p}_{+}^{(0,0,0)}, \mathfrak{p}_{-}^{(0,0,0)}, \mathfrak{p}_{+}^{(\pi, 0,0)}, \mathfrak{p}_{+}^{(0, \pi, 0)}, \mathfrak{p}_{+}^{(\pi, \pi, 0)},\right. \\
& \left.\mathfrak{p}_{+}^{(0,0, \pi)}, \mathfrak{p}_{+}^{(\pi, 0, \pi)}, \mathfrak{p}_{+}^{(0, \pi, \pi)}, \mathfrak{p}_{+}^{(\pi, \pi, \pi)}\right\} .
\end{aligned}
$$

Further, for a gapped Hamiltonian $H(\mathbf{k})$, a Chern number $\mathrm{Ch}_{i}$ can be defined on planes with fixed $k_{i}$ with $i=x, y$, or $z$. For the representation $\Theta=A_{g}$ the combination of particlehole and inversion symmetry allows for even Chern numbers $\mathrm{Ch}_{i}$ only. At high-symmetry planes with $k_{i}=0$ or $\pi$, the Chern number $\mathrm{Ch}_{i}$ is related to the band labels as

$$
\begin{aligned}
\mathrm{Ch}_{i} & =2\left(\sum_{\mathbf{k}_{\mathrm{s}}||_{\mathrm{s}, i}=0} \mathfrak{p}_{+}^{\mathbf{k}_{\mathrm{s}}}\right) \bmod 4 \\
& =2\left(\sum_{\mathbf{k}_{\mathrm{s}} \mid \mathrm{k}_{\mathrm{s}, i=\pi}} \mathfrak{p}_{+}^{\mathbf{k}_{\mathrm{s}}}\right) \bmod 4,
\end{aligned}
$$

where we used Eq. (96) to arbitrarily select the invariant $\mathfrak{p}_{+}^{\mathbf{k}_{s}}$ in the even-inversion-parity subspace. Imposing this $2 \mathrm{D}$ compatibility relation further reduces the group of band labels to $\mathrm{BS}^{(2)}=\mathbb{Z}_{2}^{8}$, the independent band labels (97) with the exception of $\mathfrak{p}_{+}^{(\pi, \pi, \pi)}$.

The band labels of atomic insulators, which span the subgroup $\mathrm{AI} \subset \mathrm{BS}$, can be directly computed using the induced representation (95) (see Table S-IV of the Supplementaly Material [79]. Computing the quotient, we find that

$$
\mathrm{SI}_{\mathrm{D}}\left[C_{i}, A_{g}\right]=\mathbb{Z}_{2}^{4}, \quad \mathrm{SI}_{\mathrm{D}}^{(2)}\left[C_{i}, A_{g}\right]=\mathbb{Z}_{2}^{3},
$$

where the four generators of SI are related to the symmetrybased indicators

$$
\begin{aligned}
z_{2 ; i} & =\sum_{\mathbf{k}_{\mathrm{s}} \mid k_{\mathrm{s}, i}=\pi} \mathfrak{p}_{+}^{\mathbf{k}_{\mathrm{s}}} \bmod 2, \quad i=x, y, z \\
z_{3} & =\sum_{\mathbf{k}_{\mathrm{s}}} \mathfrak{p}_{+}^{\mathbf{k}_{\mathrm{s}}} \bmod 2 .
\end{aligned}
$$

(The three generators of $\mathrm{SI}^{(2)}$ have symmetry-based indicators $z_{2 ; i}, i=x, y, z$.) The $\mathbb{Z}_{2}$ indicator $z_{3}$ corresponds to a representation-enforced nodal superconductor with different Chern numbers at parallel planes in the Brillouin zone [see Eq. (98)], and a nodal point at a generic position in the Brillouin zone. The three $\mathbb{Z}_{2}$ indicators $z_{2 ; i}, i=x, y, z$, correspond to weak Chern superconductors with even Chern number in stacking planes (see Fig. 4). Generator Hamiltonians are

$$
\begin{aligned}
H_{(2 ; l)}(\mathbf{k})= & \rho_{0} \tau_{3}\left(2-m-\cos k_{i}-\cos k_{j}\right) \\
& +\rho_{1} \tau_{1} \sin k_{i}+\rho_{1} \tau_{2} \sin k_{j},
\end{aligned}
$$

with $\left(k_{i}, k_{j}\right)=\left(k_{y}, k_{z}\right),\left(k_{z}, k_{x}\right)$, or $\left(k_{x}, k_{y}\right)$ for $l=x, y$, or $z$, respectively. For all three cases, the representations are

$$
U(\mathcal{P})=\rho_{0} \tau_{1}, \quad U(\mathcal{I})=\rho_{3} \tau_{0} .
$$

For comparison, we note that this symmetry class has a classifying group

$$
\mathcal{K}_{\mathrm{D}}\left[C_{i}, A_{g}\right]=\mathbb{Z}^{3}
$$
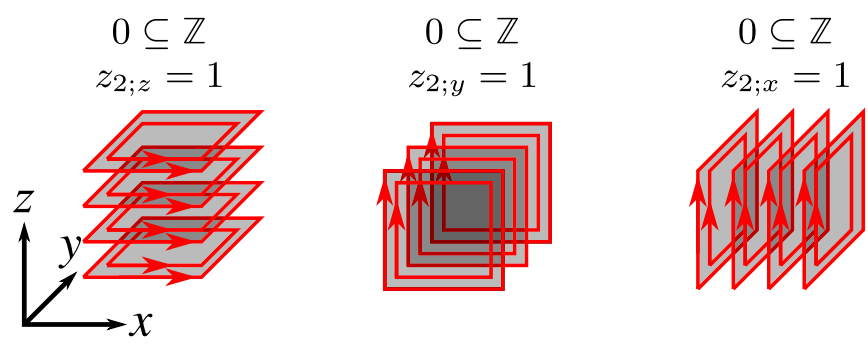

FIG. 4. Topological phases of a three-dimensional superconductor in tenfold-way class $\mathrm{D}$ and with additional $C_{i}$ symmetry with one-dimensional representation $\Theta=A_{g}$. For each boundary signature, the boundary subgroup sequence (top row) and the nonzero symmetry-based indicators for a generator of that phase are given (middle row).

which contains the weak phases with even Chern numbers in the three stacking planes. Generators for these weak phases are shown schematically in Fig. 4. Since each factor $\mathbb{Z}$ in $\mathcal{K}$ describes a weak topological phase, which is obtained by stacking two-dimensional superconductors, only secondorder boundary signatures are allowed in principle, which is why the subgroup sequences listed in Fig. 4 contain one subgroup only. The three weak Chern superconductors with even Chern numbers $\mathrm{Ch}$ per layer can be continuously deformed to normal-state weak Chern insulators with vanishing superconducting correlations and Chern number $\mathrm{Ch} / 2$ per layer.

For comparison, we note that Ref. [67] found no symmetry-based indicators due to the absence of Pfaffian band labels in their construction.

Representation $\Theta=A_{u}$. For the case of the $A_{u}$ representation, there are no compatibility relations of $0 \mathrm{D}$ type, so that

$$
\mathrm{BS}_{\mathrm{D}}\left[C_{i}, A_{u}\right]=\mathrm{BL}_{\mathrm{D}}\left[C_{i}, A_{u}\right]=\mathbb{Z}^{8} .
$$

There is a compatibility relation of $2 \mathrm{D}$ type, however, which follows from the existence of Chern numbers $\mathrm{Ch}_{i}$ along planes at constant $k_{i}, i=x, y, z$ [29]:

$$
\begin{aligned}
\mathrm{Ch}_{i} & =\sum_{\mathbf{k}_{\mathrm{s}} \mid k_{\mathrm{s}, i}=0} \mathfrak{N}_{+}^{\mathbf{k}_{\mathrm{s}}} \\
& =\sum_{\mathbf{k}_{\mathrm{s}} \mid k_{\mathrm{s}, i}=\pi} \mathfrak{N}_{+}^{\mathbf{k}_{\mathrm{s}}} \bmod 2,
\end{aligned}
$$

with $i=x, y, z$. Computing the quotient $\mathrm{SI}=\mathrm{BS} / \mathrm{AI}$, we find that the group of symmetry-based indicators is

$$
\mathrm{SI}_{\mathrm{D}}\left[C_{i}, A_{u}\right]=\mathbb{Z}_{2}^{3} \times \mathbb{Z}_{4}^{3} \times \mathbb{Z}_{8}
$$

Here, the three factors $\mathbb{Z}_{2}$ with symmetry-based indicators

$$
z_{1 ; i, j}=\sum_{\mathbf{k}_{\mathrm{s}} \mid k_{\mathrm{s}, i}=k_{\mathrm{s}, j}=\pi} \mathfrak{N}_{+}^{\mathbf{k}_{\mathrm{s}}} \bmod 2,
$$

for $(i, j)=(x, y),(z, x)$, and $(y, z)$, correspond to weak phases consisting of stacks of one-dimensional topological superconductors (see Fig. 5). Generator Hamiltonians are

$$
H_{(1 ; x, y)}(\mathbf{k})=\tau_{3}\left(1-m-\cos k_{z}\right)+\tau_{1} \sin k_{z}, \quad \text { cycl }
$$



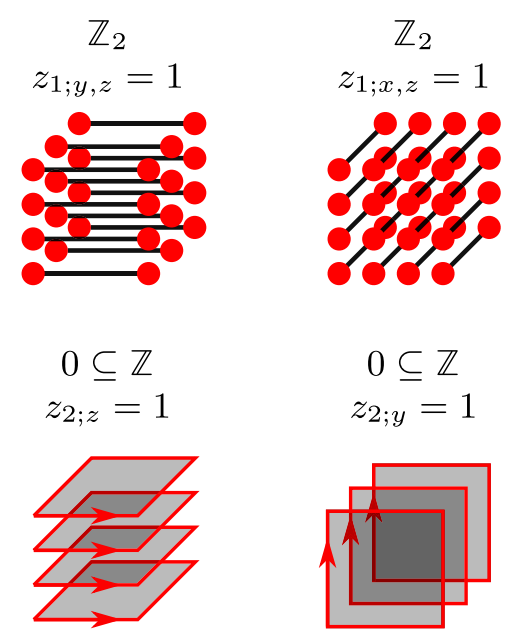

$$
\begin{gathered}
0 \subseteq \mathbb{Z} \\
z_{2 ; y}=1
\end{gathered}
$$
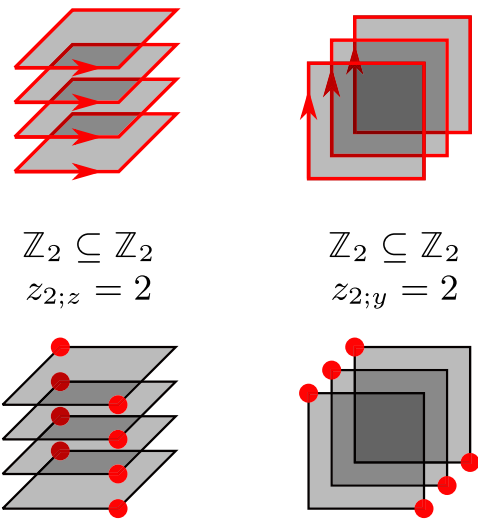

$$
\mathbb{Z}_{2} \subseteq \mathbb{Z}_{2}
$$$$
z_{2 ; y}=2
$$

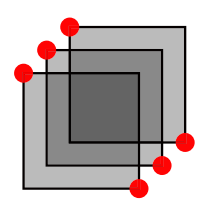

$\mathbb{Z}_{2} \subseteq \mathbb{Z}_{4} \subseteq \mathbb{Z}_{4}$
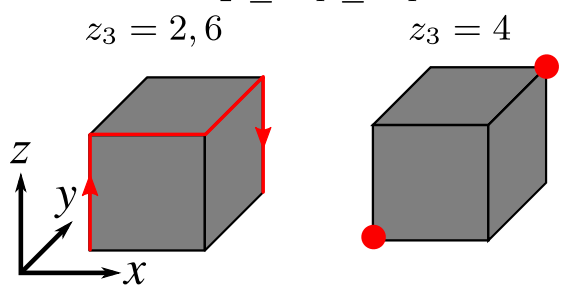

FIG. 5. Topological phases of a three-dimensional superconductor in tenfold-way class $\mathrm{D}$, with additional $C_{i}$ symmetry and onedimensional representation $\Theta=A_{u}$. For each boundary signature, the subgroup sequence and the symmetry-based indicators of the generators of that phase are given. The third-order topological superconductor with $z_{3}=4$ can be constructed as the direct sum of two second-order topological superconductors with $z_{3}=2$.

with the representations

$$
U(\mathcal{P})=\tau_{1}, \quad U(\mathcal{I})=\tau_{3} .
$$

The three factors $\mathbb{Z}_{4}$, which have indicators

$$
z_{2 ; l}=-\sum_{\mathbf{k}_{\mathrm{s}} \mid k_{\mathrm{s}, l}=\pi} \mathfrak{N}_{+}^{\mathbf{k}_{\mathrm{s}}}(-1)^{\left(k_{\mathrm{s}, x}+k_{\mathrm{s}, y}+k_{\mathrm{s},}\right) / \pi} \bmod 4,
$$

for $l=x, y, z$, correspond to weak Chern superconductors (see Fig. 5). Generator Hamiltonians are

$$
\begin{aligned}
H_{(2, x)}(\mathbf{k})= & \tau_{3}\left(2-m-\cos k_{y}-\cos k_{z}\right) \\
& +\tau_{1} \sin k_{y}+\tau_{2} \sin k_{z}, \text { cycl }
\end{aligned}
$$

with $0<m<2$, and the same representations as above. Similar to previous examples, we find that the weak evenChern-number superconductors are continuously deformable to weak normal-state Chern insulators. The symmetry-based indicators $z_{2, l}=2$ are ambiguous as they may also correspond to a weak second-order topological superconductor,

$$
\begin{aligned}
H_{(2, x)}^{\prime}(\mathbf{k})= & \rho_{0} \tau_{3}\left(2-m-\cos k_{y}-\cos k_{z}\right) \\
& +\rho_{3} \tau_{1} \sin k_{y}+\rho_{0} \tau_{2} \sin k_{z}, \text { cycl }
\end{aligned}
$$

with $0<m<2$, and the representations

$$
U(\mathcal{P})=\rho_{0} \tau_{1}, \quad U(\mathcal{I})=\rho_{0} \tau_{3} .
$$

Finally, the factor $\mathbb{Z}_{8}$ with indicator

$$
z_{3}=\sum_{\mathbf{k}_{\mathrm{s}}} \mathfrak{N}_{+}^{\mathbf{k}_{\mathrm{s}}}(-1)^{\left(k_{\mathrm{s}, x}+k_{s, y}+k_{\mathrm{s}, z}\right) / \pi} \bmod 8
$$

is generated by a representation-enforced nodal superconductor with different Chern number at parallel planes. The direct sum of two representation enforced-nodal superconductors may produce a strong second-order topological superconductor with chiral Majorana hinge states and generator Hamiltonian

$$
\begin{aligned}
H_{(3)}^{\prime}(\mathbf{k})= & \rho_{3} \tau_{3}\left(3-m-\cos k_{x}-\cos k_{y}-\cos k_{z}\right) \\
& +\rho_{0} \tau_{1} \sin k_{x}+\rho_{0} \tau_{2} \sin k_{y}+\rho_{2} \tau_{3} \sin k_{z}
\end{aligned}
$$

with $0<m<2$, and with representations

$$
U(\mathcal{P})=\rho_{0} \tau_{1}, \quad U(\mathcal{I})=\rho_{3} \tau_{3} .
$$

The direct sum of two strong second-order topological superconductors in this symmetry class, corresponding to $z_{3}=4$, generates a third-order topological superconductor $[25,39]$. This identifies $z_{3}=2$ and 6 as indicators of strong secondorder phases, whereas $z_{3}=4$ indicates a third-order phase (see Fig. 5).

For comparison, we note that the classifying group for this case is

$$
\mathcal{K}_{\mathrm{D}}\left[C_{i}, A_{u}\right]=\mathbb{Z}_{2}^{6} \times \mathbb{Z}_{4} \times \mathbb{Z}^{3},
$$

where three factors $\mathbb{Z}_{2}$ correspond to weak phases consisting of stacks of one-dimensional topological superconductors, the other three factors $\mathbb{Z}_{2}$ correspond to stacks of secondorder two-dimensional topological superconductors, the three factors $\mathbb{Z}$ correspond to weak phases consisting of stacks of two-dimensional Chern superconductors, whereas the factor $\mathbb{Z}_{4}$ consists of (strong) second- and third-order topological superconductors, as described above. Following the notation of Ref. [39], Fig 5 lists the appropriate boundary-resolved subgroup sequence $\mathcal{K}_{i}^{\prime \prime} \subset \mathcal{K}_{i}^{\prime} \subset \mathcal{K}$ for each of the factors $\mathcal{K}_{i}$ contained in the full classification group $\mathcal{K}=\prod_{i} \mathcal{K}_{i}$, where $\mathcal{K}_{i}^{\prime}$ and $\mathcal{K}_{i}^{\prime \prime}$ are the subgroups of $\mathcal{K}$ containing topological phases with boundary signature of order larger than one or two, respectively. Weak phases have a shorter subgroup sequence because they do not admit boundary states of order larger than one or two for stacks of one-dimensional topological phases or two-dimensional topological phases, respectively.

Symmetry-based indicators corresponding to the class considered in this example were previously considered in Refs. [65-67]. The explicit expression for $z_{3}$ in Ref. [66] in the weak-pairing limit differs from our Eqs. (110) and (114) by the absence of the sign factors in these references. (Reference [67] contains no explicit expression for the symmetry-based indicators; Ref. [65] defines a $\mathbb{Z}_{2}$ invariant only, for which the sign factors are not important.) This difference affects 
the assignment of a strong (higher-order) topological index $z_{3}$ to weak phases, such as those described by the Hamiltonian $H_{(2, x)}(\mathbf{k})$ of Eq. (111). The assignment of a strong index to these weak phases is ambiguous, as the presence of gapless surfaces in the weak phases makes it impossible to uniquely associate a boundary signature with a nonzero value of the strong indicator in a weak phase. We here follow the convention of Refs. [29] and [80], according to which weak phases are constructed as stack of layers containing the unit-cell center, i.e., with momentum-independent representations.

\section{B. Inversion symmetry, class DIII}

Representation $\Theta=A_{g}$. Bogoliubov-de Gennes-type Hamiltonians in tenfold-way class DIII with inversion symmetry and with a superconducting order parameter transforming according to the $A_{g}$ representation have a trivial classifying group $\mathfrak{K}_{\text {DIII }}\left[C_{i}, A_{g}\right]=\mathfrak{K}_{\text {DIII }}[0]^{2}=0$ at the high-symmetry momenta, so that no topological band labels can be defined. Also, the boundary classifying group of topological phases $\mathcal{K}=0$ is trivial in this symmetry class in three dimensions [39].

Representation $\Theta=A_{u}$. For the $A_{u}$ representation we have the classifying group of inversion-symmetric momenta and Wyckoff positions $\mathfrak{K}_{\mathrm{DIII}}\left[C_{i}, A_{u}\right]=\mathfrak{K}_{\mathrm{AII}}[0]=\mathbb{Z}$. The results for class DIII can be constructed from the class D results by taking the direct sum

$$
H_{\text {DIII }}(\mathbf{k})=H(\mathbf{k}) \oplus_{\sigma} H(-\mathbf{k})^{*},
$$

where $H(\mathbf{k})$ is a class-D Hamiltonian satisfying particle-hole antisymmetry and inversion symmetry with representation $\Theta=A_{u}$. Time- reversal is represented as $\mathcal{T}=\sigma_{2} K$; particlehole conjugation and inversion are diagonal with respect to the $\sigma$ degree of freedom, where it is important that the representation for inversion be real. With the relation (118) the construction of symmetry-based indicators is the same as in class $\mathrm{D}$, with the exception of the $2 \mathrm{D}$ compatibility relation (105), which does not apply to class DIII since no Chern numbers can be defined at two-dimensional planes in the Brillouin zone.

The boundary classifying group for class DIII is

$$
\mathcal{K}_{\text {DIII }}\left[C_{i}, A_{u}\right]=\mathbb{Z}_{2}^{3} \times \mathbb{Z}_{4}^{4} \times \mathbb{Z} \text {. }
$$

With the exception of the one factor $\mathbb{Z}$, the interpretation of these factors and their relation to the topological band labels is the same as for class D. In particular, the construction (118) takes an inversion-symmetric Kitaev chain to its time-reversed counterpart and each Chern superconductor with odd Chern number to a two-dimensional topological superconductor with helical Majorana edge states. Both the even-Chern-number superconductor and the two-dimensional second-order topological superconductor in class D map to the two-dimensional second-order topological superconductor in class DIII. Upon adding weak odd-parity superconducting correlation to an inversion-symmetric quantum spin Hall insulator or threedimensional strong topological insulator in class AII, the edge states gap and create a second-order topological superconductor with a Kramers pair of Majorana corner states or helical Majorana hinge states, respectively. Correspondingly, the inversion-symmetry-protected second-order topological insulator in class AII turns into an odd-parity third-order
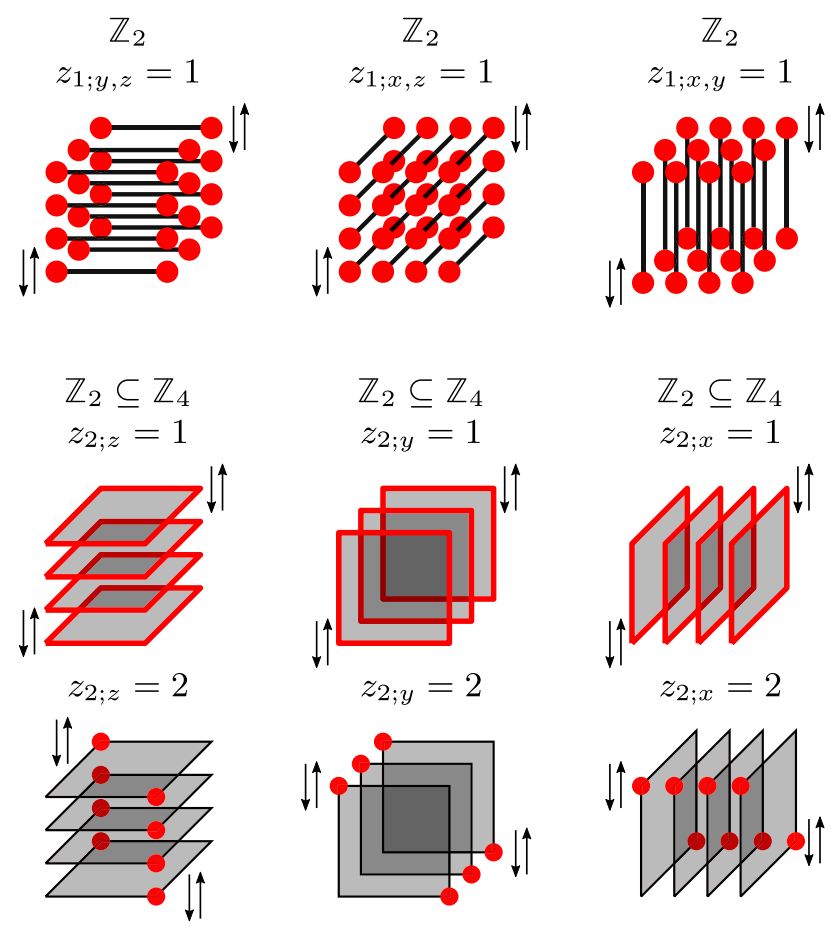

$$
\begin{gathered}
0 \subseteq 0 \subseteq \mathbb{Z} \\
z_{3}=1
\end{gathered}
$$
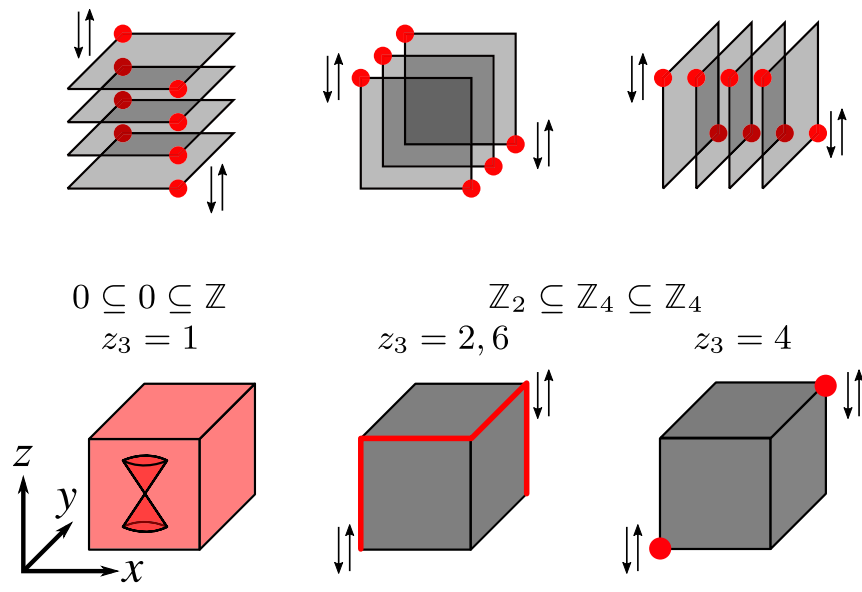

FIG. 6. Topological phases of a three-dimensional superconductor in tenfold-way class DIII, with additional $C_{i}$ symmetry and onedimensional representation $\Theta=A_{u}$. For each boundary signature, the subgroup sequence and the symmetry-based indicators of the generators of that phase are given. The two-dimensional secondorder topological superconductor with $z_{2 ; l}=2$ can be constructed as the direct sum of two two-dimensional first-order topological superconductors with $z_{2 ; l}=1, l=x, y, z$. Similarly, the three-dimensional third-order topological superconductor with $z_{3}=4$ can be constructed as the direct sum of two three-dimensional second-order topological superconductors with $z_{3}=2$.

topological superconductor. For the remaining factor $\mathbb{Z}$, we observe that the construction (118) maps the representationinduced nodal superconductor in class $\mathrm{D}$, which has difference in Chern numbers for parallel planes cutting through the Brillouin zone, to the generator of the three-dimensional (gapped) time-reversal-symmetric topological superconductors, which has indicator $z_{3}=1$. The classification results together with the subgroup sequences are illustrated in Fig. 6.

The generator of the first-order time-reversal-symmetric topological superconductor is

$$
\begin{aligned}
H_{(3)}^{\mathrm{DIII}}(\mathbf{k})= & \sigma_{2} \tau_{2}\left(3-m-\cos k_{x}-\cos k_{y}-\cos k_{z}\right) \\
& +\sigma_{1} \tau_{0} \sin k_{x}+\sigma_{2} \tau_{3} \sin k_{y}+\sigma_{3} \tau_{0} \sin k_{z},
\end{aligned}
$$


with $0<m<2$ where we used the representations

$$
U(\mathcal{P})=\sigma_{0} \tau_{1}, U(\mathcal{I})=\sigma_{2} \tau_{2}, U(\mathcal{T})=\sigma_{2} \tau_{0} .
$$

Direct summation of this phase generates the free $(\mathbb{Z})$ group of first-order topological superconducting phases. Direct summation of the two generators as $H_{(3)}^{\mathrm{DIII}} \otimes \mu_{3}$, with inversion represented as $U(\mathcal{I}) \otimes \mu_{3}$, constructs the generator of the factor $\mathbb{Z}_{4}$ in Eq. (119), a second-order TSC that becomes a third-order topological superconductor upon taking the direct sum with itself. Consequently, all "even" symmetry-based indicators belonging to the factor $\mathbb{Z}_{8}$ are ambiguous: The elements $z_{3}=2$ or 6 and $z_{3}=4$ may correspond to two or four copies of the strong three-dimensional first-order TSC or to the strong second- or third-order topological superconductor, respectively.

The group of symmetry indicators $\mathrm{SI}=\mathbb{Z}_{2}^{3} \times \mathbb{Z}_{4}^{3} \times \mathbb{Z}_{8}$ that we obtain agrees with results from Refs. [67,68]. Reference [65] finds a subgroup $\mathbb{Z}_{2}^{3} \otimes \mathbb{Z}_{4} \subseteq$ SI consisting of the "even" elements only. The explicit expressions for the symmetrybased indicators differ from those in Refs. [65-67] by the presence of the sign factors (see the discussion at the end of Sec. VII A). ${ }^{4}$ The expression for the symmetry-based indicator for strong phases $z_{3}$ in Eq. (114) agrees with the corresponding expression in Ref. [68]. However, in Ref. [68] the symmetry-based indicators for the weak phases are defined on planes or lines with $k_{l}=k_{m}=0$, while our symmetry-based indicators $z_{1 ; l, m}$ and $z_{2 ; l}$ are defined on planes or lines with $k_{l}=k_{m}=\pi$. This definition ensures that no spurious weak indices are assigned to other weak phases or to strong phases. In the weak-pairing limit, the criterion $z_{3}=1 \bmod 2$ for the three-dimensional first-order topological superconductor agrees with the well-known condition that there must be an odd number of Fermi level crossings between high-symmetry momenta (see Refs. [77,78]).

Very recently, the possibility of hybrid higher-order topology was discussed in the literature [81]. We note that for such "hybrid" of first- and second-order topology, it is sufficient to consider inversion-symmetric superconductor as the current example shows: A "hybrid" phase can be identified as a direct sum of a first-order phase (last row, first column in Fig. 6) and one of the weak phases from the third row of Fig. 6.

\section{Inversion symmetry, class C}

General considerations. With spin-rotation symmetry, the Hamiltonian is of the form $H(\mathbf{k})=H_{C}(\mathbf{k}) \otimes \sigma_{0}$ where $H_{C}(\mathbf{k})$ satisfies an effective particle-hole antisymmetry $\mathcal{P}=\tau_{2} K$ squaring to -1 . This is the tenfold-way class $\mathrm{C}$.

Representation $\Theta=A_{g}$. With the $A_{g}$ representation for the transformation behavior of the superconducting order parameter, the classifying group of the Hamiltonian at each of the

\footnotetext{
${ }^{4}$ For symmetry class DIII, the gapless surface states of the weak phases can be removed by breaking translation symmetry. If inversion symmetry is maintained, a strong higher-order phase can result, which has helical Majorana modes along hinges. Such breaking of translation symmetry requires a doubling of the unit cell. Our convention that Hamiltonians such as Eq. (111) have no strong index requires that the inversion center is shifted by half a unit cell upon doubling the unit cell (see, e.g., the discussion in Ref. [80]).
}

high-symmetry momenta is trivial, $\mathfrak{K}_{C}\left[C_{i}, A_{g}\right]=\mathfrak{K}_{C}[0]^{2}=0$, so that no topological band labels can be defined. The classifying group is

$$
\mathcal{K}_{\mathrm{C}}\left[C_{i}, A_{g}\right]=\mathbb{Z}^{3} \times \mathbb{Z}_{2}
$$

(see Ref. [39]). It contains a factor $\mathbb{Z}^{3}$ corresponding to weak Chern superconductors in all three stacking directions and a factor $\mathbb{Z}_{2}$ corresponding to a strong second-order TSC with chiral hinge states. None of those phases can be detected by symmetry-based indicators. The boundary signatures of all topological phases in this class are pairs of chiral Majorana modes. In the limit of vanishing superconducting correlations, they can be adiabatically deformed to corresponding normalstate topological insulators with chiral fermionic modes on their boundaries. Our results for the $\Theta=A_{g}$ representation are consistent with the results of Ref. [67].

Representation $\Theta=A_{u}$. The discussion in class $\mathrm{C}$ with the one-dimensional representation $\Theta=A_{u}$ of inversion symmetry is analogous to the discussion in class D. Each of the high-symmetry momenta and each of the Wyckoff positions come with the classification group $\mathfrak{K}_{\mathrm{C}}\left[C_{i}, A_{u}\right]=\mathfrak{K}_{\mathrm{A}}[0]=\mathbb{Z}$. Compatibility relations of $0 \mathrm{D}$ type follow from the observation that the product $\mathcal{I P}$ acts as an effective particle-hole symmetry that is local in reciprocal space and squares to one. As a result, one may define a Pfaffian invariant $\mathfrak{p}(\mathcal{I} P)$ for $H(\mathbf{k})$ at generic $\mathbf{k}$. Relating $\mathfrak{p}(\mathcal{I} P)$ to the integer invariants $\mathfrak{N}_{+}^{\mathbf{k}_{\mathrm{s}}}$ at the eight high-symmetry momenta $\mathbf{k}_{\mathrm{s}}$, we obtain the seven compatibility relations

$$
\mathfrak{N}_{+}^{\mathbf{k}_{\mathrm{s}}}=\mathfrak{N}_{+}^{(\pi, \pi, \pi)} \bmod 2
$$

for $\mathbf{k}_{\mathrm{s}} \neq(\pi, \pi, \pi)$. Defining the integer band labels

$$
\mathfrak{n}_{+}^{\mathbf{k}_{\mathrm{s}}}=\left(\mathfrak{N}_{+}^{\mathbf{k}_{\mathrm{s}}}-\mathfrak{N}_{+}^{(\pi, \pi, \pi)}\right) / 2,
$$

we arrive at

$$
\begin{aligned}
\mathrm{BS}= & \mathbb{Z}^{8}, \\
B[H(\mathbf{k})]= & \left\{\mathfrak{n}_{+}^{(0,0,0)}, \mathfrak{n}_{+}^{(\pi, 0,0)}, \mathfrak{n}_{+}^{(0, \pi, 0)}, \mathfrak{n}_{+}^{(\pi, \pi, 0)},\right. \\
& \left.\mathfrak{n}_{+}^{(0,0, \pi)}, \mathfrak{n}_{+}^{(\pi, 0, \pi)}, \mathfrak{n}_{+}^{(0, \pi, \pi)}, \mathfrak{N}_{+}^{(\pi, \pi, \pi)}\right\} .
\end{aligned}
$$

The group SI of symmetry-based indicators is

$$
\mathrm{SI}_{\mathrm{C}}\left[C_{i}, A_{u}\right]=\mathbb{Z}_{2}^{3} \times \mathbb{Z}_{4}
$$

An additional compatibility relation of 2D type follows by noting that for a gapped Hamiltonian a Chern number can be defined on planes with fixed $k_{l}$ with $l=x, y$, or $z$ and that this Chern number is always even. At high-symmetry planes with $k_{l}=0$ or $\pi$, the Chern number $\mathrm{Ch}_{l}$ is related to the topological band labels as

$$
\begin{aligned}
\frac{1}{2} \mathrm{Ch}_{l} & =\sum_{\left.\mathbf{k}_{\mathrm{s}}\right|_{k_{\mathrm{s}, l}=0}} \mathfrak{n}_{+}^{\mathbf{k}_{\mathrm{s}}} \bmod 2 \\
& =\sum_{\mathbf{k}_{\mathrm{s}} \neq\left.(\pi, \pi, \pi)\right|_{k_{\mathrm{s}, l}=\pi}} \mathfrak{n}_{+}^{\mathbf{k}_{\mathrm{s}}} \bmod 2 .
\end{aligned}
$$




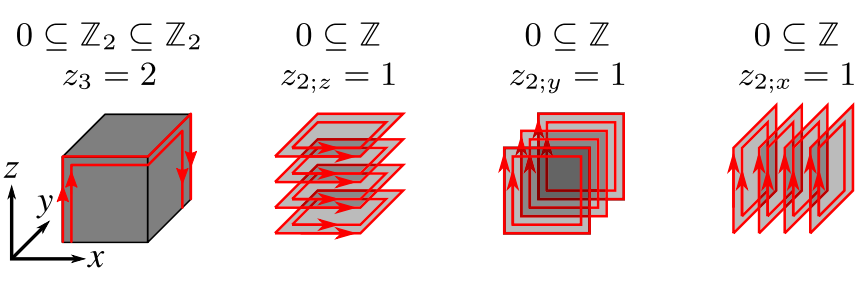

FIG. 7. Topological phases of a three-dimensional superconductor in tenfold-way class $\mathrm{C}$, with additional $C_{i}$ symmetry and onedimensional representation $\Theta=A_{u}$. For each boundary signature, the subgroup sequence and the symmetry-based indicators of the generators of that phase are given.

One verifies that the generator of the factor $\mathbb{Z}_{4}$ in Eq. (124), which has symmetry-based indicator

$$
z_{3}=\sum_{\mathbf{k}_{\mathrm{s}} \neq(\pi, \pi, \pi)} \mathfrak{n}_{+}^{\mathbf{k}_{\mathrm{s}}}(-1)^{\left(k_{\mathrm{s}, x}+k_{\mathrm{s}, y}+k_{\mathrm{s}, z}\right) / \pi} \bmod 4,
$$

is a representation-enforced nodal superconductor, which violates the compatibility relation (125). The three factors $\mathbb{Z}_{2}$ with indicators

$$
z_{2 ; i}=\sum_{\mathbf{k}_{\mathrm{s}} \neq(\pi, \pi, \pi) \mid k_{s, i=\pi}} \mathfrak{n}_{+}^{\mathbf{k}_{\mathrm{s}}} \bmod 2, \quad i=x, y, z
$$

correspond to weak Chern superconductor phases (see Fig. 7).

For comparison, we note that the boundary classification group is [39]

$$
\mathcal{K}_{\mathrm{C}}\left[C_{i}, A_{u}\right]=\mathbb{Z}^{3} \times \mathbb{Z}_{2}
$$

Here, the three factors $\mathbb{Z}$ correspond to weak Chern superconductors with generator Hamiltonians

$$
\begin{aligned}
H_{(2, x)}^{\mathrm{C}}(\mathbf{k})= & \mu_{0} \tau_{3}\left(2-m-\cos k_{y}-\cos k_{z}\right) \\
& +\mu_{1} \tau_{1} \sin k_{y}+\mu_{1} \tau_{2} \sin k_{z}, \text { cycl. }
\end{aligned}
$$

with $0<m<2$. The corresponding representations are

$$
U(\mathcal{P})=\mu_{3} \tau_{2}, \quad U(\mathcal{I})=\mu_{0} \tau_{3} .
$$

The Hamiltonians $H_{(2, l)}^{\mathrm{C}}$ have Chern number $\mathrm{Ch}_{l}=2$ and generate the elements " 1 " in the three factors $\mathbb{Z}_{2}$ of SI [see Eq. (124)]. The remaining factor $\mathbb{Z}_{2}$ of SI is generated by a second-order topological superconductor with chiral hinge states

$$
\begin{aligned}
H_{(3)}^{\prime \mathrm{C}}(\mathbf{k})= & \mu_{0} \rho_{3} \tau_{3}\left(3-m-\cos k_{x}-\cos k_{y}-\cos k_{z}\right) \\
& +\mu_{1} \rho_{0} \tau_{1} \sin k_{x}+\mu_{1} \rho_{0} \tau_{2} \sin k_{y}+\mu_{1} \rho_{1} \tau_{3} \sin k_{z}
\end{aligned}
$$

with $0<m<2$, where we used the representations

$$
U(\mathcal{P})=\mu_{3} \rho_{0} \tau_{2}, \quad U(\mathcal{I})=\mu_{0} \rho_{3} \tau_{3} .
$$

The above phase has symmetry-based indicator $z_{3}=2$ (see Fig. 7). As for the $A_{g}$ representation, the boundary signatures of all topological phases in this class are pairs of chiral Majorana modes. They can be adiabatically deformed to corresponding normal-state topological insulators with chiral fermionic boundary modes.

Our result (124) for the group of symmetry-based indicators is more constrained than the corresponding result from Ref. [67], which finds SI $=\mathbb{Z}_{2}^{3} \times \mathbb{Z}_{4}^{3} \times \mathbb{Z}_{8}$. The origin of the difference is that we include the $0 \mathrm{D}$ compatibility constraint arising from the conservation of the Pfaffian invariant.

\section{Inversion symmetry, class CI}

Representation $\Theta=A_{g}$. Here, the discussion is the same as for class C. Both the group SI of symmetry-based indicators and the classifying group $\mathcal{K}=0$ are trivial [39].

Representation $\Theta=A_{u}$. In the presence of spin-rotation symmetry, time-reversal symmetry can be represented as $\mathcal{T}=$ $K$. Class CI has the same topological band labels as class $\mathrm{C}$, and the generators of atomic limits in classes $\mathrm{C}$ and CI have the same band labels, too. The $0 \mathrm{D}$ compatibility relations (121) continue to be valid. Thus, it follows that the group SI of symmetry-based indicators is again given by Eq. (124).

The higher-dimensional compatibility relations differ from those in class $\mathrm{C}$. On the one hand, there is no Chern number for two-dimensional cuts of the Brillouin zone. On the other hand, $H(\mathbf{k})$ is subject to the chiral antisymmetry $\mathcal{P} T$ and a local-in-k antiunitary symmetry $\mathcal{I} T$ for generic $\mathbf{k}$, which effectively places it in tenfold-way class BDI. For a oneparameter family of gapped Hamiltonians that are (effectively) in class BDI for each $\mathbf{k}$, there exists a $\mathbb{Z}_{2}$ topological invariant, the first Stiefel-Whitney number $[75,76,82]$. Considering this $\mathbb{Z}_{2}$ invariant along one-dimensional cuts through the Brillouin zone and using its relation to the band labels at high-symmetry momenta, one finds four compatibility relations of 1D type (see Appendix C),

$$
\mathfrak{n}_{+}^{\mathbf{k}_{\mathrm{s}}}-\mathfrak{n}_{+}^{\mathbf{k}_{\mathrm{s}}+\pi \mathbf{e}_{l}}=\mathfrak{n}_{+}^{(\pi, \pi, \pi)-\pi \mathbf{e}_{l}} \bmod 2,
$$

for $k_{\mathrm{s}, l}=0, \mathbf{k}_{\mathrm{s}}+\pi \mathbf{e}_{l} \neq(\pi, \pi, \pi)$, where $\mathbf{e}_{l}$ is the unit vector in the $l$ direction, $l=x, y, z$. These $1 \mathrm{D}$ compatibility relations impose the conditions $z_{2 ; l}=0$ and $z_{3}=0 \bmod 2$ for gapped phases. Phases violating these conditions, corresponding to the elements " 1 " in the three factors $\mathbb{Z}_{2}$ and the elements " 1 " and " 3 " in the factor $\mathbb{Z}_{4}$, are representation-enforced nodal-line superconductors. The group of symmetry-based indicators for phases satisfying all compatibility relations is, hence,

$$
\mathrm{SI}_{\mathrm{CI}}^{(2)}\left[C_{i}, A_{u}\right]=\mathbb{Z}_{2} .
$$

For comparison, we note that the boundary classification group is

$$
\mathcal{K}_{\mathrm{CI}}\left[C_{i}, A_{u}\right]=\mathbb{Z} .
$$

It is generated by a strong first-order phase in three dimension whose boundary signature is illustrated in Fig. 8. Its generator Hamiltonian can be written as

$$
\begin{aligned}
H_{(3)}^{\mathrm{CI}}(\mathbf{k})= & \mu_{0} \rho_{0} \tau_{3}\left(3-m-\cos k_{x}-\cos k_{y}-\cos k_{z}\right) \\
& +\mu_{2} \rho_{1} \tau_{1} \sin k_{x}+\mu_{0} \rho_{2} \tau_{1} \sin k_{y}+\mu_{2} \rho_{3} \tau_{1} \sin k_{z}
\end{aligned}
$$

with $0<m<2$. Here, we used the representations

$$
U(\mathcal{P})=\mu_{0} \rho_{0} \tau_{2}, U(\mathcal{I})=\mu_{0} \rho_{0} \tau_{3}, U(\mathcal{T})=\mu_{0} \rho_{0} \tau_{0} .
$$

It has band labels $\mathfrak{n}_{+}^{0}=2, \mathfrak{N}_{+}^{(\pi, \pi, \pi)}=0$, and $\mathfrak{n}_{+}^{\mathbf{k}_{\mathrm{s}}}=0$ for $\mathbf{k}_{\mathrm{s}} \neq$ $(0,0,0),(\pi, \pi, \pi)$. Its band labels generate the element "2" in the factor $\mathbb{Z}_{4}$ of SI and the element " 1 " in the factor $\mathbb{Z}_{2}$ of $\mathrm{SI}^{(2)}$ [see Eq. (131)]. 


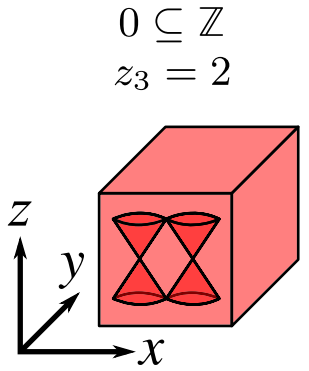

FIG. 8. In tenfold-way class CI with additional $C_{i}$ symmetry and one-dimensional representation $\Theta=A_{u}$, there is a single topological phase with first-order boundary signature consisting of pairs of Majorana Dirac cones. The subgroup sequence and the symmetrybased indicator of the generator of this phase is given.

Similar to the previous section, our group (124) of symmetry-based indicators is more constrained than the corresponding result from Ref. [67], as we include the compatibility relation due to the conservation of the Pfaffian invariant. As discussed above, one finds an even smaller group of symmetry-based indicators if 1D compatibility relations based on the Stiefel-Whitney number are included as well.

\section{CONCLUSION}

Symmetry-based indicators have proven to be a pragmatic substitute for a full classification of topological insulator phases using a complete set of topological invariants $[27,28,56,58,59]$. Their main advantage is that symmetrybased indicators are easier to calculate than other types of topological invariants since they require local information in reciprocal space only. On the other hand, a nonzero value of a symmetry-based indicator is sufficient to establish that a phase has nontrivial topology and may, in addition, contain information of the type of anomalous boundary states [24,60].

In this work, we extend the concept of symmetry-based indicators to Hamiltonians of Bogoliubov-de Gennes (BdG) type, which appear in the mean-field theory superconducting phases. Hamiltonians of $\mathrm{BdG}$ type are antisymmetric with respect to particle-hole conjugation. Additionally, for Hamiltonians of BdG type, a crystalline symmetry class is defined by the presence or absence of time-reversal and spin-rotation symmetry, by the point group $G$, and by a one-dimensional representation $\Theta$ of $G$ that describes how the superconducting order parameter $\Delta$ transforms under the crystalline symmetry [69].

Like the symmetry-based indicators for nonsuperconducting insulating phases $[27,28,58,59]$, the symmetry-based indicators for BdG Hamiltonians can be constructed in a fully algorithmic manner. Input for our construction include "band labels," which are the complete set of "zero-dimensional" topological invariants of the BdG Hamiltonian $H\left(\mathbf{k}_{\mathrm{s}}\right)$ evaluated at high-symmetry points $\mathbf{k}_{\mathrm{s}}$ in the Brillouin zone. Such band labels were first considered by Shiozaki, Sato, and Gomi in Ref. [64]. Unlike previous works on the symmetry-based indicators for topological superconductors [63,65-67], the set of zero-dimensional topological invariants considered by us (and by Ref. [64]) is provably complete: it is not possible to resolve more boundary signatures through the prism of zero-dimensional bulk topological invariants. In particular, the set of topological invariants considered in this work includes not only integer topological invariants of the type used in the construction of symmetry-based indicators for nonsuperconducting Hamiltonians (counting the number of occupied bands corresponding to a certain symmetry representation of the crystalline symmetry), but also $\mathbb{Z}_{2}$ topological invariants constructed using Pfaffians, which do not appear in Refs. [63,65-67]. Pfaffians play a role not only as useful topological band labels, but they also give rise to additional compatibility relations [64], even in cases in which there are symmetry-based indicators of integer type only. The latter point is well illustrated by the example of tenfold-way classes $\mathrm{C}$ and CI with inversion symmetry and $\Theta=A_{u}$, discussed in Secs. VII C and VII D.

In the weak-pairing limit (superconducting gap $\Delta$ small in comparison to characteristic energy scales of the normal-state band structure), the band labels of the BdG Hamiltonian can be expressed in terms of conventional integer band labels of the normal-state Hamiltonian, provided the symmetry of the superconducting order parameter is known $[67,68]$. This applies both to the integer invariant and to the $\mathbb{Z}_{2}$ Pfaffian invariant. This means that in the weak pairing, the symmetrybased indicators constructed here can be calculated using the vast amount of known band-structure data in the normal state.

The main difference between zero-dimensional band labels and symmetry-based indicators [27] is that the latter are designed to "see" only the topological phases with nontrivial boundary signatures. Topologically nontrivial superconductors without gapless boundary states are deformable to atomic-limit phases. In this work, we combine the complete set of zero-dimensional band labels [64] and the definition of atomic-limit superconductors as an "array" of zero-dimensional superconductors [39] to arrive at an extension of symmetry-based indicators [27] to Hamiltonians of Bogoliubov-de Gennes type. Our definition of atomic-limit superconductors agrees with the definitions of Refs. [67,68]. Since Pfaffian invariants do not appear in Refs. [65-67], the symmetry-based indicators we obtain may be expected to be consistent with these references once all topological band labels and all compatibility relations associated with Pfaffian invariants are omitted from our construction. (For inversion-symmetric superconductors, however, our concrete expressions for the symmetry-based indicators differ from those of Refs. [65-67]. This is discussed in detail in Sec. VII.) Reference [68] contains results closely related to and consistent with ours.

If the symmetry-based indicators are used as a substitute for a full classification, ideally, one wants symmetry-based indicators to detect gapped phases only. The algorithmic construction defined in this work partially meets this goal: It only guarantees that no indicator corresponds to gapless topological superconductor phases with its gapless points on high-symmetry lines in Brillouin zone that connect the high-symmetry points. Nevertheless, for the examples considered in this work, we were able to explicitly relate all gapless phases, including gapless phases with gapless points occurring on high-symmetry planes and in the bulk of the 
TABLE XII. The group of symmetry-based indicators SI obtained by including compatibility relations of OD type only, the group $\mathrm{SI}^{(d-1)}$ obtained by including compatibility relations of $n$ dimensional type, with $n<d$, and the full boundary classification group $\mathcal{K}$ for the combinations of tenfold-way classes, dimension $d$, and point group $G$ considered in this work.

\begin{tabular}{|c|c|c|c|c|c|c|}
\hline$d$ & $G$ & Cartan & $\Theta$ & SI & $\mathrm{SI}^{(d-1)}$ & $\mathcal{K}$ \\
\hline \multirow[t]{4}{*}{1} & \multirow[t]{4}{*}{$C_{1}$} & $\mathrm{D}$ & $A$ & $\mathbb{Z}_{2}$ & $\mathbb{Z}_{2}$ & $\mathbb{Z}_{2}$ \\
\hline & & DIII & $A$ & 0 & 0 & $\mathbb{Z}_{2}$ \\
\hline & & $\mathrm{C}$ & $A$ & 0 & 0 & 0 \\
\hline & & $\mathrm{CI}$ & $A$ & 0 & 0 & 0 \\
\hline \multirow[t]{2}{*}{1} & $C_{i}$ & $\mathrm{D}$ & $A_{g}$ & 0 & 0 & 0 \\
\hline & & & $A_{u}$ & $\mathbb{Z}_{2}$ & $\mathbb{Z}_{2}$ & $\mathbb{Z}_{2}$ \\
\hline \multirow[t]{4}{*}{2} & $C_{1}$ & $\mathrm{D}$ & $A$ & $\mathbb{Z}_{2}^{3}$ & $\mathbb{Z}_{2}^{3}$ & $\mathbb{Z}_{2}^{2} \times \mathbb{Z}$ \\
\hline & & DIII & $A$ & 0 & 0 & $\mathbb{Z}_{2}^{3}$ \\
\hline & & $\mathrm{C}$ & $A$ & 0 & 0 & $\mathbb{Z}$ \\
\hline & & CI & $A$ & 0 & 0 & 0 \\
\hline 2 & $C_{i}$ & $\mathrm{D}$ & $\begin{array}{l}A_{g} \\
A_{u}\end{array}$ & $\begin{array}{c}\mathbb{Z}_{2} \\
\mathbb{Z}_{2}^{2} \times \mathbb{Z}_{4}\end{array}$ & $\begin{array}{c}\mathbb{Z}_{2} \\
\mathbb{Z}_{2}^{2} \times \mathbb{Z}_{4}\end{array}$ & $\mathbb{Z}_{2}^{3} \times \mathbb{Z}$ \\
\hline \multirow[t]{2}{*}{2} & $C_{s}$ & $\mathrm{D}$ & $A^{\prime}$ & $\mathbb{Z}_{2}$ & $\mathbb{Z}_{2}$ & $\mathbb{Z}_{2}$ \\
\hline & & & $A^{\prime \prime}$ & $\mathbb{Z}_{2}^{3}$ & $\mathbb{Z}_{2}^{3}$ & $\mathbb{Z}_{2}^{3}$ \\
\hline \multirow[t]{2}{*}{2} & $C_{2}$ & $\mathrm{D}$ & $A$ & $\mathbb{Z}_{2}^{2} \times \mathbb{Z}_{4}$ & $\mathbb{Z}_{2}^{2} \times \mathbb{Z}_{4}$ & $\mathbb{Z}_{2}^{3} \times \mathbb{Z}$ \\
\hline & & & $B$ & $\mathbb{Z}_{2}$ & $\mathbb{Z}_{2}$ & $\mathbb{Z}$ \\
\hline \multirow[t]{4}{*}{2} & $C_{2 v}$ & $\mathrm{D}$ & $A_{1}$ & 0 & 0 & 0 \\
\hline & & & $A_{2}$ & $\mathbb{Z}_{2}^{3}$ & $\mathbb{Z}_{2}^{3}$ & $\mathbb{Z}_{2}^{4}$ \\
\hline & & & $B_{1}$ & $\mathbb{Z}_{2}$ & $\mathbb{Z}_{2}$ & $\mathbb{Z}_{2}$ \\
\hline & & & $B_{2}$ & $\mathbb{Z}_{2}$ & $\mathbb{Z}_{2}$ & $\mathbb{Z}_{2}$ \\
\hline \multirow[t]{2}{*}{2} & $C_{4}$ & $\mathrm{D}$ & $A, B$ & $\mathbb{Z}_{2} \times \mathbb{Z}_{8}$ & $\mathbb{Z}_{2} \times \mathbb{Z}_{8}$ & $\mathbb{Z}_{2}^{2} \times \mathbb{Z}$ \\
\hline & & & ${ }^{1,2} E$ & $\mathbb{Z}_{4}$ & $\mathbb{Z}_{4}$ & $\mathbb{Z}$ \\
\hline \multirow[t]{4}{*}{3} & $C_{1}$ & $\mathrm{D}$ & $A$ & $\mathbb{Z}_{2}^{7}$ & $\mathbb{Z}_{2}^{6}$ & $\mathbb{Z}_{2}^{3} \times \mathbb{Z}^{3}$ \\
\hline & & DIII & $A$ & 0 & 0 & $\mathbb{Z}_{2}^{6} \times \mathbb{Z}$ \\
\hline & & $\mathrm{C}$ & $A$ & 0 & 0 & $\mathbb{Z}^{3}$ \\
\hline & & CI & $A$ & 0 & 0 & $\mathbb{Z}$ \\
\hline \multirow[t]{8}{*}{3} & $C_{i}$ & $\mathrm{D}$ & $A_{g}$ & $\mathbb{Z}_{2}^{4}$ & $\mathbb{Z}_{2}^{3}$ & $\mathbb{Z}^{3}$ \\
\hline & & & $A_{u}$ & $\mathbb{Z}_{2}^{3} \times \mathbb{Z}_{4}^{3} \times \mathbb{Z}_{8}$ & $\mathbb{Z}_{2}^{3} \times \mathbb{Z}_{4}^{4}$ & $\mathbb{Z}_{2}^{6} \times \mathbb{Z}_{4} \times \mathbb{Z}^{3}$ \\
\hline & & DIII & $A_{g}$ & 0 & 0 & 0 \\
\hline & & & $\dot{A_{u}}$ & $\mathbb{Z}_{2}^{3} \times \mathbb{Z}_{4}^{3} \times \mathbb{Z}_{8}$ & $\mathbb{Z}_{2}^{3} \times \mathbb{Z}_{4}^{3} \times \mathbb{Z}_{8}$ & $\mathbb{Z}_{2}^{3} \times \mathbb{Z}_{4}^{4} \times \mathbb{Z}$ \\
\hline & & $\mathrm{C}$ & $A_{g}$ & 0 & 0 & $\mathbb{Z}^{3} \times \mathbb{Z}_{2}$ \\
\hline & & & $A_{u}$ & $\mathbb{Z}_{2}^{3} \times \mathbb{Z}_{4}$ & $\mathbb{Z}_{2}^{4}$ & $\mathbb{Z}^{3} \times \mathbb{Z}_{2}$ \\
\hline & & CI & $A_{g}$ & 0 & 0 & 0 \\
\hline & & & $A_{u}$ & $\mathbb{Z}_{2}^{3} \times \mathbb{Z}_{4}$ & $\mathbb{Z}_{2}$ & $\mathbb{Z}$ \\
\hline \multirow[t]{2}{*}{3} & $C_{s}$ & $\mathrm{D}$ & $A^{\prime}$ & $\mathbb{Z}_{2}$ & $\mathbb{Z}_{2}$ & $\mathbb{Z}_{2} \times \mathbb{Z}$ \\
\hline & & & $A^{\prime \prime}$ & $\mathbb{Z}_{2}^{9}$ & $\mathbb{Z}_{2}^{9}$ & $\mathbb{Z}_{2}^{7} \times \mathbb{Z}^{2}$ \\
\hline \multirow[t]{2}{*}{3} & $C_{2}$ & $\mathrm{D}$ & $A$ & $\mathbb{Z}_{2}^{2} \times \mathbb{Z}_{4}$ & $\mathbb{Z}_{2}^{2} \times \mathbb{Z}_{4}$ & $\mathbb{Z}_{2}^{3} \times \mathbb{Z}$ \\
\hline & & & $B$ & $\mathbb{Z}_{2}^{6}$ & $\mathbb{Z}_{2}^{5}$ & $\mathbb{Z}_{2}^{4} \times \mathbb{Z}$ \\
\hline \multirow[t]{4}{*}{3} & $C_{2 v}$ & $\mathrm{D}$ & $A_{1}$ & 0 & 0 & 0 \\
\hline & & & $A_{2}$ & $\mathbb{Z}_{2}^{7}$ & $\mathbb{Z}_{2}^{7}$ & $\mathbb{Z}_{2}^{5} \times \mathbb{Z}^{4}$ \\
\hline & & & $B_{1}$ & $\mathbb{Z}_{2}^{3}$ & $\mathbb{Z}_{2}^{3}$ & $\mathbb{Z}_{2}^{3} \times \mathbb{Z}^{2}$ \\
\hline & & & $B_{2}$ & $\mathbb{Z}_{2}^{\frac{2}{3}}$ & $\mathbb{Z}_{2}^{3}$ & $\mathbb{Z}_{2}^{3} \times \mathbb{Z}^{2}$ \\
\hline \multirow[t]{2}{*}{3} & $C_{4}$ & $\mathrm{D}$ & $A, B$ & $\mathbb{Z}_{2} \times \mathbb{Z}_{8}$ & $\mathbb{Z}_{2} \times \mathbb{Z}_{8}$ & $\mathbb{Z}_{2}^{2} \times \mathbb{Z}$ \\
\hline & & & ${ }^{1,2} E$ & $\mathbb{Z}_{2}^{4} \times \mathbb{Z}_{4}$ & $\mathbb{Z}_{2}^{3} \times \mathbb{Z}_{4}$ & $\mathbb{Z}_{2}^{3} \times \mathbb{Z}$ \\
\hline
\end{tabular}

Brillouin zone, to symmetry-based indicators by invoking additional compatibility relations that involve winding numbers, Chern numbers, as well as first and second StiefelWhitney numbers. These compatibility relations are defined on one-dimensional and two-dimensional cuts through the Brillouin zone, respectively, involve local-in-k properties of $H(\mathbf{k})$ only, and generalize the compatibility relations that make use of the continuity of zero-dimensional invariants in the Brillouin zone [27,28,56]. Formally, the inclusion of higher- (up to $n$-) dimensional compatibility relations allows one to define a smaller group $\mathrm{SI}^{(n)} \subset \mathrm{SI}$ of symmetry-based indicators. The relevant (smallest) groups $\mathrm{SI}^{(d-1)}$ are listed in Table XII for the examples considered. For the examples we considered, we find that all phases indexed by $\mathrm{SI}^{(d-1)}$ are gapped. It would be interesting to find out, whether this feature holds in general, i.e., whether the inclusion of these two "higher-dimensional" compatibility relations involving local-in-k symmetries of $H(\mathbf{k})$ only is sufficient to identify all symmetry-based indicators that correspond to enforced gapless phases.

To see to what extent symmetry-based indicators offer a faithful representation of all (crystalline) topological phases, we compared the symmetry-based indicators with the complete classification information for selected examples. To this end, we used the classifying group $\mathcal{K}$, which classifies all topological phases with protected boundary states. (This excludes atomic-limit phases with nontrivial topology from the topological classification, which is consistent with the fact that symmetry-based indicators of atomic-limit phases are defined to be zero [27,28].) A summary of this comparison is shown in Table XII. Only for a small number of the examples we consider, such as tenfold-way class $\mathrm{D}$ in three dimensions with symmetry groups $C_{s}$ or $C_{2 v}$ and representations $\Theta=A^{\prime}$ and $B_{2}$, respectively, entire classes of topological phases are missed by the symmetry-based indicators, whereas for some crystalline symmetry classes the full classifying group $\mathcal{K}$ and the group SI of symmetry-based indicators are identical. In most cases, all generators of topological phases are detectable by symmetry-based indicators, although there may be ambiguities preventing a unique identification of the precise nature of the topological phase. Although these examples clearly show that symmetry-based indicators are not equivalent to a complete classification, it remains an interesting observation that zero-dimensional invariants alone perform so well at this task.

Note added. Recently, Refs. [67,68] appeared.

\section{ACKNOWLEDGMENTS}

We thank A. Bernevig, T. Neupert, S. Ono, F. von Oppen, K. Shiozaki, and H. Watanabe for stimulating discussions. We acknowledge support by project A03 of the CRC-TR 183 (M.G. and P.W.B.) and from the FNS/SNF Ambizione Grant No. PZ00P2_179962 (L.T.).

\section{APPENDIX A: MORE EXAMPLES IN THREE DIMENSIONS}

We discuss the classifying group $\mathcal{K}_{\eta}[G, \Theta]$, band labels, compatibility relations, and symmetry-based indicators of the 
topological phases with nontrivial boundary signatures for a selection of point groups not covered in the main text.

\section{Trivial point group $C_{1}$, classes $\mathrm{D}, \mathrm{DIII}, \mathrm{C}, \mathrm{CI}$}

In the absence of crystalline symmetries (other than translation), for classes DIII, C, and CI there are no topological band labels, as the classification of the inversion-symmetric momenta $\mathfrak{K}_{\eta}\left[C_{1}, A\right]$ is trivial for those classes. The classifying groups are $\mathcal{K}_{\mathrm{DIII}}\left[C_{1}, A\right]=\mathbb{Z}_{2}^{6} \times \mathbb{Z}$, with three factors $\mathbb{Z}_{2}$ for weak phases corresponding to stacks of onedimensional time-reversal-symmetric topological superconductors, three factors $\mathbb{Z}_{2}$ for stacks of two-dimensional timereversal-symmetric topological superconductors, and one factor $\mathbb{Z}$ for a three-dimensional strong first-order superconductor phase $\mathcal{K}_{\mathrm{C}}\left[C_{1}, A\right]=\mathbb{Z}^{3}$, the three factors $\mathbb{Z}$ describing weak phases corresponding to stacks of two-dimensional Chern superconductors with even Chern number, which can be adiabatically deformed to normal-state Chern insulators, and $\mathcal{K}_{\mathrm{CI}}\left[C_{1}, A\right]=\mathbb{Z}$, corresponding to a three-dimensional strong phase. None of these phases can be detected using symmetry-based indicators. The symmetry-based indicators for tenfold-way class D are nontrivial, as we discuss below.

Classifying group. The boundary classifying group for tenfold-way class $\mathrm{D}$ is

$$
\mathcal{K}_{\mathrm{D}}\left[C_{1}, A\right]=\mathbb{Z}_{2}^{3} \times \mathbb{Z}^{3} .
$$

The factor $\mathbb{Z}_{2}^{3}$ corresponds to Kitaev chains stacked in the $y$ and $z, x$ and $z$, or $x$ and $y$ directions [labels $(1 ; y, z),(1 ; x, z)$, and $(1 ; x, y)]$ and the factor $\mathbb{Z}^{3}$ corresponds to Chern superconductors stacked in the $z, y$, or $x$ direction [labels $(2 ; z)$, $(2 ; y)$, and $(2 ; x)]$. The even-Chern-number superconductors can be deformed to Chern insulators with vanishing superconducting order parameter. The topological phases generate a $\mathbb{Z}_{2}^{6}$ subgroup of $\mathrm{SI}_{\mathrm{D}}\left[C_{1}, A\right]=\mathbb{Z}_{2}^{7}$. The boundary signatures of the topological phases together with their symmetry-based indicators are shown in Fig. 9.

Band labels. There are $\mathrm{BL} \simeq \mathbb{Z}_{2}^{8}$ topological band labels given by the Pfaffian invariant $\mathfrak{p}^{\mathbf{k}_{\mathbf{s}}}$ at all high-symmetry momenta $\mathbf{k}_{\mathrm{s}}$.

Compatibility relations. There are no OD compatibility relations. Hence, $\mathrm{BL} \simeq \mathrm{BS}$ and

$$
B[H(\mathbf{k})]=\left\{\mathfrak{p}^{\mathbf{k}_{\mathrm{s}}}\right\} .
$$

There is a $2 \mathrm{D}$ compability relation required by the conservation of the Chern number between parallel planes,

$$
\sum_{\mathbf{k}_{\mathrm{s}} \mid k_{\mathrm{s}, i}=0} \mathfrak{p}^{\mathbf{k}_{\mathrm{s}}}=\sum_{\mathbf{k}_{\mathrm{s}} \mid k_{\mathrm{s}, i}=\pi} \mathfrak{p}^{\mathbf{k}_{\mathrm{s}}} \bmod 2, \quad i=x, y, z
$$

The violation of this compatibility relation signals a gapless phase with nodal points.

Symmetry-based indicators. The group of symmetry-based indicators

$$
\mathrm{SI}_{\mathrm{D}}\left[C_{1}, A\right]=\mathrm{BS} / \mathrm{AI} \simeq \mathbb{Z}_{2}^{7}
$$

contains a factor $\mathbb{Z}_{2}^{6}$ corresponding to stacks of one- and twodimensional topological superconductors,

$$
z_{1 ; i, j}=\sum_{\mathbf{k}_{\mathrm{s}} \mid k_{s, i}=k_{\mathrm{s}, j}=\pi} \mathfrak{p}^{\mathbf{k}_{\mathrm{s}}} \bmod 2,
$$

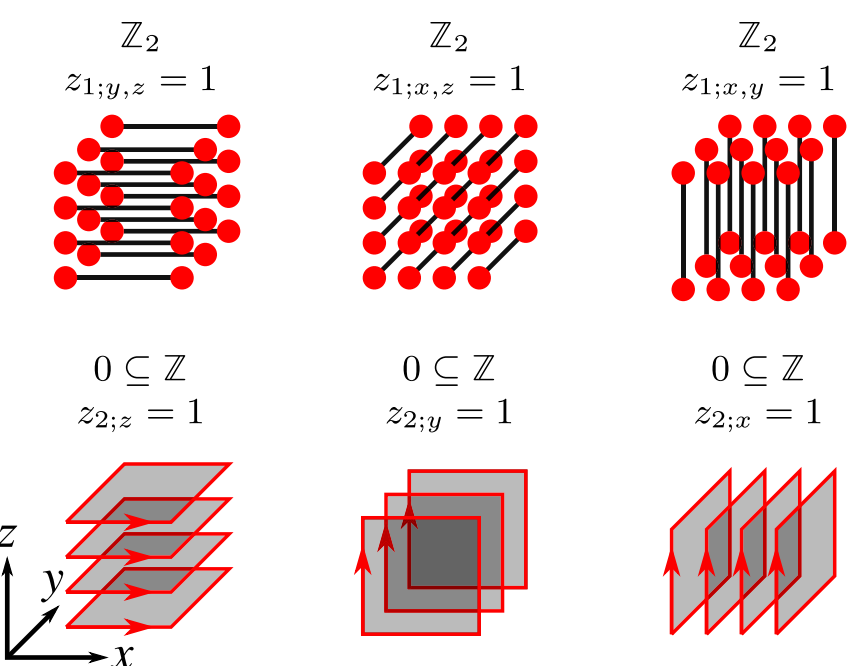

FIG. 9. Topological phases of a three-dimensional superconductor in tenfold-way class D and with translation symmetry only (point group $C_{1}$ ). For each boundary signature, the boundary subgroup sequence (top row) and the nonzero symmetry-based indicators for a generator of that phase are given (middle row).

$$
z_{2 ; j}=\sum_{\mathbf{k}_{\mathrm{s}} \mid k_{\mathrm{s}, j}=\pi} \mathfrak{p}^{\mathbf{k}_{\mathrm{s}}} \bmod 2,
$$

with $i \neq j=x, y, z$. The remaining factor $\mathbb{Z}_{2}$ corresponds to a nodal superconductor detected by the violation of the $2 \mathrm{D}$ compatibility relation with symmetry-based indicator

$$
z_{3}=\sum_{\mathbf{k}_{\mathrm{s}}} \mathfrak{p}^{\mathbf{k}_{\mathrm{s}}} \bmod 2 .
$$

\section{Point group $C_{s}$, class $\mathrm{D}$ \\ a. Representation $\Theta=A^{\prime}$}

Classifying group:

$$
\mathcal{K}_{\mathrm{D}}\left[C_{s}, A^{\prime}\right]=\mathbb{Z}_{2} \times \mathbb{Z} .
$$

The factor $\mathbb{Z}_{2}$ corresponds to a stack of mirror-symmetric Kitaev chains in the $y$ and $z$ directions [label $(1 ; y, z)]$ and the factor $\mathbb{Z}$ corresponds to a stack of two-dimensional Chern superconductors with even Chern number within the mirror plane [label $(2 ; x)]$. The latter phase can not be detected by symmetry-based indicators. It can be adiabatically deformed to a Chern insulator that is robust to the introduction of oddparity superconducting correlations. The boundary signatures of the topological phases together with their symmetry-based indicators are displayed in Fig. 10.

Band labels. There are $\mathrm{BL}=\mathbb{Z}^{8}$ topological band labels given by the invariants $\mathfrak{N}_{+}^{\mathbf{k}_{\mathbf{s}}}$ at all high-symmetry momenta $\mathbf{k}_{\mathrm{s}}$.

Compatibility relations. Within a mirror plane $k_{x}=0, \pi$ the invariant $\mathfrak{N}_{+}^{\mathbf{k}}$ is preserved for a gapped phase, which leads to the $0 \mathrm{D}$ compatibility relations

$$
\begin{aligned}
\mathfrak{N}_{+}^{(0,0,0)} & =\mathfrak{N}_{+}^{(0, \pi, 0)}=\mathfrak{N}_{+}^{(0,0, \pi)}=\mathfrak{N}_{+}^{(0, \pi, \pi)}, \\
\mathfrak{N}_{+}^{(\pi, 0,0)} & =\mathfrak{N}_{+}^{(\pi, \pi, 0)}=\mathfrak{N}_{+}^{(\pi, 0, \pi)}=\mathfrak{N}_{+}^{(\pi, \pi, \pi)},
\end{aligned}
$$

which identifies a factor $\mathbb{Z}^{6}$ of the group of topological band labels BL as representation-enforced gapless nodal-line 

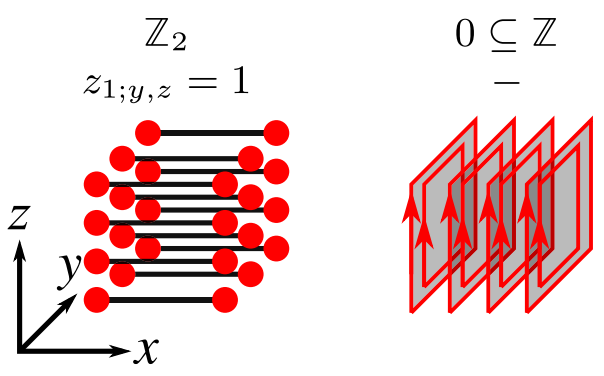

FIG. 10. Topological phases of a three-dimensional superconductor in tenfold-way class $\mathrm{D}$ with point group $C_{s}$ and representation $\Theta=A^{\prime}$.

superconductors such that we have $\mathrm{BS} \simeq \mathbb{Z}^{2}$ and

$$
B[H(\mathbf{k})]=\left\{\mathfrak{N}_{+}^{(0, \pi, \pi)}, \mathfrak{N}_{+}^{(\pi, \pi, \pi)}\right\} .
$$

Symmetry-based indicators. The group of symmetry-based indicators

$$
\mathrm{SI}_{\mathrm{D}}\left[C_{s}, A^{\prime}\right] \simeq \mathbb{Z}_{2}
$$

contains a single factor corresponding to the stack of Kitaev chains with indicator

$$
z_{1 ; y, z}=\mathfrak{N}_{+}^{(\pi, \pi, \pi)}+\mathfrak{N}_{+}^{(0, \pi, \pi)} \bmod 2 .
$$

Our result (A1) for the group of symmetry-based indicators agrees with the corresponding result from [67].

\section{b. Representation $\Theta=A^{\prime \prime}$}

Classifying group:

$$
\mathcal{K}_{\mathrm{D}}\left[C_{s}, A^{\prime \prime}\right]=\mathbb{Z}_{2}^{7} \times \mathbb{Z}^{2} .
$$

A factor $\mathbb{Z}_{2}^{4}$ corresponds to one-dimensional superconductors in the $y$ or $z$ directions, stacked in the $x$ and $z$ or $x$ and $y$ directions, respectively, and with $\mathcal{M}_{x}$ parities \pm [labels $(1, \pm ; x, z)$ and $(1, \pm ; x, y)]$. The factor $\mathbb{Z}^{2}$ corresponds to two-dimensional Chern superconductors in the $y z$ plane with even or odd $\mathcal{M}_{x}$ parity, stacked in the $x$ direction [labels $(2, \pm ; x)]$. Systems with an even Chern number in either mirror plane can be adiabatically deformed to Chern insulator with vanishing superconducting order parameter. A factor $\mathbb{Z}_{2}^{2}$ corresponds to two-dimensional second-order topological superconductors stacked in the $z$ or $y$ direction [labels $(2 ; z)$ and $(2 ; y)]$. The remaining factor $\mathbb{Z}_{2}$ corresponds to a three-dimensional second-order topological superconductor [label (3)]. The boundary signatures of the topological phases together with their symmetry-based indicators are displayed in Fig. 11.

Band labels. There are $\mathrm{BL} \simeq \mathbb{Z}_{2}^{16}$ topological band labels given by the Pfaffian invariants $\mathfrak{p}_{ \pm}^{\mathbf{k}_{\mathrm{s}}}$ in both even and odd mirror parity subspaces at all high-symmetry momenta $\mathbf{k}_{\mathrm{s}}$.

Compatibility relations. From the fact that $\mathcal{P M}$ is a local antisymmetry along lines in reciprocal space, one deduces the compatibility relations

$$
\sum_{s= \pm} \mathfrak{p}_{s}^{\left(0, k_{y}^{\prime}, k_{z}^{\prime}\right)}=\sum_{s= \pm} \mathfrak{p}_{s}^{\left(\pi, k_{y}^{\prime}, k_{z}^{\prime}\right)} \text { for } k_{y}^{\prime}, k_{z}^{\prime}=0, \pi
$$

The compatibility relations identify a factor $\mathbb{Z}_{2}^{4}$ of $\mathrm{BL}$ as representation-enforced gapless superconductors with nodal

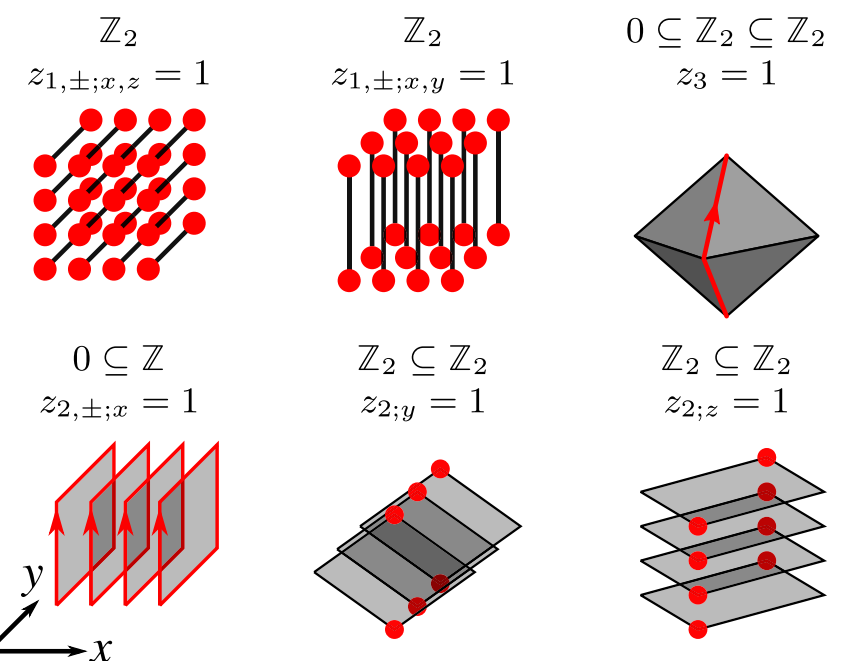

FIG. 11. Topological phases of a three-dimensional superconductor in tenfold-way class $\mathrm{D}$ with point group $C_{s}$ and representation $\Theta=A^{\prime \prime}$.

points on high-symmetry lines in the Brillouin zone such that we have $\mathrm{BS} \simeq \mathbb{Z}_{2}^{12}$ and

$$
\begin{aligned}
B[H(\mathbf{k})]= & \left\{\mathfrak{p}_{-}^{(0,0,0)}, \mathfrak{p}_{-}^{(0,0, \pi)}, \mathfrak{p}_{-}^{(0, \pi, 0)}, \mathfrak{p}_{-}^{(0, \pi, \pi)},\right. \\
& \mathfrak{p}_{+}^{(\pi, 0,0)}, \mathfrak{p}_{+}^{(\pi, 0, \pi)}, \mathfrak{p}_{+}^{(\pi, \pi, 0)}, \mathfrak{p}_{+}^{(\pi, \pi, \pi)}, \\
& \left.\mathfrak{p}_{-}^{(\pi, 0,0)}, \mathfrak{p}_{-}^{(\pi, 0, \pi)}, \mathfrak{p}_{-}^{(\pi, \pi, 0)}, \mathfrak{p}_{-}^{(\pi, \pi, \pi)}\right\} .
\end{aligned}
$$

Symmetry-based indicators. The group of symmetry-based indicators is

$$
\mathrm{SI}_{\mathrm{D}}\left[C_{s}, A^{\prime \prime}\right] \simeq \mathbb{Z}_{2}^{9},
$$

where each factor corresponds to a different topological phase. For the stacks of Kitaev chains we have

$$
\begin{aligned}
& z_{1, \pm ; x, z}=\mathfrak{p}_{ \pm}^{(\pi, \pi, \pi)}+\mathfrak{p}_{ \pm}^{(\pi, 0, \pi)} \bmod 2, \\
& z_{1, \pm ; x, y}=\mathfrak{p}_{ \pm}^{(\pi, \pi, \pi)}+\mathfrak{p}_{ \pm}^{(\pi, \pi, 0)} \bmod 2,
\end{aligned}
$$

for the Chern superconductors within the mirror plane with even or odd mirror parity

$$
z_{2, \pm ; x}=\sum_{\left.\mathbf{k}_{s}\right|_{k_{s, x}=\pi}} \mathfrak{p}_{ \pm}^{\mathbf{k}_{\mathrm{s}}} \bmod 2,
$$

for two-dimensional second-order topological superconductors stacked in the $l=z$ or $y$ direction

$$
z_{2 ; l}=\sum_{\left.\mathbf{k}_{s}\right|_{k_{s, l}=\pi}} \mathfrak{p}_{+}^{\mathbf{k}_{\mathrm{s}}}=\sum_{\mathbf{k}_{\mathrm{s}} \mid k_{\mathrm{s}, l}=\pi} \mathfrak{p}_{-}^{\mathbf{k}_{\mathrm{s}}} \bmod 2,
$$

where the equality follows from the compatibility constraint, and for the three-dimensional second-order topological superconductor we have

$$
z_{3}=\sum_{\mathbf{k}_{\mathrm{s}}} \mathfrak{p}_{+}^{\mathbf{k}_{\mathrm{s}}}=\sum_{\mathbf{k}_{\mathrm{s}}} \mathfrak{p}_{-}^{\mathbf{k}_{\mathrm{s}}} \bmod 2 .
$$

The group (A2) of symmetry-based indicators differs from the result obtained in Ref. [67], where no symmetry-based indicators are found due to the absence of Pfaffian band labels. 

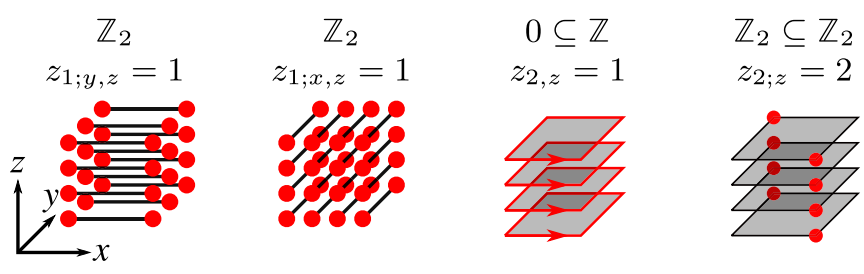

FIG. 12. Topological phases of a three-dimensional superconductor in tenfold-way class $\mathrm{D}$ with point group $C_{2}$ and representation $\Theta=A$.

\section{Point group $C_{2}$, class $\mathrm{D}$ \\ a. Representation $\Theta=A$}

Classifying group:

$$
\mathcal{K}_{\mathrm{D}}\left[C_{2}, A\right]=\mathbb{Z}_{2}^{3} \times \mathbb{Z}
$$

A factor $\mathbb{Z}_{2}^{2}$ corresponds to Kitaev chains perpendicular to the rotation axis $(z)$ and stacked in the $x$ and $z$ or $y$ and $z$ directions [labels $(1 ; y, z)$ and $(1 ; x, z)]$. The remaining factors $\mathbb{Z}_{2}$ and $\mathbb{Z}$ correspond to two-dimensional topological superconductors stacked perpendicular to the rotation axis [labels $(2, z)^{\prime}$ and $(2, z)$, respectively], where the factor $\mathbb{Z}_{2}$ describes a twodimensional second-order phase and the factor $\mathbb{Z}$ a Chern superconductor. Even-Chern-number superconductors can be adiabatically deformed to normal-state Chern insulators. The weak second-order phase and the stack of Chern superconductors with Chern number two have identical band labels. The boundary signatures of the topological phases together with their symmetry-based indicators are displayed in Fig. 12.

Band labels. There are $\mathrm{BL} \simeq \mathbb{Z}^{8}$ topological band labels given by the invariants $\mathfrak{N}_{+}^{\mathbf{k}_{\mathrm{s}}}$ at all high-symmetry momenta $\mathbf{k}_{\mathrm{s}}$.

Compatibility relations. For gapped phases, the invariant $\mathfrak{N}_{+}(\mathbf{k})$ is preserved along high-symmetry lines in reciprocal space parallel to the rotation axis, which gives the compatibility relation

$$
\mathfrak{N}_{+}^{\left(k_{x}^{\prime}, k_{y}^{\prime}, 0\right)}=\mathfrak{N}_{+}^{\left(k_{x}^{\prime}, k_{y}^{\prime}, \pi\right)} \text { for } k_{x}^{\prime}, k_{y}^{\prime}=0, \pi
$$

This compatibility relation identifies a factor $\mathbb{Z}^{4}$ of BL as representation-enforced gapless superconductors with nodal points such that we have $\mathrm{BS} \simeq \mathbb{Z}^{4}$ and

$$
B[H(\mathbf{k})]=\left\{\mathfrak{N}_{+}^{(0,0, \pi)}, \mathfrak{N}_{+}^{(\pi, 0, \pi)}, \mathfrak{N}_{+}^{(0, \pi, \pi)}, \mathfrak{N}_{+}^{(\pi, \pi, \pi)}\right\} .
$$

Symmetry-based indicators. The group of symmetry-based indicators is

$$
\mathrm{SI}_{\mathrm{D}}\left[C_{2}, A\right] \simeq \mathbb{Z}_{2}^{2} \times \mathbb{Z}_{4}
$$

The factor $\mathbb{Z}_{2}^{2}$ correspond to stacks of Kitaev chains with indicators

$$
\begin{array}{ll}
z_{1 ; y, z}=\mathfrak{N}_{+}^{(0, \pi, \pi)}+\mathfrak{N}_{+}^{(\pi, \pi, \pi)} & \bmod 2, \\
z_{1 ; ;, z}=\mathfrak{N}_{+}^{(\pi, 0, \pi)}+\mathfrak{N}_{+}^{(\pi, \pi, \pi)} & \bmod 2
\end{array}
$$

and the factor $\mathbb{Z}_{4}$ corresponds to stacks of two-dimensional topological superconductors in the $z$ direction with indicator

$$
z_{2 ; z}=\sum_{\mathbf{k}_{s} \mid k_{s, z}=\pi} \mathfrak{N}_{+}^{\mathbf{k}_{s}}(-1)^{\left(k_{s, x}+k_{s, y}\right) / \pi} \bmod 4
$$

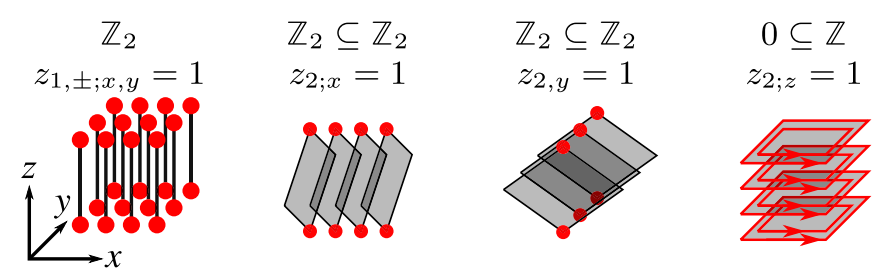

FIG. 13. Topological phases of a three-dimensional superconductor in tenfold-way class $\mathrm{D}$ with point group $C_{2}$ and representation $\Theta=B$.

The value $z_{2 ; z}=2$ is ambiguous since it may either correspond to a stack of first-order two-dimensional superconductors with Chern number two or to a stack of second-order two-dimensional superconductors (see Fig. 12). Our result (A3) for the group of symmetry-based indicators agrees with the corresponding result from [67].

\section{b. Representation $\Theta=B$}

Classifying group:

$$
\mathcal{K}_{\mathrm{D}}\left[C_{2}, B\right]=\mathbb{Z}_{2}^{4} \times \mathbb{Z} .
$$

A factor $\mathbb{Z}_{2}^{2}$ corresponds to one-dimensional superconductors parallel to the rotation (z) axis, stacked in the $x$ and $y$ directions, and with rotation parities \pm [labels $(1, \pm ; x, y)$ ]. A factor $\mathbb{Z}_{2}^{2}$ corresponds to two-dimensional second-order topological superconductors stacked in the $x$ or $y$ directions [labels $(2 ; x)^{\prime}$ and $\left.(2 ; y)^{\prime}\right]$. The remaining factor $\mathbb{Z}$ describes a two-dimensional even-Chern-number superconductor stacked in the $z$ direction with label $(2 ; z)$. They can be adiabatically deformed to normal-state Chern insulators. The boundary signatures of the topological phases together with their symmetry-based indicators are displayed in Fig. 13.

Band labels. There are $\mathrm{BL} \simeq \mathbb{Z}_{2}^{16}$ topological band labels given by the Pfaffian invariants $\mathfrak{p}_{ \pm}^{\mathbf{k}_{s}}$ in both even and odd rotation parity subspaces at all high-symmetry momenta $\mathbf{k}_{\mathrm{s}}$.

Compatibility relations. From the fact that $\mathcal{R}_{\pi} \mathcal{P}$ is a local symmetry in reciprocal space in high-symmetry planes, we derive the compatibility relation

$$
\begin{aligned}
\sum_{s= \pm} \mathfrak{p}_{s}^{\left(0,0, k_{z}\right)} & =\sum_{s= \pm} \mathfrak{p}_{s}^{\left(\pi, 0, k_{z}\right)} \\
& =\sum_{s= \pm} \mathfrak{p}_{s}^{\left(0, \pi, k_{z}\right)} \\
& =\sum_{s= \pm} \mathfrak{p}_{s}^{\left(\pi, \pi, k_{z}\right)} \text { for } k_{z}=0, \pi
\end{aligned}
$$

The compatibility relations identify a factor $\mathbb{Z}_{2}^{6}$ of $\mathrm{BL}$ as representation-enforced gapless nodal-line superconductors such that $\mathrm{BS} \simeq \mathbb{Z}_{2}^{10}$ and

$$
\begin{aligned}
B[H(\mathbf{k})]= & \left\{\mathfrak{p}_{-}^{(0,0,0)}, \mathfrak{p}_{-}^{(\pi, 0,0)}, \mathfrak{p}_{-}^{(0, \pi, 0)}, \mathfrak{p}_{+}^{(\pi, \pi, 0)}, \mathfrak{p}_{-}^{(\pi, \pi, 0)},\right. \\
& \left.\mathfrak{p}_{-}^{(0,0, \pi)}, \mathfrak{p}_{-}^{(\pi, 0, \pi)}, \mathfrak{p}_{-}^{(0, \pi, \pi)}, \mathfrak{p}_{+}^{(\pi, \pi, \pi)}, \mathfrak{p}_{-}^{(\pi, \pi, \pi)}\right\} .
\end{aligned}
$$

A second compatibility relation for gapped phases, identical to Eq. (98) in the main text, follows by considering Chern numbers at two-dimensional cuts through the Brillouin zone. 

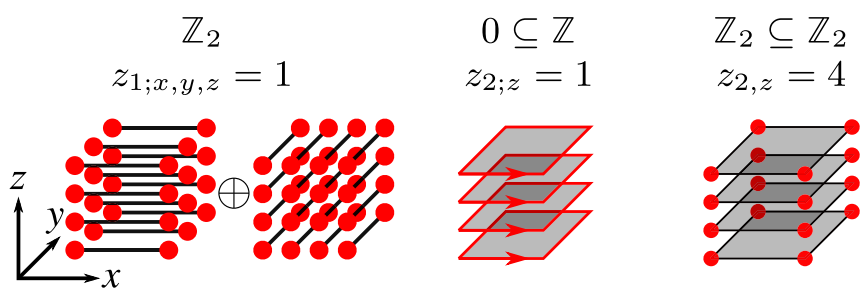

FIG. 14. Topological phases of a three-dimensional superconductor in tenfold-way class $\mathrm{D}$ with point group $C_{4}$ and representation $\Theta=A$ or $B$

Symmetry-based indicators. The group of symmetry-based indicators is

$$
\mathrm{SI}_{\mathrm{D}}\left[C_{2}, B\right] \simeq \mathbb{Z}_{2}^{6},
$$

out of which a factor $\mathbb{Z}_{2}^{5}$ corresponds to gapped topological phases. For phases corresponding to stacks of Kitaev chains we have

$$
z_{1, \pm ; x, y}=\mathfrak{p}_{ \pm}^{(\pi, \pi, \pi)}+\mathfrak{p}_{ \pm}^{(\pi, \pi, 0)} \bmod 2
$$

and for phases corresponding to stacks of two-dimensional superconductors in the $l=x, y, z$ direction we have

$$
z_{(2 ; l)}=\sum_{\mathbf{k}_{\mathrm{s}} \mid k_{\mathrm{s}, l}=\pi} \mathfrak{p}_{+}^{\mathbf{k}_{\mathrm{s}}}=\sum_{\left.\mathbf{k}_{\mathrm{s}}\right|_{\mathrm{s}, l}=\pi} \mathfrak{p}_{-}^{\mathbf{k}_{\mathrm{s}}} \bmod 2
$$

where the equality follows from the 0D compatibility relation. The remaining factor $\mathbb{Z}_{2}$ corresponds to a symmetry-based indicator for a nodal superconductor with different Chernnumber parity detected by the band labels,

$$
z_{3}=\sum_{\mathbf{k}_{\mathrm{s}}} \mathfrak{p}_{+}^{\mathbf{k}_{\mathrm{s}}}=\sum_{\mathbf{k}_{\mathrm{s}}} \mathfrak{p}_{-}^{\mathbf{k}_{\mathrm{s}}} \bmod 2 .
$$

The group (A4) of symmetry-based indicators differs from the result obtained in Ref. [67], where no symmetry-based indicators are found due to the absence of Pfaffian band labels.

\section{Point group $C_{4}$, class D \\ a. Representation $\Theta=A$ or $B$}

\section{Classifying group:}

$$
\mathcal{K}_{\mathrm{D}}\left[C_{4}, A\right]=\mathcal{K}_{\mathrm{D}}\left[C_{4}, B\right]=\mathbb{Z}_{2}^{2} \times \mathbb{Z} .
$$

A factor $\mathbb{Z}_{2}$ corresponds to Kitaev chains aligned perpendicular to the rotation axis and to each other and stacked in all three spatial directions [label $(1 ; x, y, z)]$. The remaining factor $\mathbb{Z}_{2} \times \mathbb{Z}$ corresponds to topological superconductors stacked perpendicular to the rotation axis [label $(2, z)]$, where the factor $\mathbb{Z}_{2}$ describes a second-order phase and the factor $\mathbb{Z}$ a Chern superconductor. Even-Chern-number superconductors can be adiabatically deformed to Chern insulators with vanishing superconducting correlations. The boundary signatures of the topological phases together with their symmetry-based indicators are displayed in Fig. 14.

Band labels. There are $\mathrm{BL} \simeq \mathbb{Z}^{10}$ topological band labels given by the invariants $\mathfrak{N}_{j}^{\mathbf{k}_{\mathrm{s}}}$ with $j=\frac{1}{2}, \frac{5}{2}$ at high-symmetry momenta $\mathbf{k}_{\mathrm{s}}$ with fourfold rotation symmetry and $j=\frac{1}{2}$ at high-symmetry momenta $\mathbf{k}_{\mathrm{s}}$ with twofold rotation symmetry.
Compatibility relations. For high-symmetry lines parallel to the rotation axis the invariant $\mathfrak{N}_{j}^{\mathbf{k}}$ is preserved for gapped phases, which gives the compatibility relation

$$
\mathfrak{N}_{j}^{\left(k_{x}^{\prime}, k_{y}^{\prime}, 0\right)}=\mathfrak{N}_{j}^{\left(k_{x}^{\prime}, k_{y}^{\prime}, \pi\right)} \text { for } k_{x}^{\prime}, k_{y}^{\prime}=0, \pi .
$$

This identifies a factor $\mathbb{Z}^{5}$ of BL as representation-enforced gapless superconductors with nodal points on high-symmetry lines in the Brillouin zone. We find that $\mathrm{BS} \simeq \mathbb{Z}^{5}$ and choose the independent band labels as

$$
B[H(\mathbf{k})]=\left\{\mathfrak{N}_{\frac{1}{2}}^{(0,0, \pi)}, \mathfrak{N}_{\frac{5}{2}}^{(0,0, \pi)}, \mathfrak{N}_{\frac{1}{2}}^{(\pi, 0, \pi)}, \mathfrak{N}_{\frac{1}{2}}^{(\pi, \pi, \pi)}, \mathfrak{N}_{\frac{5}{2}}^{(\pi, \pi, \pi)}\right\} .
$$

Symmetry-based indicators. The group of symmetry-based indicators is

$$
\mathrm{SI}_{\mathrm{D}}\left[C_{4}, A\right]=\mathrm{SI}_{\mathrm{D}}\left[C_{4}, B\right] \simeq \mathbb{Z}_{2} \times \mathbb{Z}_{8},
$$

isomorphic to the result of the two-dimensional plane discussed in Sec. VIB. The symmetry-based indicators for the stack of Kitaev chains $z_{1 ; x, y, z}$, two-dimensional second-order and Chern superconductors $z_{2 ; z}$ are identical to the symmetrybased indicators of the two-dimensional plane, Eqs. (85) and (86), respectively. As in the two-dimensional case, the weak second-order phase and the weak phase with Chern number 4 have identical band labels. In three dimensions, the symmetrybased indicators are expressed as

$$
\begin{aligned}
z_{1 ; x, y, z}= & \mathfrak{N}_{\frac{1}{2}}^{(0,0, \pi)}+\mathfrak{N}_{\frac{5}{2}}^{(0,0, \pi)}+\mathfrak{N}_{\frac{1}{2}}^{(\pi, 0, \pi)} \bmod 2, \\
z_{2 ; z}= & -\mathfrak{N}_{\frac{1}{2}}^{(0,0, \pi)}+3 \mathfrak{N}_{\frac{5}{2}}^{(0,0, \pi)}-2 \mathfrak{N}_{\frac{1}{2}}^{(\pi, 0, \pi)} \\
& +3 \mathfrak{N}_{\frac{1}{2}}^{(\pi, \pi, \pi)}-\mathfrak{N}_{\frac{5}{2}}^{(\pi, \pi, \pi)} \bmod 8 .
\end{aligned}
$$

The group of symmetry-based indicators (A5) is identical with the result from [67].

$$
\text { b. Representation } \Theta={ }^{1} E \text { or }{ }^{2} E
$$

Classifying group:

$$
\mathcal{K}_{\mathrm{D}}\left[C_{4},{ }^{2} E\right]=\mathcal{K}_{\mathrm{D}}\left[C_{4},{ }^{1} E\right]=\mathbb{Z}_{2}^{3} \times \mathbb{Z} .
$$

A factor $\mathbb{Z}_{2}^{2}$ corresponds to one-dimensional topological superconductors parallel to the rotation axis $(z)$ in the angular momentum subspaces $j=\frac{1}{2}, \frac{5}{2}$ [labels $(1, j ; x, y)$ ]. A factor $\mathbb{Z}$ corresponds to Chern superconductors with even Chern number stacked perpendicular to the rotation axis [label $(2, z)]$. These phases can be adiabatically deformed to Chern insulators with vanishing superconducting correlations. The remaining factor $\mathbb{Z}_{2}$ corresponds to a pair of two-dimensional second-order topological superconductors parallel to the rotation axis, exchanged by fourfold rotation and stacked in both $x$ and $y$ directions [label $(2 ; x, y)]$. The boundary signatures of the topological phases together with their symmetry-based indicators are displayed in Fig. 15.

Band labels. There are $\mathrm{BL} \simeq \mathbb{Z}_{2}^{12} \times \mathbb{Z}^{4}$ topological band labels given by the invariants $\mathfrak{N}_{3 / 2}^{\mathbf{k}_{\mathrm{s}}}, \mathfrak{p}_{1 / 2}^{\mathbf{k}_{\mathrm{s}}}, \mathfrak{p}_{5 / 2}^{\mathbf{k}_{\mathrm{s}}}$ at fourfold rotation-symmetric high-symmetry momenta $\mathbf{k}_{\mathrm{s}}=(0,0,0)$, $(0,0, \pi),(\pi, \pi, 0)$, and $(\pi, \pi, \pi)$, and $\mathfrak{p}_{1 / 2}^{\mathbf{k}_{\mathrm{s}}}, \mathfrak{p}_{3 / 2}^{\mathbf{k}_{\mathrm{s}}}$ at twofold rotation-symmetric high-symmetry momenta $\mathbf{k}_{\mathrm{s}}=(0, \pi, 0)$ and $(0, \pi, \pi)$.

Compatibility relations. From the fact that $\mathcal{R}_{\pi} \mathcal{P}$ is a local symmetry for high-symmetry planes in the Brillouin zone one 


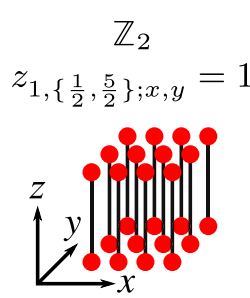

$$
\begin{gathered}
0 \subseteq \mathbb{Z} \\
z_{2 ; z}=1
\end{gathered}
$$

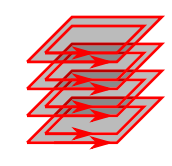

$$
\begin{gathered}
\mathbb{Z}_{2} \subseteq \mathbb{Z}_{2} \\
z_{2 ; x, y}=1
\end{gathered}
$$

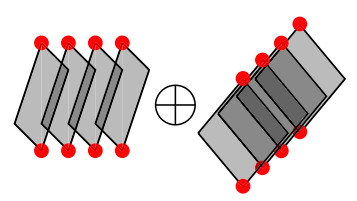

FIG. 15. Topological phases of a three-dimensional superconductor in tenfold-way class $\mathrm{D}$ with point group $C_{4}$ and representation $\Theta={ }^{1} E$ or ${ }^{2} E$.

derives the compatibility relation (87) of the main text. From the fact that $\mathfrak{N}_{j}$ is conserved along the fourfold symmetric lines in the Brillouin zone parallel to the rotation axis for $j=$ $\frac{3}{2}, \frac{7}{2}$ one derives the further compatibility relations

$$
\mathfrak{N}_{\frac{3}{2}}^{\left(k_{x}^{\prime}, k_{y}^{\prime}, 0\right)}=\mathfrak{N}_{\frac{3}{2}}^{\left(k_{x}^{\prime}, k_{y}^{\prime}, \pi\right)}
$$

for $\left(k_{x}^{\prime}, k_{y}^{\prime}\right)=(0,0),(\pi, \pi)$. The above compatibility relations associate factors $\mathbb{Z}_{2}^{4}$ and $\mathbb{Z}^{2}$ in BL with representationenforced gapless superconductors with nodal-line and nodal points, respectively. Hence, we have $\mathrm{BS} \simeq \mathbb{Z}_{2}^{8} \times \mathbb{Z}^{2}$ with

$$
\begin{aligned}
B[H(\mathbf{k})]= & \left\{\mathfrak{p}_{\frac{1}{2}}^{(0,0,0)}, \mathfrak{p}_{\frac{5}{2}}^{(0,0,0)}, \mathfrak{p}_{\frac{3}{2}}^{(\pi, 0,0)}, \mathfrak{p}_{\frac{5}{2}}^{(\pi, \pi, 0)}, \mathfrak{p}_{\frac{1}{2}}^{(0,0, \pi)},\right. \\
& \left.\mathfrak{p}_{\frac{5}{2}}^{(0,0, \pi)}, \mathfrak{N}_{\frac{3}{2}}^{(0,0, \pi)}, \mathfrak{p}_{\frac{3}{2}}^{(\pi, 0, \pi)}, \mathfrak{p}_{\frac{5}{2}}^{(\pi, \pi, \pi)}, \mathfrak{N}_{\frac{3}{2}}^{(\pi, \pi, \pi)}\right\} .
\end{aligned}
$$

A third compatibility relation for gapped phases follows by considering Chern numbers at two-dimensional cuts through the Brillouin zone:

$$
\begin{aligned}
& -\mathfrak{N}_{\frac{3}{2}}^{(0,0,0)}-\mathfrak{N}_{\frac{3}{2}}^{(\pi, \pi, 0)}+2 \mathfrak{p}_{\frac{5}{2}}^{(0,0,0)}+2 \mathfrak{p}_{\frac{3}{2}}^{(\pi, 0,0)}+2 \mathfrak{p}_{\frac{5}{2}}^{(\pi, \pi, 0)} \\
& =-\mathfrak{N}_{\frac{3}{2}}^{(0,0, \pi)}-\mathfrak{N}_{\frac{3}{2}}^{(\pi, \pi, \pi)}+2 \mathfrak{p}_{\frac{5}{2}}^{(0,0, \pi)} \\
& \quad+2 \mathfrak{p}_{\frac{3}{2}}^{(\pi, 0, \pi)}+2 \mathfrak{p}_{\frac{5}{2}}^{(\pi, \pi, \pi)} \bmod 4
\end{aligned}
$$

Symmetry-based indicators. The group of symmetry-based indicators is

$$
\mathrm{SI}_{\mathrm{D}}\left[C_{4},{ }^{1} E\right]=\mathrm{SI}_{\mathrm{D}}\left[C_{4},{ }^{2} E\right] \simeq \mathbb{Z}_{2}^{4} \times \mathbb{Z}_{4},
$$

out of which two factors $\mathbb{Z}_{2}$ correspond to stacks of Kitaev chains with indicator

$$
z_{1, j ; x, y}=\mathfrak{p}_{j}^{(\pi, \pi, 0)}+\mathfrak{p}_{j}^{(\pi, \pi, \pi)} \bmod 2
$$

for $j=\frac{1}{2}, \frac{5}{2}$, the factor $\mathbb{Z}_{4}$ corresponds to a stack of Chern superconductors perpendicular to the rotation axis $z_{2 ; z}$ with indicator given by

$$
\begin{aligned}
z_{2 ; z}= & -\mathfrak{N}_{\frac{3}{2}}^{(0,0, \pi)}-\mathfrak{N}_{\frac{3}{2}}^{(\pi, \pi, \pi)}+2 \mathfrak{p}_{\frac{5}{2}}^{(0,0, \pi)} \\
& +2 \mathfrak{p}_{\frac{3}{2}}^{(\pi, 0, \pi)}+2 \mathfrak{p}_{\frac{5}{2}}^{(\pi, \pi, \pi)} \bmod 4,
\end{aligned}
$$

and a factor $\mathbb{Z}_{2}$ corresponds to a stack of second-order Chern superconductors parallel to the rotation axis with symmetrybased indicator

$$
z_{2 ; x, y}=\mathfrak{p}_{\frac{3}{2}}^{(\pi, 0,0)}+\mathfrak{p}_{\frac{3}{2}}^{(\pi, 0, \pi)} \bmod 2 .
$$

The remaining factor $\mathbb{Z}_{2}$ corresponds to a nodal superconductor the Chern number of which changes by $4 n+2$ between

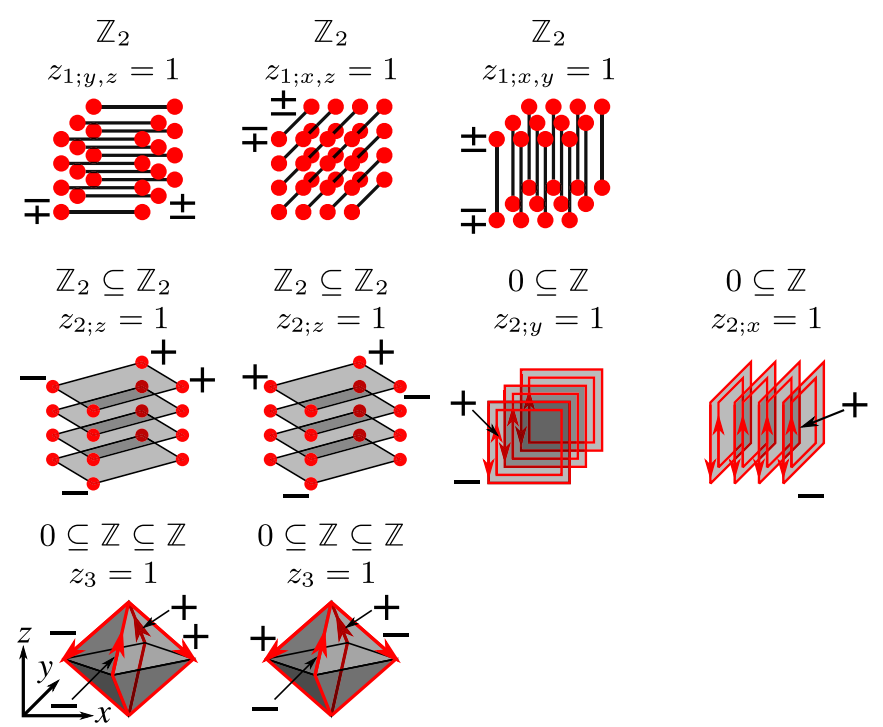

FIG. 16. Topological phases of a three-dimensional superconductor in tenfold-way class $\mathrm{D}$ with point group $C_{2 v}$ and representation $\Theta=A_{2}$. The parity of the gapless states under corresponding mirror symmetry is denoted by \pm .

parallel planes, with the difference detected by the band labels as

$$
z_{3}=\sum_{k_{z}=0, \pi} 2 \mathfrak{p}_{\frac{5}{2}}^{\left(0,0, k_{z}\right)}+2 \mathfrak{p}_{\frac{3}{2}}^{\left(\pi, 0, k_{z}\right)}+2 \mathfrak{p}_{\frac{5}{2}}^{\left(\pi, \pi, k_{z}\right)} \bmod 4
$$

Note that a change of the Chern number by an odd number is prohibited by the zero-dimensional compatibility constraint along rotation lines.

Reference [67] finds the group $\mathrm{SI}=\mathbb{Z}_{2}$. Upon omitting band labels corresponding to Pfaffians in our discussion, this single factor $\mathbb{Z}_{2}$ is identified as the parity of $z_{2 ; z}$, the Chern number of a stack of two-dimensional topological superconductors in parallel to the rotation axis.

\section{Point group $C_{2 v}$, class $\mathrm{D}$}

The classification and symmetry labels are trivial for representation $\Theta=A_{1}$. The $B_{1}$ representation is analogous to the $B_{2}$ representation by exchanging the labels $x, y$.

For the $A_{1}$ representation, our results agree with those of Ref. [67]. For the other representations $A_{2}, B_{1}, B_{2}$, the band labels are defined exclusively in terms of Pfaffians. Correspondingly, in Ref. [67], no symmetry-based indicators are found due to the absence of Pfaffian band labels in their construction.

\section{a. Representation $\Theta=A_{2}$}

\section{Classifying group:}

$$
\mathcal{K}_{\mathrm{D}}\left[C_{2 v}, A_{2}\right]=\mathbb{Z}_{2}^{5} \times \mathbb{Z}^{4}
$$

(see Appendix B 6 for a derivation and for the definition of the labels). The boundary signatures of the topological phases together with their symmetry-based indicators are displayed in Fig. 16. 
Band labels. There are $\mathrm{BL} \simeq \mathbb{Z}_{2}^{8}$ topological band labels given by the Pfaffian invariant $\mathfrak{p}^{\mathbf{k}_{\mathbf{s}}}$ at all high-symmetry momenta $\mathbf{k}_{\mathrm{s}}$.

Compatibility relations. There are no compatibility relations restricting the symmetry-based indicators for gapped phases. Thus, $\mathrm{BS}=\mathrm{BL}$ and

$$
B[H(\mathbf{k})]=\left\{\mathfrak{p}^{\mathbf{k}_{\mathrm{s}}}\right\} .
$$

Symmetry-based indicators. The group of symmetry-based indicators is

$$
\mathrm{SI}_{\mathrm{D}}\left[C_{2 v}, A_{2}\right] \simeq \mathbb{Z}_{2}^{7} .
$$

The symmetry-based indicators of the stacks of onedimensional topological superconductors with stacking directions $(i, j)=(y, z),(x, z),(x, y)$ are

$$
z_{1 ; i, j}=\sum_{\mathbf{k}_{\mathrm{s}} k_{k_{s, i}=k_{s, j}=\pi}} \mathfrak{p}^{\mathbf{k}_{\mathrm{s}}} \bmod 2,
$$

and for the stacks of two-dimensional topological superconductors with stacking directions $l=x, y, z$ they are

$$
z_{2, l}=\sum_{\mathbf{k}_{s} \mid k_{\mathrm{s}, l}=\pi} \mathfrak{p}^{\mathbf{k}_{\mathrm{s}}} \bmod 2,
$$

and for the three-dimensional second-order topological superconductors the symmetry-based indicator is

$$
z_{3}=\sum_{\mathbf{k}_{\mathrm{s}}} \mathfrak{p}^{\mathbf{k}_{\mathrm{s}}} \bmod 2 .
$$

\section{b. Representation $\Theta=B_{2}$}

Classifying group:

$$
\mathcal{K}_{\mathrm{D}}\left[C_{2 v}, B_{2}\right]=\mathbb{Z}_{2}^{3} \times \mathbb{Z}^{2}
$$

(see Appendix B 6 for a derivation and for the definition of the labels). The boundary signatures of the topological phases together with their symmetry-based indicators are displayed in Fig. 17.

Band labels. There are $\mathrm{BL} \simeq \mathbb{Z}_{2}^{8}$ topological band labels given by the Pfaffian invariant $\mathfrak{p}^{\mathbf{k}_{\mathrm{s}}}$ at all high-symmetry momenta $\mathbf{k}_{\mathrm{s}}$.

Compatibility relations. From the fact that $\mathcal{P} M_{x}$ acts locally on high-symmetry lines in reciprocal space we derive the compatibility relation

$$
\mathfrak{p}^{\left(0, k_{y}^{\prime}, k_{z}^{\prime}\right)}=\mathfrak{p}^{\left(\pi, k_{y}^{\prime}, k_{z}^{\prime}\right)} \text { for } k_{y}^{\prime}, k_{z}^{\prime}=0, \pi .
$$

The compatibility relations identify a factor $\mathbb{Z}_{2}^{4}$ of BL as representation-enforced gapless superconductors with nodal points such that $\mathrm{BS} \simeq \mathbb{Z}_{2}^{4}$ and

$$
B[H(\mathbf{k})]=\left\{\mathfrak{p}^{\mathbf{k}_{\mathrm{s}} \mid k_{x}=\pi}\right\} .
$$

Symmetry-based indicators. The group of symmetry-based indicators is

$$
\mathrm{SI}_{\mathrm{D}}\left[C_{2 v}, B_{2}\right] \simeq \mathbb{Z}_{2}^{3} .
$$

The symmetry-based indicators of the stacks of onedimensional topological superconductors with stacking direc-
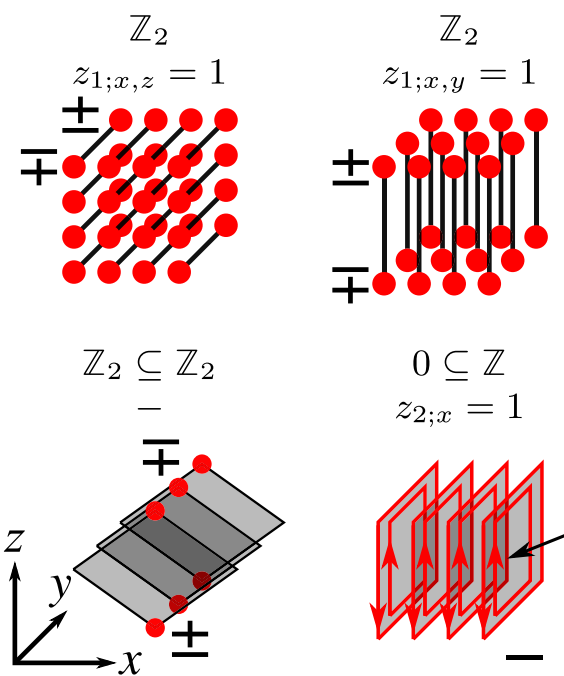

$$
\begin{gathered}
0 \subseteq \mathbb{Z} \\
z_{2 ; x}=1
\end{gathered}
$$$$
0 \subseteq \mathbb{Z} \subseteq \mathbb{Z}
$$
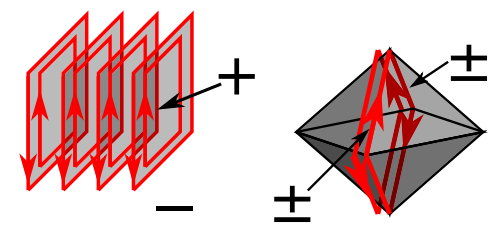

FIG. 17. Topological phases of a three-dimensional superconductor in tenfold-way class D with point group $C_{2 v}$ and representation $\Theta=B_{2}$. The parity of the gapless states under corresponding mirror symmetry is denoted by \pm .

tions $(i, j)=(x, z),(x, y)$ are

$$
z_{1 ; i, j}=\sum_{\mathbf{k}_{\mathrm{s}} \mid k_{\mathrm{s}, i}=k_{\mathrm{s}, j}=\pi} \mathfrak{p}^{\mathbf{k}_{\mathrm{s}}} \bmod 2,
$$

and for the stacks of two-dimensional topological superconductors with stacking direction $x$ it is

$$
z_{2, x}=\sum_{\left.\mathbf{k}_{\mathrm{s}}\right|_{k_{s, x}=\pi}} \mathfrak{p}^{\mathbf{k}_{\mathrm{s}}} \bmod 2 .
$$

The two-dimensional second-order topological superconductor stacked in the $y$ direction and the three-dimensional second-order topological superconductor can not be detected from symmetry-based indicators.

\section{APPENDIX B: BOUNDARY CLASSIFICATION GROUPS $\mathcal{K}$}

For tenfold-way classes in $d$ dimensions, the classification groups $\mathcal{K}$ follow from the "periodic table of topological phases" $[9,10]$. They are the direct product of groups classifying strong phases and groups classifying weak phases obtained by stacking topological phases in dimension $d-n$, $n=1, \ldots, d-1$.

In the presence of an additional order-two crystalline symmetry, such as inversion, mirror, or twofold rotation, the classifying group $\mathcal{K}$ can be obtained using the known classifications of strong phases given in Refs. [36,39] (see also Refs. $[46,48,80]$ ), again accounting for weak phases by taking direct products of the appropriate classification groups in lower dimensions [36]. It is important to point out that the classification group $\mathcal{K}$ we consider in this paper classifies topological phases with nontrivial boundary signature only (see the discussion at the end of Sec. III). These "boundary classification groups" $\mathcal{K}$ are obtained from the groups $K$ classifying the bulk band structure by dividing out the classifying group $K^{(d)}$ of atomic-limit phases [39].

Classifications of strong tenfold-way phases with other additional crystalline symmetry groups can be found in the 
literature [40,41]. We here give classification results for the examples discussed in the main text and in Appendix A. These classifications are derived from the enumeration of the possible anomalous boundary states compatible with the symmetries defining the topological class. Boundary states that can be removed by a change of lattice termination are removed from the classification because they are not a consequence of the topology of the bulk band structure. (Such boundary states are called "extrinsic" in Refs. [39,70].)

Below we compute the classifying groups $\mathcal{K}_{\eta}\left[G \mid G_{\mathcal{O}}, \Theta, d\right]$ of $d$-dimensional topological phases with point group $G$, where its normal subgroup $G_{\mathcal{O}}$ is assumed to act as onsite symmetry. For each factor of $\mathcal{K}_{\eta}\left[G \mid G_{\mathcal{O}}, \Theta, d\right]$ we present an interpretation in terms of higher-order topological phases or stacks of lower-dimensional topological (weak) phases and generators from which the band labels can be computed. We not only consider the dimensions $d$ corresponding to the examples used in the main text, but also lower dimensions, since the classification results for lower dimensions $d$ provides information on weak and higher-order phases.

\section{One dimension, class $\mathrm{D}, C_{2 v} \mid C_{s}$}

We consider a one-dimensional system extended in the $x$ direction, such that it lies within the mirror plane of $\mathcal{M}_{y}$. Hence, $\mathcal{M}_{y}$ is an onsite symmetry, whereas $\mathcal{M}_{x}$ acts nonlocally. For spinful fermions there is a single irreducible representation $\alpha=\bar{E}$ with dimension $d_{\bar{E}}=2$.

Representation $\Theta=A_{1}$. For the representation $\Theta=A_{1}$, $\mathcal{M}_{x}$ and $\mathcal{M}_{y}$ commute with particle-hole conjugation $\mathcal{P}$. The parallel mirror plane $\mathcal{M}_{y}$ with $\mathcal{M}_{y}^{2}=-1$ forbids a topological superconducting phase, as it effectively turns the onedimensional system into class $\mathrm{A}^{\mathcal{P}^{-} \mathcal{M}}$ with trivial classification (see the notation of Ref. [39]). We conclude that

$$
\mathcal{K}_{\mathrm{D}}\left[C_{2 v} \mid C_{s}, A_{1}, d=1\right]=0 .
$$

Representation $\Theta=A_{2}$. In this case, $\mathcal{M}_{x}$ and $\mathcal{M}_{y}$ anticommute with $\mathcal{P}$. The parallel mirror plane allows to block diagonalize the system into two blocks according to $\mathcal{M}_{y}$ parity \pm . Particle-hole conjugation acts within the blocks, whereas the perpendicular mirror plane $\mathcal{M}_{x}$ interchanges the two blocks. The system is thus completely specified by a single block in tenfold-way class D, which allows a Kitaev-chain topological phase. A generator of the nontrivial phase is

$$
H_{(1)}\left(k_{x}\right)=\rho_{0} \tau_{3}\left(1-m-\cos k_{x}\right)+\rho_{1} \tau_{1} \sin k_{x},
$$

with $0<m<2$ and representations $\mathcal{P}=\rho_{0} \tau_{1} K, U\left(\mathcal{R}_{\pi}\right)=$ $i \rho_{2} \tau_{0}, U\left(\mathcal{M}_{x}\right)=i \rho_{3} \tau_{0}, U\left(\mathcal{M}_{y}\right)=i \rho_{1} \tau_{0}$.

Representation $\Theta=B_{1}$. In this case, $\mathcal{M}_{x}$ commutes with $\mathcal{P}$, whereas $\mathcal{M}_{y}$ anticommutes with $\mathcal{P}$. The discussion can be mapped to that of the case $\Theta=A_{2}$ by noting that $\mathcal{R}_{\pi}$ anticommutes with $\mathcal{P}$, so that the arguments put forward for $\Theta=A_{1}$ can be applied to the case $\Theta=B_{2}$ by exchanging the roles of $\mathcal{M}_{x}$ and $\mathcal{R}_{\pi}$. It follows that

$$
\mathcal{K}_{\mathrm{D}}\left[C_{2 v} \mid C_{s}, B_{1}, d=1\right]=\mathbb{Z}_{2} .
$$

Representation $\Theta=B_{2}$. The parallel mirror plane $\mathcal{M}_{y}$ with $\mathcal{M}_{y}^{2}=-1$ forbids a topological superconducting phase, as it effectively turns the one-dimensional system into class $\mathrm{A}^{\mathcal{P}^{+} \mathcal{M}}$ with trivial classification [39], so that

$$
\mathcal{K}_{\mathrm{D}}\left[C_{2 v} \mid C_{s}, B_{2}, d=1\right]=0 .
$$

\section{One dimension, class $\mathrm{D}, C_{2 v} \mid C_{2 v}$}

This case applies to a one-dimensional system along the $z$ direction, such that it lies in the intersection of the mirror planes $\mathcal{M}_{x}, \mathcal{M}_{y}$ and the full crystalline symmetry group $C_{2 v}$ acts onsite. As shown in Table $\mathrm{V}$ and discussed in Sec. III, for spinful fermions there is a single irreducible representation $\alpha=\bar{E}$ with dimension $d_{\bar{E}}=2$, which effectively changes the tenfold-way symmetries from those of class $\mathrm{D}$ to those of class C for the case $\Theta=A_{1}$, but leaves them unchanged for $\Theta=$ $A_{2}, B_{1}$, and $B_{2}$. It follows for $d=1$ that

$$
\begin{gathered}
\mathcal{K}_{\mathrm{D}}\left[C_{2 v} \mid C_{2 v}, A_{1}, d=1\right]=0, \\
\mathcal{K}_{\mathrm{D}}\left[C_{2 v} \mid C_{2 v}, \Theta, d=1\right]=\mathbb{Z}_{2}, \Theta=A_{2}, B_{1}, B_{2} .
\end{gathered}
$$

A generator for the nontrivial phases is

$$
H_{(1)}\left(k_{z}\right)=\rho_{0} \tau_{3}\left(1-m-\cos k_{z}\right)+\rho_{1} \tau_{1} \sin k_{z},
$$

with $0<m<2$ and accordingly chosen representations.

\section{Two dimensions, class $\mathrm{D}, C_{2 v} \mid C_{s}$}

This case applies to a two-dimensional system in the $x z$ plane, such that $\mathcal{M}_{y}$ acts as an onsite symmetry. A system with boundaries parallel to the coordinate axes has two $\mathcal{M}_{x^{-}}$ symmetric boundaries and two boundaries that are mapped to each other by $\mathcal{M}_{x}$.

Representation $\Theta=A_{1}$. From an analysis of the reflectionsymmetric boundary, we find that there is no first-order topological superconducting phase. (The nontrivial first-order phase in class D has a chiral edge mode, which is not compatible with a mirror symmetry.) There are also no second-order or weak phases in this representation,

$$
\mathcal{K}_{\mathrm{D}}\left[C_{2 v} \mid C_{s}, A_{1}, d=2\right]=0 .
$$

This follows from the triviality of the classifying groups $\mathcal{K}_{\mathrm{D}}\left[C_{2 v} \mid C_{s}, A_{1}, d\right]$ and $\mathcal{K}_{\mathrm{D}}\left[C_{2 v} \mid C_{2 v}, A_{1}, d\right]$ for $d=1$, into which an eventual higher-order phase can be deformed or which could be stacked to form a weak phase (see the discussions in Appendices B 1 and B 2).

Representation $\Theta=A_{2}$. A $\mathcal{M}_{x}$-symmetric boundary allows counterpropagating chiral Majorana modes in opposite $\mathcal{M}_{y}$-parity subspaces. They are the first-order boundary signature of a pair of Chern superconductors related to each other by $\mathcal{M}_{x}$. Superconductors with even Chern number in a mirror plane can be adiabatically deformed to Chern insulators with vanishing superconducting correlations. From the nontriviality of the classifying groups $\mathcal{K}_{\mathrm{D}}\left[C_{2 v} \mid C_{s}, A_{2}, d\right]=$ $\mathcal{K}_{\mathrm{D}}\left[C_{2 v} \mid C_{2 v}, A_{2}, d\right]=\mathbb{Z}_{2}$ for $d=1$ we conclude that two types of weak phases are possible, obtained by stacking one-dimensional superconductors in the $x$ and $z$ directions. There is no second-order phase, despite the fact that pairs of even- and odd- $\mathcal{M}_{y}$-parity Majorana zero-energy bound states are allowed at corners. Such states are protected against local perturbations at corners, but they can be removed by a change of termination along the crystal edges. (Formally, this follows because the group $\mathcal{K}_{\mathrm{D}}\left[C_{2 v} / C_{2 v}, A_{2}\right]$ classifying 
$\mathcal{M}_{x}$-symmetric corner states is "separable" in the language of Ref. [39].) We thus conclude that

$$
\mathcal{K}_{\mathrm{D}}\left[C_{2 v} \mid C_{s}, A_{2}, d=2\right]=\mathbb{Z}_{2}^{2} \times \mathbb{Z} .
$$

The same result can be obtained by arguing that the onsite symmetry $\mathcal{M}_{y}$ commutes with $\mathcal{P}$, so that the Hamiltonian can be written as the diagonal sum of $\mathcal{M}_{y}$-even and $\mathcal{M}_{y}$-odd blocks that each satisfy particle-hole symmetry. The mirror symmetry $\mathcal{M}_{x}$ interchanges the two blocks. Hence, the topological classification is the same as a two-dimensional Hamiltonian in tenfold-way class D without crystalline symmetries, which also gives Eq. (B7).

Representation $\Theta=B_{1}$. This case can be mapped to $\Theta=$ $A_{2}$ by interchanging the roles of $\mathcal{M}_{x}$ and $\mathcal{R}_{\pi}$. One thus finds

$$
\mathcal{K}_{\mathrm{D}}\left[C_{2 v} \mid C_{s}, B_{1}, d=2\right]=\mathbb{Z}_{2}^{2} \times \mathbb{Z} .
$$

Representation $\Theta=B_{2}$. In this case no first-order boundary signatures are allowed on a $\mathcal{M}_{x}$-symmetric boundary because they are incompatible with the $\mathcal{M}_{x}$ symmetry. To check for the existence of second-order or weak phases, we note that one-dimensional superconductors in the $z$ direction (i.e., perpendicular to the mirror plane of $\mathcal{M}_{x}$ ) have classifying group $\mathcal{K}_{\mathrm{D}}\left[C_{2 v} \mid C_{s}, B_{2}, d=1\right]$, which is trivial (see Appendix B 1). Also, one-dimensional superconductors in the $z$ direction, i.e., in the intersection of both mirror planes, have classifying group $\mathcal{K}_{\mathrm{D}}\left[C_{2 v} \mid C_{2 v}, B_{2}, d=1\right]=\mathbb{Z}_{2}$, as shown in Appendix B 2. This has two consequences: (i) A secondorder boundary signature corresponding to a pair of Majorana bound states at the $\mathcal{M}_{x}$-symmetric corner is possible. The in-plane mirror symmetry $\mathcal{M}_{y}$ with $\mathcal{M}_{y} \mathcal{P}=\mathcal{P} \mathcal{M}_{y}$ forbids one-dimensional topological phases within the mirror $\mathcal{M}_{y}$ plane that do not satisfy $\mathcal{M}_{x}$ mirror symmetry. Hence, the boundary signature can not be removed by a decoration of the crystal boundary and there is a second-order topological superconducting phase. A generator for this phase is

$$
\begin{aligned}
H_{2}^{\prime}(\mathbf{k})= & \mu_{0} \rho_{0} \tau_{3}\left(\cos k_{x}+\cos k_{z}+m\right) \\
& +\mu_{1} \rho_{0} \tau_{1} \sin k_{x}+\mu_{2} \rho_{2} \tau_{1} \sin k_{z},
\end{aligned}
$$

with $0<m<2$ and representations $\mathcal{P}=\mu_{0} \rho_{0} \tau_{1} K$, $U\left(\mathcal{R}_{\pi}\right)=i \mu_{0} \rho_{3} \tau_{3}, U\left(\mathcal{M}_{x}\right)=i \mu_{3} \rho_{3} \tau_{0}, U\left(\mathcal{M}_{y}\right)=i \mu_{3} \rho_{0} \tau_{3}$. Alternatively, the parallel mirror plane allows to block diagonalize the system into two blocks according to $\mathcal{M}_{y}$ parity \pm . Particle-hole conjugation interchanges the two blocks, and each block belongs to class $\mathrm{A}^{\mathcal{P}^{+} \mathcal{M}}$ with $0 \subseteq \mathbb{Z}_{2} \subseteq \mathbb{Z}_{2}$ bulk subgroup sequence for $d=2$ [39]. (ii) There is a weak phase corresponding to one-dimensional topological superconductors in the $z$ direction, which are stacked in the $x$ direction. We thus conclude that

$$
\mathcal{K}_{\mathrm{D}}\left[C_{2 v} \mid C_{s}, B_{2}, d=2\right]=\mathbb{Z}_{2}^{2} .
$$

\section{Two dimensions, class $\mathrm{D}, C_{2 v}$}

We now derive the boundary classification for the example discussed in Sec. VIA of the main text. The two mirror symmetries forbid a phase with a nonzero Chern number. Hence, there are no first-order phases for all representations of the pairing term $\Theta$.

Representation $\Theta=A_{1}$. For this representation, both $\mathcal{M}_{x}$ and $\mathcal{M}_{y}$ with $\mathcal{M}_{x}^{2}=\mathcal{M}_{y}^{2}=-1$ commute with $\mathcal{P}$, ruling (a)

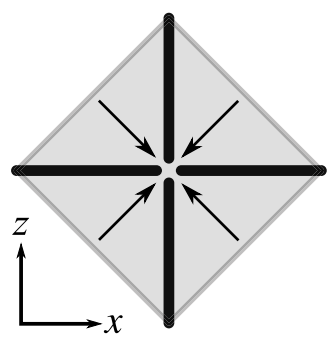

(b)

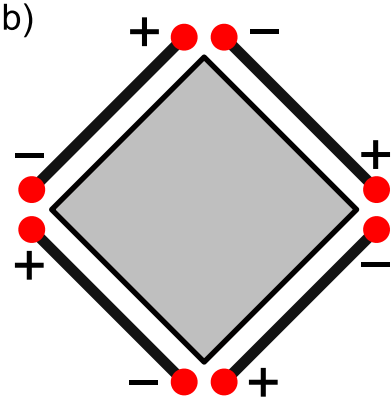

FIG. 18. (a) A $C_{2 v}$-symmetric two-dimensional crystal with two perpendicular mirror symmetries with gapped edges may be deformed into four one-dimensional chains in a crosslike arrangement. Corner states of the two-dimensional crystal are in one-to-one correspondence with end states of the one-dimensional structure. (b) A $C_{2 v}$-symmetric crystal may be decorated symmetrically with Kitaev chains, resulting in the appearance of extrinsic pairs of corner states at all four mirror-symmetric corners.

out the existence of zero-energy Majorana bound states at mirror-symmetric corners. There are no weak phases as the classifying group $\mathcal{K}_{\mathrm{D}}\left[C_{2 v} \mid C_{s}, A_{1}, d=1\right]=0$ is trivial. We conclude that

$$
\mathcal{K}_{\mathrm{D}}\left[C_{2 v}, A_{1}, d=2\right]=0 .
$$

Representation $\Theta=A_{2}$. In this case, both $\mathcal{M}_{x}$ and $\mathcal{M}_{y}$ anticommute with $\mathcal{P}$. We first investigate the possibility of second-order phases with nondegenerate zero-energy Majorana corner states at mirror-symmetric corners of a $C_{2 v^{-}}$ symmetric crystal. Since the edges of such a crystal are gapped, it may be deformed into a "cross" shape consisting of four one-dimensional chains [see Fig. 18(a)]. Each chain has a parallel mirror plane, which effectively acts as a local symmetry. Perpendicular mirror planes connect chains on opposite sides of the cross, but no longer act inside a chain. The parallel mirror planes allow the Hamiltonian to be block diagonalized into blocks with odd and even mirror parity. Each of these blocks is in tenfold-way class D, thus in principle allowing for the existence of up to two zero-energy Majorana end states, which turn into corner states if the system is deformed back to a $C_{2 v}$-symmetric two-dimensional crystal. The presence of the perpendicular mirror planes and the condition that the center of the cross be gapped restrict the possible configurations of corner states: Opposite corners must have Majorana states of opposite mirror parities since the two mirror operations anticommute, and the total number of Majorana modes must be a multiple of four. This leaves a $\mathbb{Z}_{2}^{3}$ extrinsic classification of allowed Majorana corner modes. To obtain the classification of intrinsic second-order phases, we must divide out configurations of corner states that differ by a change of termination. Hereto we note that the four edges of a $C_{2 v}$-symmetric crystal allow a "decoration" with Kitaev chains, which yield opposite-parity pairs of Majorana states at all four corners of the crystal [see Fig. 18(b)], so that a $\mathbb{Z}_{2}^{2}$ classifying group of second-order phases remains. The generators of the two distinct $\mathbb{Z}_{2}$ second-order phases can be given as configurations with single zero-energy Majorana bound states at all corners of a $C_{2 v}$-symmetric sample with mirror parities as indicated in Table XI. 

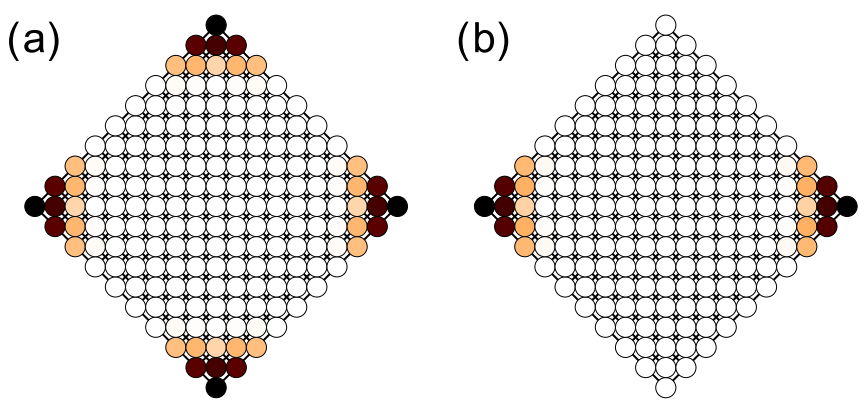

FIG. 19. (a) Support of Majorana bound states for the Hamiltonians $H_{2, \pm}^{\prime}$ of Eq. (B12). A symmetry-preserving next-nearestneighbor term $m_{1} \rho_{2} \tau_{1} \sin k_{x} \sin k_{y}$ with $m_{1}=0.4$ is added to remove a spurious gapless edge mode. (b) Support of Majorana bound states for $H_{2,+}^{\prime} \oplus H_{2,-}^{\prime}$, after adding an additional weak symmetrypreserving hybridization term $m_{2} \rho_{0} \tau_{1} \mu_{2}$ with strength $m_{2}=0.1$. The corner modes may appear at the other pair of corners if a different hybridization term is chosen.

Furthermore, there are two weak phases as the classifying group $\mathcal{K}_{\mathrm{D}}\left[C_{2 v} \mid C_{s}, A_{2}, d=1\right]=\mathbb{Z}_{2}$ (see Appendix B 1) allows stacking of one-dimensional topological superconductors both in $x$ and $y$ directions. Combining everything, we have the classifying group

$$
\mathcal{K}_{\mathrm{D}}\left[C_{2 v}, A_{2}, d=2\right]=\mathbb{Z}_{2}^{4} .
$$

Generators of the second-order phases are

$$
\begin{aligned}
H_{2, \pm}^{\prime}(\mathbf{k})= & \rho_{0} \tau_{3}\left(2-m-\cos k_{x}-\cos k_{y}\right) \\
& \pm \rho_{1} \tau_{1} \sin k_{x}+\rho_{3} \tau_{1} \sin k_{y}
\end{aligned}
$$

with $0<m<2$ and representations $\mathcal{P}=\tau_{1} K, U\left(\mathcal{R}_{\pi}\right)=$ $i \rho_{2} \tau_{0}, U\left(\mathcal{M}_{x}\right)=i \rho_{3} \tau_{0}, U\left(\mathcal{M}_{y}\right)=i \rho_{1} \tau_{0}$. To verify that these are indeed second-order phases with the desired properties, one may either count the number crystalline symmetrybreaking mass terms [39], or simply verify that these model Hamiltonians have the correct corner-state structure. Results of such a calculation, using the KWANT software [83] are shown in Fig. 19(a). As shown in Fig. 19(b), taking the direct sum of and hybridizing the two generating Hamiltonians $H_{2, \pm}^{\prime}$ yields a pair of Majorana bound states with opposite mirror parity at a single pair corners within a single mirror plane only.

Representations $\Theta=B_{1,2}$. The discussions for the cases $\Theta=B_{1}$ and $B_{2}$ are analogous, as they are related by a $\pi / 2$ rotation of the system. In the following we focus on the $B_{2}$ case, for which $\mathcal{M}_{x}$ anticommutes with $\mathcal{P}$ and $\mathcal{M}_{y}$ commutes with $\mathcal{P}$. As before, we first consider the possibility of second-order phases with zero-energy Majorana corner states. Only corners bisected by the mirror plane $\mathcal{M}_{x}$ can host Majorana bound states, as corners bisected by $\mathcal{M}_{y}$ are effectively in class A. Hence, the system can be deformed to a one-dimensional system within the mirror plane $\mathcal{M}_{x}$. This one-dimensional system has classifying group $\mathcal{K}_{\mathrm{D}}\left[C_{2 v} \mid C_{s}, B_{1}, d=1\right]=\mathbb{Z}_{2}$, as discussed in Appendix B 1. However, the corresponding configuration of boundary states, a pair of Majorana bound states in both mirror parity sectors at both corners bisected by the mirror $\mathcal{M}_{x}$ plane, can be removed by a decoration with Kitaev chains on each surface of a symmetric sample. We thus

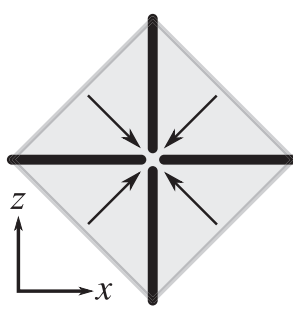

FIG. 20. A $C_{4}$-symmetric two-dimensional crystal may be deformed into four one-dimensional chains in a crosslike arrangement. Corner states of the two-dimensional crystal are in one-to-one correspondence with end states of the one-dimensional structure.

conclude that there are no intrinsic second-order phases for $\Theta=B_{1}$ or $B_{2}$.

Weak phases can be obtained by stacking one-dimensional chains in the $y$ direction. Since $\mathcal{M}_{x}$ is an onsite symmetry for such chains, their classification is given by the onedimensional classifying group $\mathcal{K}_{\mathrm{D}}\left[C_{2 v} \mid C_{s}, B_{1}, d=1\right]=\mathbb{Z}_{2}$ (see Appendix B 1). (Note that for this stacking direction $\mathcal{M}_{x}$ and $\mathcal{M}_{y}$ are exchanged in comparison to the discussion in Appendix B 1.) Superconductors in the perpendicular stacking direction $x$ are trivial since $\mathcal{K}_{\mathrm{D}}\left[C_{2 v} \mid C_{s}, B_{2}, d=1\right]=0$, as shown in Appendix B 1. We conclude that

$$
\mathcal{K}_{\mathrm{D}}\left[C_{2 v}, B_{1,2}, d=2\right]=\mathbb{Z}_{2} \text {. }
$$

\section{Two dimensions, class $\mathrm{D}, C_{4}$}

Representations $\Theta=A, B$. The representations $\Theta=A$ and $B$ have the same algebraic structure, so that we may limit the discussion to the case $\Theta=A$. This case allows a strong firstorder phase with a single chiral Majorana mode, corresponding to $\mathrm{a} \mathbb{Z}$ topological classification. To check for higher-order phases with four Majorana bound states, we smoothly deform the two-dimensional system to four one-dimensional chains arranged in a $C_{4}$-symmetric crosslike shape, as in Fig. 20. Each chain may harbor a zero-energy Majorana bound state at its end. The end states at the four chains are related to each other by $C_{4}$ symmetry. Since classification of zeroenergy Majorana modes protected by $C_{4}$ is trivial for $\mathrm{A}$ and B superconducting pairing (see discussion in Sec. III), the four Majorana states that would appear at the center of the cross can gap out, so that one obtains a true second-order phase with a $\mathbb{Z}_{2}$ classification. Finally, a weak phase may be obtained by stacking one-dimensional $C_{2}$-symmetric $x$ lines (with representation $\Theta=A$ ) in the $y$ direction and superimposing the same stack, rotated by $\pi / 2$. The one-dimensional superconductors that are the building blocks of this phase have zero-energy Majorana states at their ends and a $\mathbb{Z}_{2}$ classification. We conclude that

$$
\begin{aligned}
\mathcal{K}_{\mathrm{D}}\left[C_{4}, A, d=2\right] & =\mathcal{K}_{\mathrm{D}}\left[C_{4}, B, d=2\right] \\
& =\mathbb{Z}_{2}^{2} \times \mathbb{Z} .
\end{aligned}
$$

Representations $\Theta={ }^{1,2} E$. The representations $\Theta={ }^{1} E$ and ${ }^{2} E$ allow a strong first-order phase with an even number of chiral Majorana modes. One verifies that a single Majorana mode is not allowed for this representation since there is no one-dimensional representation of $\mathcal{R}_{\pi}$ and $\mathcal{P}$ meeting the 
(a)

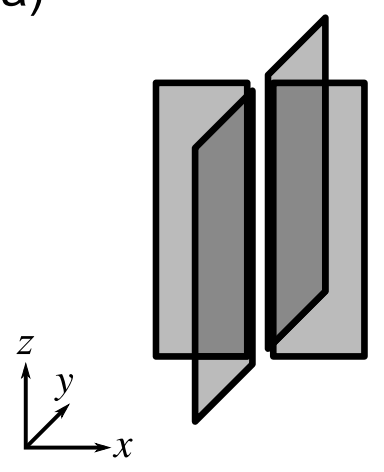

(b)

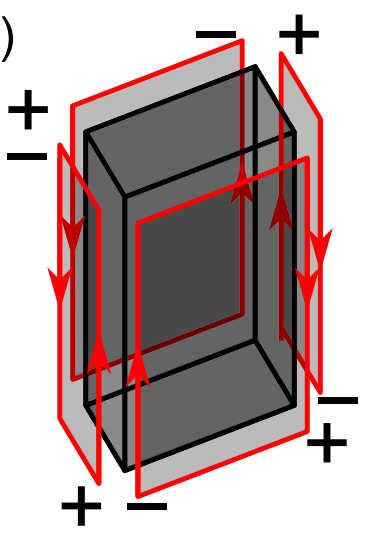

FIG. 21. (a) A $C_{2 v}$-symmetric three-dimensional crystal with two perpendicular mirror symmetries may be deformed into four twodimensional planes in a crosslike arrangement. Hinge states of the two-dimensional crystal are in one-to-one correspondence with edge states of the two-dimensional structure. (b) A $C_{2 v}$-symmetric crystal may be decorated symmetrically with quantum Hall planes, resulting in the appearance of extrinsic configurations of chiral hinge modes at all four mirror-symmetric hinges.

conditions that (i) $i \mathcal{R}_{\pi}$ squares to one and (ii) $i \mathcal{R}_{\pi}$ anticommutes with $\mathcal{P}$. An even number of Majorana modes are allowed since there are two-dimensional representations meeting these requirements. One also verifies that the constructions of a second-order phase and a weak phase used for the representations $\Theta=A, B$ discussed above do not work for the case $\Theta={ }^{1,2} E$. For the second-order phase, the reason is that at the center cross, where $C_{4}$ is a local symmetry, one now ends up with states at all four allowed angular momenta $j=$ $\frac{1}{2}, \frac{3}{2}, \frac{5}{2}$, and $\frac{7}{2}$, which can not gap out because particle-hole conjugation acts within two of the angular momentum sectors (see the discussion in Sec. III). For the weak phases, this follows because the underlying $C_{2}$-symmetric one-dimensional building blocks have the representation $\Theta=B$, which does not allow for a topological phase. We thus conclude that

$$
\mathcal{K}_{\mathrm{D}}\left[C_{4},{ }^{1,2} E, d=2\right]=\mathbb{Z} .
$$

\section{Three dimensions, class $\mathrm{D}, C_{2 v}$}

In class $\mathrm{D}$, there are no three-dimensional strong firstorder topological phases without crystalline symmetries, so that no first-order phase is possible. To determine whether a strong phase is possible, with protected gapless Majorana modes along mirror-symmetric hinges, we deform the threedimensional crystal into a "cross" of four two-dimensional planes, as shown schematically in Fig. 21(a) and determine below for each representation $\Theta$, whether a phase with chiral hinge states is possible and whether it is intrinsic or extrinsic (i.e., whether it can be removed with a decoration of the surface). Intrinsic second-order boundary signatures are possible in representations $\Theta=A_{2}, B_{1}, B_{2}$ as we show below. To determine whether a third-order TSC is possible, we note that a protected corner state can exist only for a corner on the intersection of the two mirror planes. We find that pairs of corner states are allowed for the representations $\Theta=A_{2}$, $B_{1}$, and $B_{2}$, but not for $\Theta=A_{1}$. However, the corner state

for $\Theta=A_{2}, B_{1}$, or $B_{2}$ is extrinsic, as they can be removed by the decoration of a pair of hinges in a mirror plane with one-dimensional topological superconductors.

Representation $\Theta=A_{1}$. For the representation $A_{1}$ there are no intrinsic second-order boundary signatures: Although the edges of the planes in the deformed structure of Fig. 21(a) do not allow for pairs of copropagating chiral Majorana modes, one for each mirror parity, such configurations of hinge modes can be removed by decorating the four symmetry-related crystal faces by two-dimensional quantum Hall phases, as shown schematically in Fig. 21(b). Further, all lower-dimensional building blocks that can be used for stacking are trivial, which rules out the existence of weak phases. We conclude that

$$
\mathcal{K}_{\mathrm{D}}\left[C_{2 v}, A_{1}, d=3\right]=0 .
$$

Representation $\Theta=A_{2}$. We note that for each of the planes in the deformed structure of Fig. 21(a), one of the mirror symmetries acts as an onsite symmetry, whereas the other mirror symmetry maps planes on opposite sides of the central "cross" onto each other. For representation $A_{2}$, each of the planes in the deformed structure of Fig. 21(a) allows for chiral Majorana modes in both parity sectors of the onsite mirror symmetry. These Majorana modes turn into hinge modes upon deforming back to the full three-dimensional structure. The perpendicular mirror symmetry imposes the condition that opposite hinges have Majorana modes with the same propagation direction, but with opposite mirror parity. The condition that the center of the cross be gapped imposes the requirement that the net number of chiral modes, weighed with propagation direction, is zero. Hence, there is an extrinsic $\mathbb{Z}^{3}$ classification of $C_{2 v}$-compatible hinge modes. To obtain the intrinsic second-order phases, configurations of hinge modes that differ by a change of surface termination must be divided out. Noting that the four surfaces of a $C_{2 v}$-symmetric crystal admit a "decoration" with quantum Hall phases, which gives a pair of co-propagating opposite-parity hinge modes at each mirror-symmetric hinge [see Fig. 21(b)], we find that a classifying group $\mathbb{Z}^{2}$ of intrinsic second-order phases remains. Generator Hamiltonians for these second-order phases are

$$
\begin{aligned}
H_{(3, \pm)}^{\prime}= & \rho_{0} \tau_{3}\left(3-m-\sum_{i=x, y, z} \cos k_{i}\right) \\
& \pm \rho_{1} \tau_{1} \sin k_{x}+\rho_{3} \tau_{1} \sin k_{y}+\rho_{0} \tau_{2} \sin k_{z}
\end{aligned}
$$

with $\quad 0<m<2$ and representations $U\left(\mathcal{M}_{x}\right)=$ $i \rho_{3} \tau_{0}, U\left(\mathcal{M}_{y}\right)=i \rho_{1} \tau_{0}, \mathcal{P}=\rho_{0} \tau_{1} K$.

The weak phases in this symmetry class can be constructed as stacks of one- or two-dimensional topological phases. We find the following possibilities:

(i) Stack of two-dimensional $C_{2 v}$-symmetric $x y$ planes in the $z$ direction with second-order topology. Such planes have a $\mathbb{Z}_{2}^{2}$ classification (see Appendix B 4). These phases are labeled “ $(2 ; z)$ ” in Fig. 16.

(ii) Stack of two-dimensional " $C_{2 v} \mid C_{s}$ "-symmetric $y z$ or $x z$ planes in the $x$ or $y$ direction, respectively. These planes have a $\mathbb{Z}$ classification, corresponding to a first-order twodimensional topological superconductor with counterpropagating Majorana modes in opposite in-plane mirror eigensectors, as shown in Appendix B 3. These phases are labeled 
"( $(2 ; x)$ " and " $(2 ; y)$ " in Fig. 16. Superconductors with even Chern number in a mirror plane can be adiabatically deformed to Chern insulators with vanishing superconducting correlations.

(iii) Stacks of one-dimensional " $C_{2 v} \mid C_{2 v}$ "-symmetric $z$ lines in the $x$ and $y$ directions. These have a $\mathbb{Z}_{2}$ classification, corresponding to a one-dimensional topological superconductor with a pair of Majorana bound states at each end as shown in Appendix B 1. These phases are labeled " $(1 ; x ; y)$ " in Fig. 16.

(iv) Stacks of one-dimensional " $C_{2 v} \mid C_{s}$ "-symmetric $y$ or $x$ lines in the $x$ and $z$ and in the $y$ and $z$ directions, respectively. These, too, have a $\mathbb{Z}_{2}$ classification, corresponding to a one-dimensional topological superconductor with a pair of Majorana bound states at each end as shown in Appendix B 1 . These phases are labeled " $(1 ; x ; z)$ " and " $(1 ; y ; z)$ ' in Fig. 16.

The complete classifying group is hence

$$
\mathcal{K}_{\mathrm{D}}\left[C_{2 v}, A_{2}, d=3\right]=\mathbb{Z}_{2}^{5} \times \mathbb{Z}^{4} .
$$

Representations $\Theta=B_{1,2}$. The representations $B_{1}$ and $B_{2}$ are related by a rotation. For concreteness, we discuss the $B_{2}$ case in the following. In this phase, only one pair of mirror-symmetric hinges allows for intrinsic chiral Majorana modes, whereas any hinge modes appearing at the other pair of mirror-symmetric hinges can always be removed by adding a suitable decoration to the four mirror-related surfaces, as shown schematically in Fig. 21(b). As a result, for the discussion of second-order phases, the three-dimensional crystal may be deformed to a two-dimensional one with $C_{2 v} \mid C_{s}$ symmetry. As shown in Appendix B 3, there exists a strong twodimensional phase with counterpropagating Majorana edge modes with opposite mirror parity. This phase corresponds to a second-order phase of the three-dimensional crystal. One verifies that this second-order phase is intrinsic since a surface decoration with quantum Hall planes is forbidden by the perpendicular mirror symmetry. A generator for the strong second-order phase is

$$
\begin{aligned}
H_{(3)}^{\prime}(\mathbf{k})= & \mu_{0} \rho_{0} \tau_{3}\left(3-m-\cos k_{x}-\cos k_{y}-\cos k_{z}\right) \\
& +\mu_{3} \rho_{0} \tau_{1} \sin k_{y}+\mu_{0} \rho_{0} \tau_{2} \sin k_{z}+\mu_{2} \rho_{2} \tau_{1} \sin k_{x}
\end{aligned}
$$

with $0<m<2$ and the representations $U\left(\mathcal{M}_{x}\right)=i \mu_{3} \rho_{0} \tau_{0}$, $U\left(\mathcal{M}_{y}\right)=i \mu_{2} \rho_{0} \tau_{0}, \mathcal{P}=\mu_{0} \rho_{0} \tau_{1} K$. Second-order topological superconductors with a pair of counterpropagating Majorana modes within in a mirror plane can be adiabatically deformed to normal-state second-order topological insulators with chiral hinge mode.

The weak phases in this symmetry class can be constructed as stacks of one- or two-dimensional topological phases:

(a) A stack of two-dimensional $x y$ planes with $C_{2 v}$ symmetry in the $z$ direction is not possible since there are no strong phases with $C_{2 v}$ symmetry and $\Theta=B_{2}$ in two dimensions (see Appendix B 4).

(b) A stack of two-dimensional $x z$ planes with " $C_{2 v} \mid C_{s}$ " symmetry in the $y$ direction. These planes have a $\mathbb{Z}_{2}$ classification, corresponding to a second-order topological superconducting phase with a pair of Majorana bound states at a corner bisected by the mirror $\mathcal{M}_{x}$ plane, as shown in Appendix B 3 . These phases are labeled " $(2 ; y)$ " in Fig. 17. (c) A stack of two-dimensional $y z$ planes with " $C_{2 v} \mid C_{s}$ " symmetry in the $x$ directions. These planes have a $\mathbb{Z}$ classification, corresponding to a first-order topological superconducting phase with counterpropagating Majorana modes in opposite in-plane mirror eigensectors, as shown in Appendix B 3. These phases are labeled " $(2 ; x)$ " in Fig. 17. Superconductors with even Chern number in a mirror plane can be adiabatically deformed to normal-state Chern insulators.

(d) A stack of one-dimensional $z$ lines with " $C_{2 v} \mid C_{2 v}$ " symmetry in the $x$ and $y$ directions. These lines have a $\mathbb{Z}_{2}$ classification, corresponding to a one-dimensional topological superconductor with a pair of Majorana bound states at each end, as shown in Appendix B 2. These phases are labeled " $(1 ; x, y)$ " in Fig. 17.

(e) A stack of one-dimensional $x$ lines with " $C_{2 v} \mid C_{s}$ " symmetry in the $y$ and $z$ directions does not give a weak phase since there are no appropriate strong phases in one dimension (see Appendix B 1).

(f) A stack of one-dimensional $y$ lines with " $C_{2 v} \mid C_{s}$ ", symmetry in the $x$ and $z$ directions. These lines have a $\mathbb{Z}_{2}$ classification, corresponding to a one-dimensional topological superconducting phase with a pair of Majorana bound states at each end, as shown in Appendix B 1. These phases are labeled " $(1 ; x, z)$ " in Fig. 17.

The complete classifying group is hence

$$
\mathcal{K}_{\mathrm{D}}\left[C_{2 v}, B_{1,2}, d=3\right]=\mathbb{Z}_{2}^{3} \times \mathbb{Z}^{2} .
$$

\section{Three dimensions, class $\mathrm{D}, C_{4}$}

Three-dimensional, fourfold rotation-symmetric topological superconductors can not have first-order boundary signatures, as there are no first-order topological phases in three dimensions for class D. A strong second-order boundary signature is also forbidden as (i) a rotation-symmetric chiral Majorana mode in a plane perpendicular to the rotation axis can be shrunk to a point and (ii) planes parallel to the rotation axis satisfy a $(x, z) \rightarrow(-x, z)$ symmetry, which forbids a chiral mode.

In the following, we determine whether a third-order boundary signature may exist at the rotation axis.

Representations $\Theta=A$ and $B$. For $\Theta=A$ or $B$ the irreducible representations of fourfold rotation are exchanged under particle-hole conjugation as shown in Table V. Each pair is effectively in class $\mathrm{A}$, forbidding a one-dimensional topological phase with boundary signature along rotation axis. Hence, there are no third-order boundary signatures in these representations. Accounting for weak phases stacked in the $z$ direction (parallel to the rotation axis), we thus find that

$$
\begin{aligned}
\mathcal{K}_{\mathrm{D}}\left[C_{4}, A, d=3\right] & =\mathcal{K}_{\mathrm{D}}\left[C_{4}, B, d=3\right] \\
& =\mathbb{Z}_{2}^{2} \times \mathbb{Z},
\end{aligned}
$$

where we used the classification results for two-dimensional phases obtained in Appendix B 5.

Representations $\Theta={ }^{1} E$ and ${ }^{2} E$. As in the main text, the discussion for the two representations $\Theta={ }^{1} E$ or ${ }^{2} E$ are analogous. We focus on the $\Theta={ }^{1} E$ representation in the following. As shown in Table $\mathrm{V}$, the $j=\frac{1}{2}$ and $\frac{5}{2}$ rotation eigenspaces are invariant under particle-hole conjugation while the $j=\frac{3}{2}, \frac{7}{2}$ eigenspaces are exchanged in this 

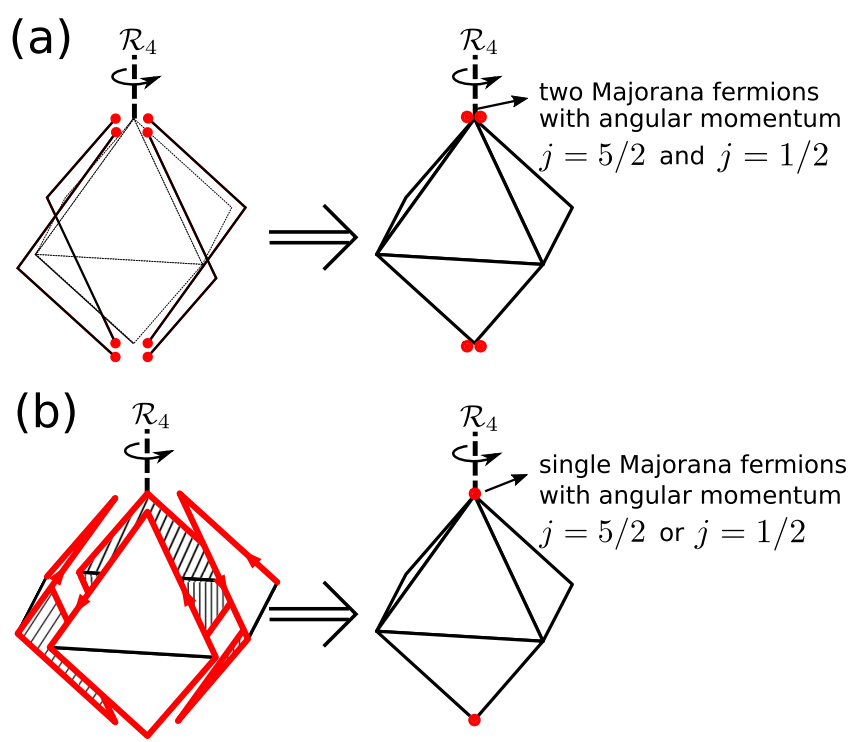

FIG. 22. (a) Decoration consisting of four copies of onedimensional Kitaev chain. This decoration results in two Majorana fermions with angular momentum $j=\frac{1}{2}$ and $\frac{5}{2}$. (b) Decoration consisting of four copies of two-dimensional $p$-wave superconductors, resulting in single Majorana fermion with angular momentum either $j=\frac{1}{2}$ or $\frac{5}{2}$.

symmetry class. The $j=\frac{1}{2}$ and $\frac{5}{2}$ eigenspaces belong to class $\mathrm{D}$ allowing stable zero-dimensional gapless Majorana states. However, below we show that a three-dimensional superconductor with a single Majorana fermion on the fourfold rotation axis is an extrinsic (nonanomalous) third-order phase.

We consider two kinds of decorations [39] (see Fig. 22). The first kind consists of four copies of one-dimensional Kitaev chains placed on the crystal surface and beginning and ending on the rotation axis. The four Kitaev chains are related to each other by a fourfold rotation, which results in four Majorana end states, all with different angular momentum $j$. Majorana end states with $j=\frac{3}{2}$ and $\frac{7}{2}$ are not stable and can be gapped out, while Majorana states with $j=\frac{1}{2}$ and $\frac{5}{2}$ remain. The second type of decoration consists of covering the crystal surface by four copies of a two-dimensional topological superconductor, such that the four two-dimensional superconductors are mapped onto each other by $C_{4}$ symmetry. Majorana modes running along the surface can be gapped out [Fig. 22(b)]. Since $\Theta={ }^{1} E$ or ${ }^{2} E$ representations correspond to an odd-parity superconductor, the resulting $C_{4}$-symmetric spinful superconductor needs to have a vortex at the rotation axis [39]. We therefore conclude that this type of decoration gives rise to a single Majorana bound state at the rotation axis, which needs to have angular momentum either $j=\frac{1}{2}$ or $\frac{5}{2}$. The combination of the two types of decorations described here can account for all configurations of Majorana corner states compatible with the $C_{4}$ rotation symmetry. We conclude that there are no intrinsic third-order anomalous boundary states in this class.

We conclude that the only possible topological phases in three dimensions are weak phases:

(1) A stack of $x y$ planes with $C_{4}$ symmetry in the $z$ direction allows for a Chern superconductor with even Chern number (see Appendix B 5). This phase is labeled " $(2 ; z)$ )" in Fig. 15.

(2) A stack of $x z$ and $y z$ planes related by fourfold rotation symmetry stacked in both $x$ and $y$ directions. As the rotation axis lies in the intersection of the two planes, their classifying group is $\mathcal{K}_{(0,1,0)}\left[C_{s}, A^{\prime \prime}, d=2\right]=\mathbb{Z}_{2}$ allowing a second-order topological superconductor. The configuration has pairs of Majorana bound states at the rotation axis. This phase is labeled " $(2 ; x, y)$ " in Fig. 15.

(3) There is no stack of one-dimensional superconductors pointing in the $x$ and $y$ directions, related by fourfold rotation symmetry, as the corresponding classifying group $\mathcal{K}_{(0,1,0)}\left[C_{s}, A^{\prime \prime}, d=1\right]=0$ is trivial.

(4) A stack of one-dimensional $z$ lines within the rotation axis in the $x$ and $y$ directions. These lines have a classifying group $\mathcal{K}_{(0,1,0)}\left[C_{4},{ }^{1} E, d=1\right]=\mathbb{Z}_{2}^{2}$ corresponding to one-dimensional TSC with Majorana bound states in the class D rotation subspaces $j=\frac{1}{2}, \frac{5}{2}$. The phases are labeled " $(1, j ; x, y)$ " in Fig. 15 .

The complete classifying group is hence

$$
\mathcal{K}_{\mathrm{D}}\left[C_{4},{ }^{1,2} E, d=3\right]=\mathbb{Z}_{2}^{3} \times \mathbb{Z}
$$

\section{APPENDIX C: 1D COMPATIBILITY RELATION FOR CLASS CI WITH INVERSION SYMMETRY}

We here discuss how to obtain the compatibility relation (130) of Sec. VII D. For definiteness, we choose the representations

$$
\mathcal{T}=\rho_{0} \tau_{0} K, \quad \mathcal{P}=\rho_{0} \tau_{2} K, \quad \mathcal{I}=\rho_{0} \tau_{3}
$$

The following combinations of symmetries are local in $\mathbf{k}$ :

$$
\mathcal{I} T=\rho_{0} \tau_{3} K, \quad \mathcal{I} P=\rho_{0} \tau_{1} K
$$

as well as their product, which is a chiral antisymmetry. To construct the compatibility relation (130), we consider the one-parameter family of one-dimensional Hamiltonians $H_{t}(k)$, defined by restricting $H(\mathbf{k})$ to the line $k_{x}=0, k_{y}=t$, $k_{z}=k$. For $t=0$ and $\pi$, the one-dimensional Hamiltonian $H_{t}(k)$ is a one-dimensional Hamiltonian in class CI with inversion symmetry and the $A_{u}$ representation. The topological invariants at high-symmetry momenta for this onedimensional Hamiltonian coincide with the corresponding band labels defined for the full three-dimensional Hamiltonian $H(\mathbf{k})$ and satisfy the same $0 \mathrm{D}$ compatibility relations (see Secs. VII C and VII D). For generic $0<t<\pi, H_{t}(k)$ is a onedimensional Hamiltonian that has the local-in- $k$ symmetries $\mathcal{I} T$ and $\mathcal{I P}$ only. Such a one-parameter family has a strong $\mathbb{Z}_{2}$ invariant [82], which we call $\mathfrak{S}$. We can construct a compatibility relation by relating $\mathfrak{S}$ to the topological band labels of $H_{t}(k)$ at $t=0$ and $\pi$. We find that this can be accomplished without knowledge of a general expression for the strong invariant $\mathfrak{S}$.

Before we can construct such a compatibility relation, it is necessary to obtain the full classification of the inversionsymmetric Hamiltonians $H_{t}(k)$ at $t=0$ and $\pi$, including topologically nontrivial atomic-limit states. We recall that the 
topological band labels for this case are $\left\{\mathfrak{n}_{+}^{(0, t, 0)}, \mathfrak{N}_{+}^{(0, t, \pi)}\right\}$, where

$$
2 \mathfrak{n}_{+}^{(0, t, 0)}=\mathfrak{N}_{+}^{(0, t, 0)}-\mathfrak{N}_{+}^{(0, t, \pi)}, \quad t=0, \pi
$$

(see the discussion in Sec. VII C). It follows that the group of topological band labels is $\mathbb{Z} \times 2 \mathbb{Z}$, where we use $2 \mathbb{Z}$ to indicate the integers spanned by the label $\mathfrak{n}_{+}^{(0, t, \pi)}$. Interpreting the topological band labels in terms of a topological classification, we note that the factor $\mathbb{Z}$ corresponds to weak phases, whereas the factor $2 \mathbb{Z}$ describes strong one-dimensional atomic-limit phases [39]. We use $\left.K_{t}\right|_{t=0, \pi} \simeq 2 \mathbb{Z}$ to denote the strong phases of the model at $t=0$ and $\pi$ and note that the topological band label $2 \mathfrak{n}_{+}^{(0, t, 0)}$ can be used as the corresponding topological invariant.

To obtain the compatibility relation, we have to determine what values of $\mathfrak{n}_{+}^{(0, t, 0)}$ are compatible with a given value of $\mathfrak{S}$. Such a problem requires understanding the homomorphism

$$
\left.\left.K_{t}\right|_{t=0, \pi} \rightarrow K_{t}\right|_{0<t<\pi},
$$

where $\left.K_{t}\right|_{0<t<\pi} \simeq \mathbb{Z}_{2}$ is the group classifying strong phases of $H_{t}(k)$ for generic $t$. To construct this homomorphism, we notice that

$$
H(k)=\rho_{0} \tau_{3}(1-m-\cos k)+\rho_{2} \tau_{1} \sin k
$$

with $0<m<2$ an example of a Hamiltonian that satisfies $\mathcal{T}, \mathcal{P}$, and $\mathcal{I}$ symmetries and that is nontrivial if only the local-in- $k$ symmetries $\mathcal{I} T$ and $\mathcal{I} P$ are kept, i.e., it has invariant $\mathfrak{S}=1$. [Although no general expression for $\mathfrak{S}$ is available, $\mathfrak{S}$ can be calculated as the parity of a winding number if $H(k)$ is a $4 \times 4$ matrix.] It follows that the mapping $\left.K_{t}\right|_{t=0, \pi} \rightarrow$ $\left.K_{t}\right|_{0<t<\pi}$ must be surjective. One verifies that the Hamiltonian (C4) has topological band label $\mathfrak{n}_{+}^{(0)}=1$. Since the trivial phase has $\mathfrak{n}_{+}^{(0)}=0$ and since $\mathfrak{S}$ has a $\mathbb{Z}_{2}$ group structure, it directly follows that

$$
\begin{aligned}
\mathfrak{S} & =\mathfrak{n}_{+}^{(0,0,0)} \bmod 2 \\
& =\mathfrak{n}_{+}^{(0, \pi, 0)} \bmod 2 .
\end{aligned}
$$

This is one of the compatibility relations of Eq. (130). The other relations follow in analogous manner by considering other appropriately chosen families of one-dimensional Hamiltonians $H_{t}(k)$.
[1] K. V. Klitzing, G. Dorda, and M. Pepper, Phys. Rev. Lett. 45, 494 (1980).

[2] A. Y. Kitaev, Phys. Usp. 44, 131 (2001).

[3] D. A. Ivanov, Phys. Rev. Lett. 86, 268 (2001).

[4] C. L. Kane and E. J. Mele, Phys. Rev. Lett. 95, 146802 (2005).

[5] C. L. Kane and E. J. Mele, Phys. Rev. Lett. 95, 226801 (2005).

[6] V. Mourik, K. Zuo, S. M. Frolov, S. R. Plissard, E. P. A. M. Bakkers, and L. P. Kouwenhoven, Science 336, 1003 (2012).

[7] C. Brüne, C. X. Liu, E. G. Novik, E. M. Hankiewicz, H. Buhmann, Y. L. Chen, X. L. Qi, Z. X. Shen, S. C. Zhang, and L. W. Molenkamp, Phys. Rev. Lett. 106, 126803 (2011).

[8] A. Altland and M. R. Zirnbauer, Phys. Rev. B 55, 1142 (1997).

[9] A. Kitaev, in Advances in Theoretical Physics: Landau Memorial Conference, AIP Conference Proceedings, Vol. 1134 (AIP, Melville, NY, 2009), p. 22.

[10] A. P. Schnyder, S. Ryu, A. Furusaki, and A. W. W. Ludwig, in Advances in Theoretical Physics: Landau Memorial Conference, AIP Conference Proceedings, Vol. 1134 (AIP, Melville, NY, 2009), p. 10.

[11] L. Fu, Phys. Rev. Lett. 106, 106802 (2011).

[12] S. A. Parameswaran and Y. Wan, Physics 10, 132 (2017).

[13] F. Schindler, A. M. Cook, M. G. Vergniory, Z. Wang, S. S. P. Parkin, B. A. Bernevig, and T. Neupert, Sci. Adv. 4, eaat0346 (2018)

[14] Y. Peng, Y. Bao, and F. von Oppen, Phys. Rev. B 95, 235143 (2017)

[15] J. Langbehn, Y. Peng, L. Trifunovic, F. von Oppen, and P. W. Brouwer, Phys. Rev. Lett. 119, 246401 (2017).

[16] Z. Song, Z. Fang, and C. Fang, Phys. Rev. Lett. 119, 246402 (2017)

[17] C. Fang and L. Fu, Sci. Adv. 5, eaat2374 (2019).

[18] M. Ezawa, Phys. Rev. Lett. 120, 026801 (2018).
[19] H. Shapourian, Y. Wang, and S. Ryu, Phys. Rev. B 97, 094508 (2018).

[20] X. Zhu, Phys. Rev. B 97, 205134 (2018).

[21] Z. Yan, F. Song, and Z. Wang, Phys. Rev. Lett. 121, 096803 (2018).

[22] Y. Wang, M. Lin, and T. L. Hughes, Phys. Rev. B 98, 165144 (2018).

[23] Q. Wang, C.-C. Liu, Y.-M. Lu, and F. Zhang, Phys. Rev. Lett. 121, 186801 (2018).

[24] E. Khalaf, H. C. Po, A. Vishwanath, and H. Watanabe, Phys. Rev. X 8, 031070 (2018).

[25] E. Khalaf, Phys. Rev. B 97, 205136 (2018).

[26] N. Okuma, M. Sato, and K. Shiozaki, Phys. Rev. B 99, 085127 (2019).

[27] H. C. Po, A. Vishwanath, and H. Watanabe, Nat. Commun. 8 , 50 (2017).

[28] B. Bradlyn, L. Elcoro, J. Cano, M. G. Vergniory, Z. Wang, C. Felser, M. I. Aroyo, and B. A. Bernevig, Nature (London) 547, 298 (2017).

[29] A. M. Turner, Y. Zhang, R. S. K. Mong, and A. Vishwanath, Phys. Rev. B 85, 165120 (2012).

[30] A. Lau, C. Ortix, and J. van den Brink, Phys. Rev. B 91, 085106 (2015).

[31] A. Lau, J. van den Brink, and C. Ortix, Phys. Rev. B 94, 165164 (2016).

[32] W. A. Benalcazar, B. A. Bernevig, and T. L. Hughes, Science 357, 61 (2017).

[33] W. A. Benalcazar, B. A. Bernevig, and T. L. Hughes, Phys. Rev. B 96, 245115 (2017).

[34] G. van Miert and C. Ortix, Phys. Rev. B 96, 235130 (2017).

[35] J.-W. Rhim, J. Behrends, and J. H. Bardarson, Phys. Rev. B 95, 035421 (2017).

[36] K. Shiozaki and M. Sato, Phys. Rev. B 90, 165114 (2014). 
[37] K. Shiozaki, M. Sato, and K. Gomi, Phys. Rev. B 93, 195413 (2016).

[38] C.-K. Chiu, J. C. Y. Teo, A. P. Schnyder, and S. Ryu, Rev. Mod. Phys. 88, 035005 (2016).

[39] L. Trifunovic and P. W. Brouwer, Phys. Rev. X 9, 011012 (2019).

[40] E. Cornfeld and A. Chapman, Phys. Rev. B 99, 075105 (2019).

[41] K. Shiozaki, arXiv:1907.09354.

[42] S.-J. Huang, H. Song, Y.-P. Huang, and M. Hermele, Phys. Rev. B 96, 205106 (2017).

[43] R. Thorngren and D. V. Else, Phys. Rev. X 8, 011040 (2018).

[44] C. Fang, M. J. Gilbert, and B. A. Bernevig, Phys. Rev. B 86, $115112(2012)$

[45] C. Fang, M. J. Gilbert, and B. A. Bernevig, Phys. Rev. B 87, 035119 (2013).

[46] C.-K. Chiu, H. Yao, and S. Ryu, Phys. Rev. B 88, 075142 (2013).

[47] P. Jadaun, D. Xiao, Q. Niu, and S. K. Banerjee, Phys. Rev. B 88, 085110 (2013).

[48] T. Morimoto and A. Furusaki, Phys. Rev. B 88, 125129 (2013).

[49] J. C. Y. Teo and T. L. Hughes, Phys. Rev. Lett. 111, 047006 (2013).

[50] W. A. Benalcazar, J. C. Y. Teo, and T. L. Hughes, Phys. Rev. B 89, 224503 (2014).

[51] X.-J. Liu, J. J. He, and K. T. Law, Phys. Rev. B 90, 235141 (2014).

[52] A. Alexandradinata, C. Fang, M. J. Gilbert, and B. A. Bernevig, Phys. Rev. Lett. 113, 116403 (2014).

[53] F. Zhang, C. L. Kane, and E. J. Mele, Phys. Rev. Lett. 111, 056403 (2013).

[54] R.-J. Slager, A. Mesaros, V. Juricic, and J. Zaanen, Nat. Phys. 9, 98 (2013).

[55] X.-Y. Dong and C.-X. Liu, Phys. Rev. B 93, 045429 (2016).

[56] J. Kruthoff, J. de Boer, J. van Wezel, C. L. Kane, and R.-J. Slager, Phys. Rev. X 7, 041069 (2017).

[57] L. Fu and C. L. Kane, Phys. Rev. B 76, 045302 (2007).

[58] Z. Song, T. Zhang, and C. Fang, Phys. Rev. X 8, 031069 (2018).

[59] Z. Song, T. Zhang, and C. Fang, Nat. Commun. 9, 3530 (2018).

[60] T. Zhang, Y. Jiang, Z. Song, H. Huang, Y. He, Z. Fang, H. Weng, and C. Fang, Nature (London) 566, 475 (2019).
[61] L. P. Gorkov, ZhETF 34, 735 (1958) [Sov.-Phys. JETP 7, 505 (1958)].

[62] P. G. de Gennes, Superconductivity of Metals and Alloys (Benjamin, New York, 1966).

[63] S. Ono and H. Watanabe, Phys. Rev. B 98, 115150 (2018).

[64] K. Shiozaki, M. Sato, and K. Gomi, arXiv:1802.06694.

[65] S. Ono, Y. Yanase, and H. Watanabe, Phys. Rev. Research 1, 013012 (2019).

[66] A. Skurativska, T. Neupert, and M. H. Fischer, Phys. Rev. Res. 2, 013064 (2020).

[67] S. Ono, H. C. Po, and H. Watanabe, Sci. Adv. 6, eaaz8367 (2020).

[68] K. Shiozaki, arXiv:1907.13632.

[69] M. Sigrist and K. Ueda, Rev. Mod. Phys. 63, 239 (1991).

[70] M. Geier, L. Trifunovic, M. Hoskam, and P. W. Brouwer, Phys. Rev. B 97, 205135 (2018).

[71] C. J. Bradley, The Mathematical Theory of Symmetry in Solids: Representation Theory for Point Groups and Space Groups (Clarendon, Oxford, 1972).

[72] S. Ryu, A. P. Schnyder, A. Furusaki, and A. W. W. Ludwig, New J. Phys. 12, 065010 (2010).

[73] M. Nakahara, Geometry, Topology and Physics, 2nd ed., Graduate Student Series in Physics (Taylor \& Francis, New York, 2003).

[74] M. S. Dresselhaus, G. Dresselhaus, and A. Jorio, Group Theory: Application to the Physics of Condensed Matter (Springer, Berlin, 2007).

[75] C. Fang, Y. Chen, H.-Y. Kee, and L. Fu, Phys. Rev. B 92, 081201(R) (2015).

[76] J. Ahn, D. Kim, Y. Kim, and B.-J. Yang, Phys. Rev. Lett. 121, 106403 (2018).

[77] M. Sato, Phys. Rev. B 81, 220504(R) (2010).

[78] L. Fu and E. Berg, Phys. Rev. Lett. 105, 097001 (2010).

[79] See Supplemental Material at http://link.aps.org/supplemental/ 10.1103/PhysRevB.101.245128 for the tabulated topological band labels of all generators of atomic limits and topological crystalline phases of all examples contained in the article.

[80] L. Trifunovic and P. W. Brouwer, Phys. Rev. B 96, 195109 (2017).

[81] N. Bultinck, B. A. Bernevig, and M. P. Zaletel, Phys. Rev. B 99, 125149 (2019).

[82] J. C. Y. Teo and C. L. Kane, Phys. Rev. B 82, 115120 (2010).

[83] C. W. Groth, M. Wimmer, A. R. Akhmerov, and X. Waintal, New J. Phys. 16, 063065 (2014). 